\title{
Macroscopic Modeling of Polymer-Electrolyte Membranes
}

\author{
Adam Z. Weber and John Newman \\ Lawrence Berkeley National Laboratory and \\ Department of Chemical Engineering, \\ University of California \\ Berkeley, CA 94720-1462
}

\begin{abstract}
In this chapter, the various approaches for the macroscopic modeling of transport phenomena in polymer-electrolyte membranes are discussed. This includes general background and modeling methodologies, as well as exploration of the governing equations and some membranerelated topic of interest.
\end{abstract}

*Corresponding author: azweber@lbl.gov; phone: 510-643-1972; fax: 510-642-4778 


\section{Contents}

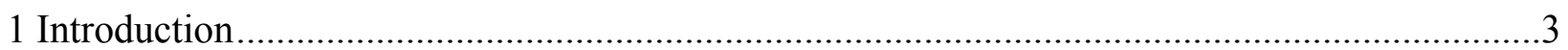

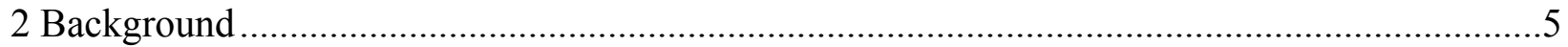

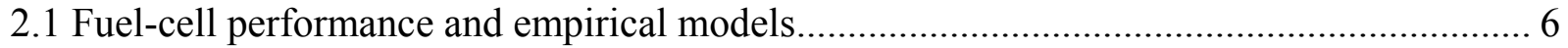

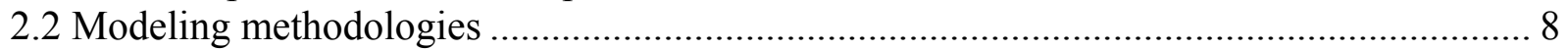

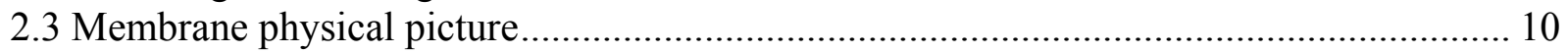

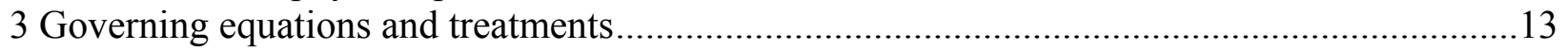

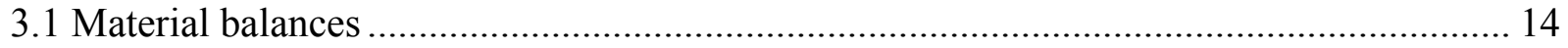

3.2 Proton and water transport phenomena ……………............................................... 15

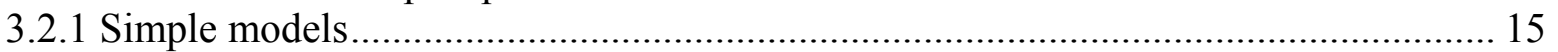

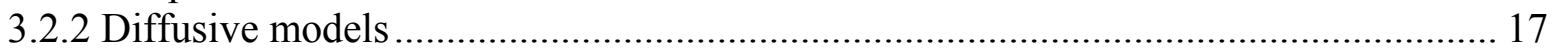

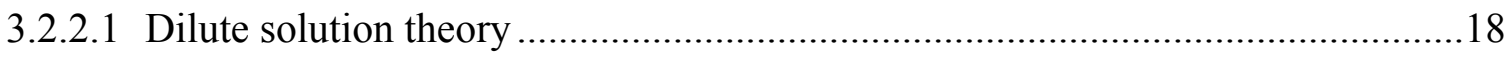

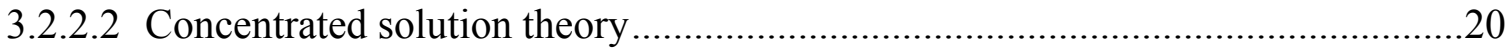

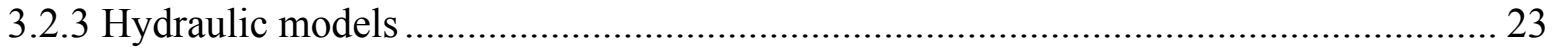

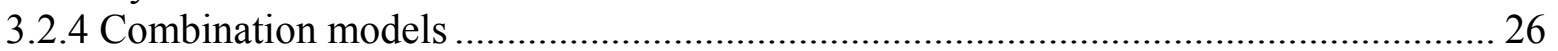

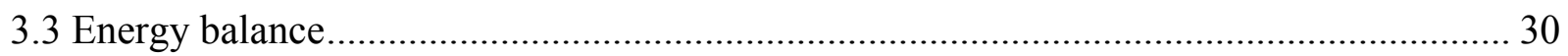

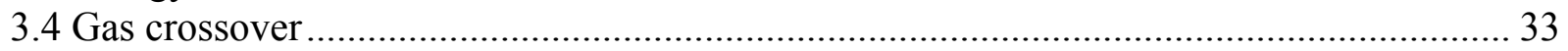

3.5 Boundary conditions and solution methods.................................................................. 36

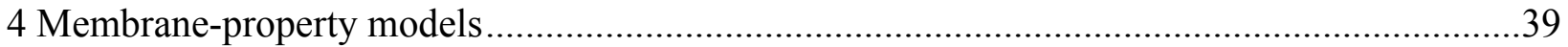

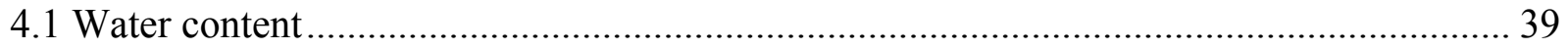

4.1.1 Vapor- to liquid-equilibrated transition (Schröder's paradox) ………………............ 47

4.2 Membrane swelling (thickness) .................................................................................. 50

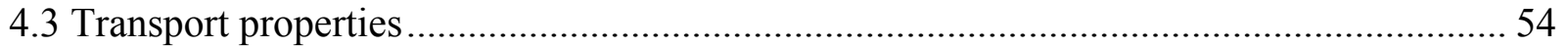

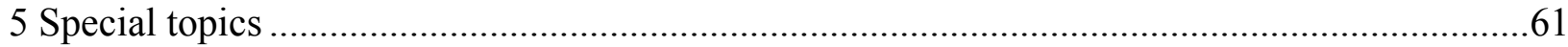

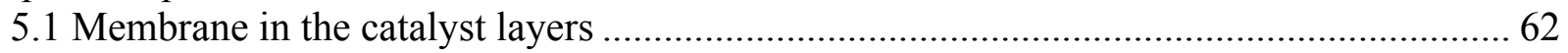

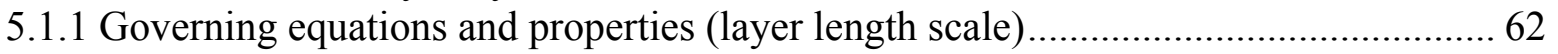

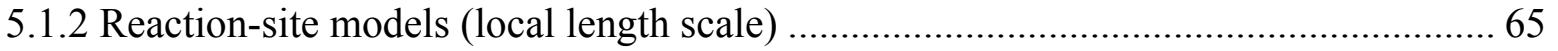

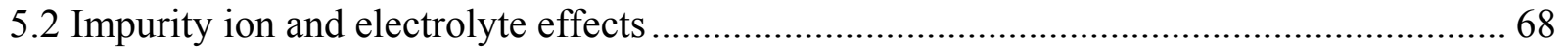

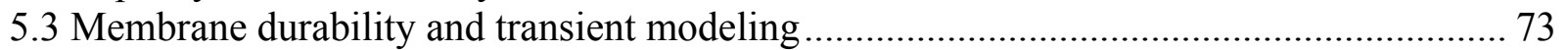

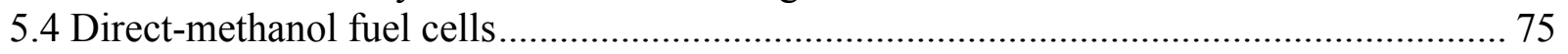

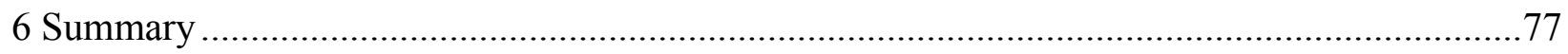

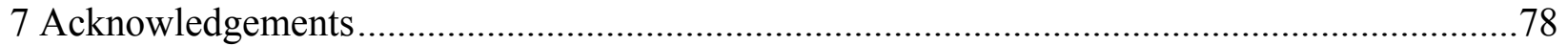

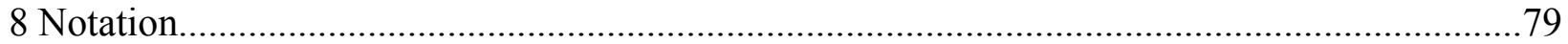

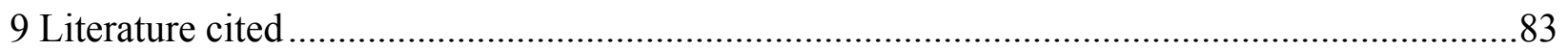




\section{Introduction}

The polymer-electrolyte or proton-exchange membrane (PEM) is the heart of the so-called fuel-cell sandwich (i.e., membrane, catalyst layers, and diffusion media), and hence, the entire fuel cell. It is this electrolyte that makes polymer-electrolyte fuel cells (PEFCs) unique. Correspondingly, the electrolyte must have very specific properties; it needs to conduct protons but not electrons, as well as inhibit gas transport in the separator but allow it in the catalyst layers. Furthermore, the membrane is one of the most important items in the crucial topic of PEFC water management. It is for these reasons as well as others that modeling and experiments of the membrane have been pursued more than any other layer [1].

Although there have been various membranes used, none is more researched or seen as the standard than the Nafion ${ }^{\circledR}$ family by E. I. du Pont de Nemours and Company. Like almost all fuel-cell membranes, Nafion ${ }^{\circledR}$ is a copolymer with essentially hydrophobic and hydrophilic moieties. Specifically, Nafion ${ }^{\circledR}$ is a copolymer of polytetrafluoroethylene and polysulfonyl fluoride vinyl ether; its formula is given in Figure 1. These perfluorinated sulfonic acid (PFSA) ionomers have fixed anions, which are sulfonic acid sites, and consequently, by electroneutrality, the concentration of positive ions remains fixed. They also exhibit many interesting properties such as a high conductivity, prodigious water uptake, and high anion exclusion, to name a few. Nafion ${ }^{\circledR}$ is the main membrane discussed in this review.

Although Nafion ${ }^{\circledR}$ is the focus, the models presented can (and some have been) easily adapted for other membranes such as hydrocarbon ones. Such an adaptation requires altering the various membrane physical and transport properties, but not the governing equations (i.e., the same underlying physics govern transport of the various species). The main reason is that the models presented herein are macroscopic and basically average over the microscale 
heterogeneities that make the membranes unique. In a similar fashion, although the models reviewed and discussed are primarily for hydrogen-fueled PEFCs, they can be used and appropriately altered for other systems like direct-methanol fuel cells (DMFCs).

The focus of this review is to discuss the different macroscopic fuel-cell-membrane models with the overall goal of presenting a picture of the various types of transport in the membrane. Although the majority of the relevant literature models has been examined, there are undoubtedly some that were left out. This is especially the case for those models that have a membrane model but it is not the important feature. For such cases, reference is made to a representative example model from the research group, even though there may be multiple papers published from that group using that model but focusing on other PEFC layers and phenomena. In terms of time frame, this review focuses on models that have been published through the middle of 2005.

There are four other recent review articles dealing with membrane modeling that should be noted. First, those of Weber and Newman [1] and Wang [2] examined macroscopic fuel-cell models through the end of 2004, but only briefly touched on the membrane models. Similarly, that of Fimrite et al. [3] did not go into a lot of depth in terms of membrane models, and it focused mainly on those that use concentrated solution theory. Finally, the review of Kreuer et $a l$. [4] examined microscopic and atomistic membrane models in great detail, and thus they are not addressed in this chapter.

This review chapter can be broken down into various sections. First, background is presented discussing modeling methodologies and a physically based, qualitative model of the structure of the membrane. Next, the general governing equations are discussed in terms of the various modeling approaches, including proton, water, energy, and gas fluxes. The third main section deals with models aimed at predicting transport properties such as conductivity and 
especially the membrane water content. In the final main section, some specialized topics in membrane modeling are mentioned including membrane in the catalyst layers, effect of ions in the membrane, durability, and DMFCs.

\section{Background}

At its simplest, a membrane model should predict the water flux and the potential drop through the membrane. The potential drop is necessary in calculating polarization effects, and the water flux directly impacts the water management of the fuel cell, which is perhaps the most significant component of fuel-cell performance. The models used to predict these two quantities vary greatly in complexity. While some try to understand fundamentally what is occurring in the membrane, others just calculate the values and perhaps only empirically. While the former are useful in optimizing and designing membranes, the latter are favored in complex simulations such as those examining stack or three-dimensional (3-D) effects [1]. Most of the models discussed in this chapter focus on the more complex and macroscopic approaches.

A good model of the membrane must contain certain key elements. Foremost among these is that it must be based on and agree with the physical reality and phenomena that have been observed with these membranes. Furthermore, expressions for the various properties of the membrane should have the relevant dependences such as on temperature and water content. These property expressions can be found by everything from empirical relations to detailed molecular modeling. The water content should also be modeled or accounted for and allowed to vary in a systematic and continuous fashion. Finally, a model should describe the three main fluxes in the membrane, as shown in Figure 2. 
The three main fluxes through the membrane are a proton flux that goes from anode to cathode, a water electro-osmotic flux that develops along with the proton flux, and a watergradient flux. This last flux is sometimes known as the water back flux or back-diffusion flux, and, as discussed in section 3.2, has various interpretations including diffusion, convection, and combinations thereof. Furthermore, these interpretations often serve to differentiate membrane models. In addition to the above three fluxes, there are also fluxes due to crossover of oxygen and hydrogen, which are described in section 3.4.

This section is comprised of two main themes that set the stage for the presentation and discussion of the various models in later sections. The first theme is the modeling methodology, and the second is the physical model or representation of membrane structure as a function of water content. Before proceeding to discuss these themes, some mention should be made about empirical models and fuel-cell performance in general.

\subsection{Fuel-cell performance and empirical models}

As noted above, a membrane model is mainly about predicting performance issues due to transport in the membrane. The performance of a fuel cell is most often reported in the form of a polarization curve, as shown in Figure 3. Roughly speaking, the polarization curve can be broken down into three main regions. At low currents, the behavior of a fuel cell is dominated by kinetic losses, which are not directly attributable to the membrane. As the current is increased, ohmic losses become a factor in lowering the overall cell potential. These ohmic losses are mainly from ionic losses in the membrane both in the separator and the electrodes. At high currents, mass-transport limitations become increasingly important. These losses are due to reactants not being able to reach the reaction sites. Typically, oxygen is the problem due to 
flooding of the cathode by liquid water, and thus the water management role that the membrane plays is important. Of greater impact by the membrane is that if it becomes dehydrated, a limiting current can be realized due to the protons inability to reach the reactive sites.

To capture the above effects, including those in the membrane, empirical models have been used. These are not rigorous models, per se, but instead are educated curve fits using at most semiempirical expression $[1,5,6]$; an example of such a curve is $[1,7,8]$

$$
V=U^{\theta}-b \log (i)-R_{\Omega} i+b \log \left(1-\frac{i}{i_{\lim }}\right)
$$

where $R_{\Omega}$ is the constant resistance in the fuel cell, $b$ is the Tafel slope, $U^{\theta}$ is the standard cell potential, and $V, i$, and $i_{\lim }$ are the cell potential, current density, and limiting current density, respectively. While such an approach is useful in modeling complex geometries and stacks, it is not predictive and not truly a model of the membrane. This is especially apparent in examining Figure 3, where one cannot easily distinguish the three regions from one another just by looking at the polarization curve (solid line). For example, the so-called ohmic or linear portion of the curve actually has mass-transport and other effects in it, as seen in the breakdown of the curve in the figure. Thus, a fit to a polarization curve is not truly yielding the resistance of the membrane, which may not even have a uniform conductivity. Furthermore, the impact of the role that the membrane plays in water management cannot be quantified using such a simple approach.

To expand on the last point, empirical models also may or may not examine the water balance, and if they do, then a net water flux through the membrane is used. This value is either calculated from experimental water-balance data or just assumed. The latter is typically used when the goal of the model is to examine some other fuel-cell layer such as the diffusion media or catalyst layers. The assumption of a net water flux greatly aids in the convergence and 
robustness of a program; however, like using a resistance above, it makes the model less predictive and helpful in understanding membrane phenomena.

\subsection{Modeling methodologies}

In terms of both quantitative and qualitative modeling, PEMs have been modeled within two extremes, the macroscopic and the microscopic, as discussed in recent review articles [1, 2, 4]. The microscopic models provide the fundamental understanding of processes like diffusion and conduction in the membrane on a single-pore or even molecular level. They allow for the evaluation of how small perturbations like heterogeneity of pores and electric fields affect transport, as well as the incorporation of small-scale effects. Although the microscopic models may provide more realistic conditions and factors, they require a lot more knowledge of the microstructure and are much more computationally expensive. For these reasons and also to allow modeling of entire fuel-cell behavior, macroscopic models are more commonly used, although some microscopic details should be incorporated into them. This review focuses on macroscopic models or those that utilize a macrohomogeneous approach.

In a macrohomogeneous approach, the exact geometric details of the modeling domain are neglected. Instead, the domain is treated as a randomly arranged structure that can be described by a small number of variables. Furthermore, transport properties within the domain are averaged over the membrane volume. Thus, all variables are defined at all positions within the domain. Averaging is performed over a region that is small compared to the size of the membrane, but large compared to its microstructure.

The macroscopic membrane models can be placed into three main categories. The differentiation is basically made on how the models treat water movement in the membrane. 
This distinction is also typically what differentiates fuel-cell models from each other. The first category treats water transport as if by diffusion, and thus is termed diffusive. This type of model implicitly assumes that the membrane is a single phase and was popularized for fuel cells by Fuller and Newman [9] and Springer et al. [10] A problem with this approach is that when the membrane is saturated, it does not make sense to have only a diffusive flow since the concentration of water in the membrane is uniform; there is no concentration gradient. Hence, another model should be used in this case (e.g., a hydraulic one).

The second category of membrane models treats the water movement as if by convection, and thus is termed hydraulic. This system explicitly treats the membrane as two phases. The most recognized type of this model was done by Bernardi and Verbrugge [11, 12]. A problem with these models arises for the case of a membrane in a low-relative-humidity reservoir. In such a system there is not a continuous liquid pathway across the medium, and the membrane matrix interacts significantly with the water due to the binding and solvating of the sulfonic acid sites. Thus, a concentration gradient and not one of hydraulic pressure of liquid water, which might not even be defined, seems to be the more appropriate driving force; a one-phase model should be used.

The third category treats the water movement as if by a combination of the above two methods, and thus is termed combination. Such models aim to explain transport across the whole range of possible membrane water contents and bridge the gap between the two categories above. Essentially, the two approaches above operate at one limit of water concentration, and are then somehow averaged between those limits $[13,14]$. The three main model categories are examined in more detail and in terms of equations in section 3.2 


\subsection{Membrane physical picture}

To model the membrane, one requires a physical basis or picture in order to ensure the correct mathematical treatment and application of the underlying physics and phenomena. The crucial component for such a picture is how the membrane changes and interacts with water as a function of water content.

The water content of a membrane is typically given in terms of $\lambda$, a ratio of the moles of water per mole of sulfonic acid sites. This value is normally given in the form of a water-uptake isotherm [15-20], as shown in Figure 4. Such an isotherm is typically determined experimentally, but there are some models that aim to predict at least part of the curve as discussed in section 4.1. The dashed line in Figure 4 represents what is known as Schröder's paradox [21], a phenomenon which occurs in many different polymers and gels. In essence, the paradox is that the membrane exhibits a difference in water uptake (and therefore other properties) only due to the phase of the reservoir in contact with the membrane (i.e., the chemical potential remains constant). As seen in Figure 4, the water content of the membrane in a saturated-vapor reservoir is significantly lower than that in a liquid-water reservoir. This is an important issue since fuel cells are often operated with humidified gases, resulting in situations where there is liquid water on the cathodic side of the membrane and only water vapor on the anodic side. With this introduction, one can now dissect the isotherm and relate it to the membrane microstructure.

The general structure of Nafion ${ }^{\circledR}$, and ionomers in general, as a function of water content has been the source of many studies, as recently reviewed by Mauritz and Moore [22] and Kreuer et al. [4]. The experimental data show that a hydrated membrane phase segregates into ionic and matrix or nonionic phases. The ionic phase is associated with the hydrated sulfonic acid groups, 
and the matrix phase with the polymer backbone. Thus, water is associated with the hydrophilic ionic phase and not the hydrophobic matrix phase. The actual way in which the phases segregate within the polymer depends on the water content and other factors including pretreatment procedures, operating temperature, side-chain length, and equivalent weight, to name a few. Finally, based on various experimental data, all species transported through the membrane move by way of the ionic phase $[10,23,24]$. Therefore, the nonionic fluorocarbon matrix can be taken as inert. Its roles are to add mechanical strength and hydrophobicity, thus aiding in the membrane microstructure and preventing dissolution.

Starting from the dry-membrane case, water initially hydrates the sulfonic acid sites and allows them to dissociate partially. This first water is very tightly bound and hard to move. The next few water molecules serve to hydrate the sulfonic acid sites further beyond their primary shell. This allows for a reorganization of the membrane microstructure into ionic and nonionic domains. Consequently, the conductivity of the membrane greatly increases when this occurs $(\lambda \approx 2)[25]$. As the membrane continues to take up water, the ionic domains continue to organize and enlarge. The ideal picture of this phase segregation is known as the cluster-network model first proposed by Hsu and Gierke [24]. In this idealized picture, the water is contained in a spherical domain about $4 \mathrm{~nm}$ in diameter into which the polymer side-chains infiltrate. The inverse micelle domains form based on a balance between the surface or electrostatic energy due to the coulombic repulsions of the sulfonic acid groups and the elastic or deformation energy due to the work required to deform the polymer matrix. The ionic clusters are connected by interfacial regions or bridging-site pathways about $1 \mathrm{~nm}$ in diameter [26]. These were determined by Hsu and Gierke to be transient connections with a stability on the order of ambient thermal fluctuations, in agreement with molecular-dynamics simulations $[4,27,28]$. 
While the cluster-network model is an idealized picture, it provides a useful visualization of the polymer phase-separated microstructure.

As the membrane becomes more hydrated, the sulfonic acid sites become associated with more water, allowing for a less bound and more bulk-like water to form. This new water is no longer strongly influence by the dielectric properties of the sulfonic acid groups and is essentially enlarging the ionic domains by filling them in with water. This is why there is a flattening out of the slope above $\lambda=6$ in the uptake isotherm (see Figure 3). The extreme case is when the membrane is placed in a liquid-water reservoir, where the ionic domains swell and a bulk-like liquid-water phase comes into existence throughout the membrane. The way in which this rearrangement and phase-transition-type behavior occurs is currently unknown exactly. However, it is probably due to the interfacial properties of the membrane, such as the fluorocarbon-rich skin on the surface of $\operatorname{Nafion}^{\circledR}[29,30]$ or the removal of a liquid-vapor meniscus at the membrane surface [31]. Overall, the final picture of a liquid-equilibrated membrane is a porous structure, with average channel and cluster sizes between 1 and $2 \mathrm{~nm}$ and 2 to $4 \mathrm{~nm}$, respectively [32,33].

In summary, Figure 5 is a schematic of how water interacts with the membrane. In the first panel, at low water contents, the water is strongly bound and solvates the sulfonic acid groups. Additional water causes the water to become less bound, with some bulk-like water forming, the second panel. With more water uptake, ionic domains grow and form interconnections with each other through a percolation-type phenomenon. The connections, or collapsed channels, are transitory and have low concentrations of sulfonic acid sites (similar to the first two panels). The lower left panel corresponds to a membrane that is in contact with saturated water vapor, where such a cluster-channel network has formed. When there is liquid water at the boundary of the 
membrane, structural reorganization and a phase transition occur, allowing for bulk-like liquid water to exist in the channels, resulting in a pore-like structure, the final panel in Figure 5. Because the channels are now filled with liquid, the uptake of the membrane has increased without a change in the chemical potential of the water (i.e., Schröder's paradox). Finally, it was

noted above that the cluster-channel representation is very idealized. A more realistic representation based on experimental data is represented in Figure 6. In the figure, clusters and channels can still be identified, but the clusters are not spherical. Instead, they are more like pore-junction regions where channels cross.

\section{Governing equations and treatments}

One needs the same number of equations as unknowns. Typically, this requires four equations for the four unknowns in the membrane of water flux, water chemical potential, electrical potential, and current density. As mentioned above, the main difference between membrane models basically comes down to how they treat transport, and specifically water transport, in the membrane. While some models may use driving forces of pressure or concentration, depending on what is assumed to be dominating, the correct one to use is chemical potential. As will be shown, this driving force is thermodynamically rigorous as it incorporates the other driving forces.

Besides the transport equations, there are also the other governing equations, most of which are the same for the different models. The membrane modeling equations can be broken down into four main types. The first are the conservations equations, the second are the transport relations, the third are equilibrium relationships, and the fourth are the auxiliary or supporting relations, which include variable definitions, property expressions, and such relations as 
Faraday's law. In this section, the various equations are examined. The discussion is divided based on the phenomenon that the governing equation addresses. Furthermore, the auxiliary equations and equilibrium relationships may depend on the modeling approach and equations, or may even be separate models (see section 4).

\subsection{Material balances}

The conservation of material equations are essentially identical for the various membrane models. Furthermore, they are also relatively simple since there is nothing occurring in the membrane in terms of reactions or source terms. It is necessary to write a material balance for each independent component in the membrane. In differential form, this can be expressed as

$$
\frac{\partial c_{i}}{\partial t}=-\nabla \cdot \mathbf{N}_{i}
$$

where $c_{i}$ and $\mathbf{N}_{i}$ are the concentration and flux density of species $i$, respectively.

The term on the left side of the equation is the accumulation term, which accounts for the change in the total amount of species $i$ held in the membrane within a differential control volume. This term is assumed to be zero for almost all of the membrane models discussed in this review because they are at steady state (except for those discussed in section 5.3). The term on the right side of the equation keeps track of the material that enters or leaves the control volume by mass transport.

Because protons are the only mobile ionic species in the membrane, the proton flux and current density can be related through Faraday's

$$
\mathbf{N}_{\mathrm{H}^{+}}=\frac{\mathbf{i}}{F}
$$


where $\mathbf{i}$ is the superficial current density in the membrane and $F$ is Faraday's constant. If one assumes electroneutrality,

$$
\sum_{i} z_{i} c_{i}=0
$$

where $z_{i}$ is the charge number of species $i$, then by conservation of charge and substitution of equation 3 into equation 2 results in the governing equation for the current (or proton flux) of

$$
\nabla \cdot \mathbf{i}=0
$$

The assumption of electroneutrality implies that the diffuse double layer, where there is significant charge separation, is small compared to the volume of the domain, which is normally the case (for exceptions see section 5.2). Also, double-layer charging is ignored in the above equation since double layers are only expected to exist at the membrane interfaces and not inside the membrane.

\subsection{Proton and water transport phenomena}

The major species being transported in the membrane are water and protons, since the anionic sulfonic acid sites are tethered to the membrane backbone. Thus, two transport equations are required. As discussed in section 2.2, there are three main categories of models, the diffusive, the hydraulic, and the combination. Each is discussed below in turn, but before discussing them, some mention should be made about simple approaches.

\subsubsection{Simple models}

The simplest models of the membrane that treat transport in the membrane in a nontrivial manner (i.e., they consider species transport and not just fit a polarization curve with empirical parameters) make several assumptions and use some simple transport expressions along with the 
material conservation equations above. These models are aimed at examining effects outside the membrane (e.g., cathode flooding [34-48]) or when only general trends are desired [49-54]. Furthermore, these models assume constant values of transport properties in the membrane and thus are not applicable when the water content of the membrane is expected to vary (e.g., membrane dehydration). Such assumptions also limit the predictability of these models.

Since the membrane is stationary, only the water and protons move in the membrane system. For the proton movement, the simplest treatment is to use Ohm's law (equation 29 in differential form)

$$
\mathbf{i}=-\kappa \nabla \Phi
$$

where $\kappa$ is the ionic conductivity of the membrane (which is assumed uniform) and $\Phi$ is the electrical potential in the membrane, which is defined with reference to a standard hydrogen electrode at the same temperature and electrolyte (e.g., Nafion $\left.{ }^{\circledR}\right)$ as the solution of interest (i.e., it carries its own extraneous phases with it). The above expression can easily be integrated to yield a resistance for use in a polarization equation (see section 2.1).

For the movement of water through the membrane, a value of $\beta$, the net water flux per proton flux,

$$
\beta=\frac{\mathbf{N}_{\mathrm{w}}}{\mathbf{i} / F}
$$

is often assumed or is calculated based on the water flux in the anode or cathode region. Most models that use this approach also treat the catalyst layer as an interface, which allows for the water flux to become a boundary condition that is directly related to $\beta$. While this approach has merit in terms of convergence issues and allowing for analytic expressions to describe the membrane, it has limited usage under most fuel-cell conditions since $\beta$ is not known a priori. 
Before proceeding to discuss more rigorous treatments of transport in the membrane, two mentions should be made about hybrid approaches. In the first approach [55], the transport equations are solved in a more rigorous fashion as discussed below, and a database generated of values of $\beta$ and $\mathbf{i}$ as a function of the ratio of $\lambda$ at the anode and cathode and a dimensionless Peclet number for water in the membrane. Such a database is useful for complicated and computationally costly simulations (e.g., 3-D computational-fluid-dynamics ones). In the second hybrid approach [56-58], the conductivity is modeled rigorously, and the transport of water is basically ignored. The reason for this is that the models are more concerned with examining the polarization effects of the cell and not the water management. While this approach is all right for optimization, very complex models, or effects outside of the membrane, ignoring fuel-cell watermanagement is not generally advisable.

\subsubsection{Diffusive models}

The diffusive models treat the membrane system as a single, homogeneous phase in which water and protons dissolve and move by concentration and potential gradients. They correspond more-or-less to the vapor-equilibrated membrane (see Figure 5), or in other words a membrane at lower water content. Many membrane models, including some of the earliest ones, treat the system in such a manner. The diffusive models allow one to predict such effects as membrane dehydration, as shown in Figure 7. As the current density is increased, the water content of the membrane decreases, causing a larger ohmic drop due to its effect on conductivity. Furthermore, the profiles become more curved as the current density is increased because of the interactions between the water gradient and electro-osmotic fluxes in the membrane (see equation 11). There are two main ways in which the diffusive transport can be treated, first by dilute solution theory 
and second by concentrated solution theory. The former approach is simpler to implement and may yield sufficiently correct answers, especially in terms of current density. However, it lacks the universality and rigor of the latter approach, which can be applied for all water contents.

\subsubsection{Dilute solution theory}

Dilution solution theory starts with the use of the Nernst-Planck equation [59]

$$
\mathbf{N}_{i}=-z_{i} u_{i} F c_{i} \nabla \Phi-D_{i} \nabla c_{i}+c_{i} \mathbf{v}
$$

The first term in the expression is a migration term, representing the motion of charged species that results from a potential gradient. The migration flux is related to the potential gradient $(-\nabla \Phi)$ by a charge number, $z_{i}$, concentration, $c_{i}$, and mobility, $u_{i}$. The second term relates the diffusive flux to the concentration gradient using a diffusion coefficient, $D_{i}$. The final term is a convective term and represents the motion of the species as the bulk motion of the solvent carries it along. For one-phase treatment, the solvent is the membrane, and thus $\mathbf{v}=0$.

Dilute solution theory considers only the interactions between each dissolved species and the solvent. The motion of each charged species is described by its transport properties, namely, the mobility and the diffusion coefficient. These transport properties can be related to one another at via the Nernst-Einstein equation [59-61]

$$
D_{i}=R T u_{i}
$$

where $R$ is the ideal-gas constant and $T$ is the absolute temperature. So long as the solute species are sufficiently dilute that the interactions among them can be neglected, material balances can be written based upon the above expression for the flux (equation 8).

For the protons in the membrane, equation 8 reduces to Ohm's law, equation 6 . For the movement of water in the membrane, the Nernst-Planck equation reduces to Fick's law, 


$$
\mathbf{N}_{\mathrm{w}}=-D_{\mathrm{w}} \nabla c_{\mathrm{w}}=-D_{\lambda} \nabla \lambda
$$

because water has a zero valance. The second equality comes because the concentration and diffusion coefficient of water can be written as functions of $\lambda$ if desired, as discussed in section 4.3. The models that utilize the Fick's law treatment are interested mainly in modeling effects outside of full cells $[38,41,62-67]$. Thus, they typically assume a constant diffusivity, resulting in a linear gradient of $\lambda$ in the membrane. This allows one to estimate the water flux by just knowing the water content of the membrane at its anode and cathode interfaces.

While equation 10 stems from dilute solution theory, more rigorous membrane models incorporate the observation that the moving protons induce a flux of water in the same direction. Technically, this electro-osmotic flow is a result of the proton-water interaction, and is not a dilute solution effect since the membrane is taken to be the solvent. As shown in the next section, the electro-osmotic flux is proportional to the current density and can be added to the diffusive flux to get the overall flux of water

$$
\mathbf{N}_{\mathrm{w}}=\xi \frac{\mathbf{i}}{F}-D_{\mathrm{w}} \nabla c_{\mathrm{w}}
$$

where $\xi$ is the electro-osmotic coefficient, the number of water carried per proton in the absence of a concentration gradient. The above equation and Ohm's law have been used successfully for most of the models that treat the membrane as a single phase $[10,62,63,65,66,68-82]$. The deviations and complications in the models arise from what functions are used for the various membrane transport properties and water content (i.e., the constitutive and supporting relations but not the governing-equation framework), as discussed in section 4 . 


\subsubsection{Concentrated solution theory}

For an electrolyte with three species, it is more rigorous and almost as simple to use concentrated solution theory. Concentrated solution theory takes into account the binary interactions between all of the species. In addition, it uses a more general driving force, namely, that of chemical potential. As discussed later, this allows for the concentrated-solution-theory equations to be valid for both the diffusive and hydraulic models, the only difference being in the interpretation of the chemical potential. Consequently, if concentrated solution theory is used, the model can easily become a combination one (i.e., valid for all water contents), as discussed in section 3.2.4.

For membranes, concentrated solution theory was initially used by Bennion [83] and Pintauro and Bennion [84]. To do the analysis, one starts with the equation of multicomponent transport

$$
\mathbf{d}_{i}=c_{i} \nabla \mu_{i}=\sum_{j \neq i} K_{i, j}\left(\mathbf{v}_{j}-\mathbf{v}_{i}\right)
$$

where $\mathbf{d}_{i}$ is the driving force per unit volume acting on species $i$ and can be replaced by a chemical potential gradient of species $i$ [85], $K_{i, j}$ are the frictional interaction parameters between species $i$ and $j$, and $\mathbf{v}_{i}$ is the velocity of $i$ relative to a reference velocity (that of the membrane, which is stationary). The treatment of equation 12 can now be done in one of two ways.

The first treatment is to introduce a concentration scale and relate the frictional coefficients to binary interaction parameters

$$
K_{i, j}=\frac{c_{\mathrm{T}} x_{i} x_{j} R T}{D_{i, j}}
$$


where $D_{i, j}$ is the binary interaction parameter between species $i$ and $j, c_{\mathrm{T}}$ is the total concentration, and $x_{i}$ is the mole fraction of species $i$. Doing the above substitution into equation 12 results in the so-called binary friction model,

$$
\nabla \mu_{i}=\sum_{j \neq i} \frac{R T x_{j}}{D_{i, j}^{\mathrm{e}}}\left(\frac{\mathbf{N}_{j}}{c_{j}}-\frac{\mathbf{N}_{i}}{c_{i}}\right)-\frac{R T}{D_{i, \mathrm{~m}}^{\mathrm{e}}}\left(\frac{\mathbf{N}_{i}}{c_{i}}\right)
$$

where the $m$ denotes the interaction with the membrane and e denotes an effective property of the membrane. As discussed by Fimrite et al. $[3,86]$, this treatment is similar to that of the dusty-fluid model applied to the membrane [87-89], but accounts for the bulk movement of water in a more consistent manner using a different reference frame. This analysis is akin to the analysis of gas movement in porous media presented by Weber and Newman [90]. It should be noted that since the above treatment also implicitly accounts for convection, the model is more of a combination model and not just a diffusion one, as mentioned in the next section. A proton and water form of equation 14 can then be used to model the transport in the membrane, which results in equations similar to Nernst-Planck (equation 8), except that the cross terms are accounted for (see equations 16 and 17). The models that use the above formulation are primarily focused on modeling the conductivity of the membrane, and are discussed in section 4.3 .

While equation 14 can be used, it is somewhat complicated and requires predicting or measuring the necessary binary interaction parameters. A simpler and just as general approach is to use equation 12 , but instead of changing the frictional coefficients into binary interaction parameters, the equations are inverted instead

$$
\mathbf{N}_{i}=-\sum_{j} L_{i, j} c_{i} c_{j} \nabla \mu_{j}
$$


where the $L_{i, j}$ 's are related directly to the $K_{i, j}$ 's [90]. For the three-component membrane system, there are $1 / 2 N(N-1)=3$ independent transport properties that are needed to characterize the system. The $L_{i, j}$ 's can be related to experimentally measured transport properties using a set of three orthogonal experiments $[84,90,91]$. Doing this results in the proton and water governing transport equations,

$$
\mathbf{i}=-\frac{\kappa \xi}{F} \nabla \mu_{\mathrm{w}}-\kappa \nabla \Phi
$$

and

$$
\mathbf{N}_{\mathrm{w}}=\xi \frac{\mathbf{i}}{F}-\alpha_{\mathrm{w}} \nabla \mu_{\mathrm{w}}
$$

respectively, where $\alpha_{w}$ is the transport coefficient of water. The above equations have also been arrived at using an irreversible thermodynamics approach [92, 93].

Upon comparison of equation 16 to 6 , it is seen that the proton-water interaction is now taken into account. This interaction is usually not too significant, but it should be considered when there is a large water gradient (e.g., low-humidity or high-current-density conditions). Upon comparison of equation 17 to 11 , it is seen that the equations are basically identical in form where the concentration and diffusion coefficient of water have been substituted for the chemical potential and transport coefficient of water, respectively. Almost all of the models using the above equations make similar substitutions for these variables $[3,9,90,94-96]$.

The exceptions to this are the models of Janssen [97] and Weber and Newman [91], where the chemical potential is used directly. Janssen takes the transport coefficient as a fitting parameter, and Weber and Newman relate it to experimental data. Both models demonstrated good agreement with water-balance data under a variety of conditions. Janssen states that using a chemical-potential driving force does not necessitate making the distinction between diffusive 
or convective flow in the membrane. However, as Meyers [98] points out, by assuming the membrane system is a single phase, it cannot support a pressure difference inside it. The only way that a single-phase membrane model can have a pressure difference across it is if the chemical potential or water concentration is being altered at the boundaries. This problem is why single-phase membrane models cannot adequately describe transport for fully hydrated membranes where the driving force is the liquid pressure. For this case, one needs to use a twophase model, although the above concentrated-solution-theory equations remain the same (only the transport coefficient and chemical-potential interpretation change) as noted below.

\subsubsection{Hydraulic models}

In opposition to the single-phase treatment above are the models that assume the membrane system is two phases. This type of model corresponds to the liquid-equilibrated membrane shown in Figure 5 and Figure 6 (i.e., high water contents where there is a bulk-like liquid-water phase in the membrane). In this structure, the membrane is treated as having pores that are filled with liquid water. Thus, the two phases are water and membrane.

The addition of a second phase allows for the membrane system to sustain a pressure gradient in the water because of a possibly unknown stress relation between the membrane and fluid at every point in the membrane. However, diffusion of water becomes meaningless since the water is assumed to be pure in the models discussed here. Furthermore, unlike the models discussed above, the water content of the membrane is usually assumed to remain constant $(\lambda=$ 22) as long as the membrane remains fully liquid equilibrated and has been pretreated appropriately. For the transition case between liquid- and vapor-equilibration, see sections 3.2.4 and 4.1.1. 
The first model to describe the membrane using a hydraulic approach was that of Bernardi and Verbrugge [11, 12], which was based on earlier work by Verbrugge and Hill [99, 100]. This model utilizes a dilute solution approach that uses the Nernst-Planck equation (equation 8) to describe the movement of protons, except that now $\mathbf{v}$ is not equal to zero because they move in the separate water phase. The velocity of the water is give by Schlögl's equation [100,101]

$$
\mathbf{v}=-\left(\frac{k}{\mu}\right) \nabla p_{\mathrm{L}}-\left(\frac{k_{\Phi}}{\mu}\right) z_{f} c_{f} F \nabla \Phi
$$

where $k$ and $k_{\Phi}$ are the effective hydraulic and electrokinetic permeability, respectively, $p_{\mathrm{L}}$ is the hydraulic or liquid pressure, $\mu$ is the water viscosity, and $z_{f}$ and $c_{f}$ refer to the charge and concentration of fixed ionic sites, respectively.

In the above system, the movement of water can be attributed to a potential gradient and a pressure gradient. The movement of water by a pressure gradient is determined primarily by an effective permeability of water moving through the pore network. This approach is quite useful for describing fuel-cell systems as long as the membrane is well hydrated with a uniform water content. Such a treatment does not necessarily lend itself to describing the flux of water resulting when there is a water-activity gradient across the membrane (i.e., when the membrane is not fully hydrated). Many other models use the same approach and equations as Bernardi and Verbrugge, especially for systems wherein the membrane is expected to be well hydrated (e.g., saturated gas feeds) [102-109].

Instead of the dilute solution approach above, concentrated solution theory can also be used to model liquid-equilibrated membranes. As done by Weber and Newman [91], the equations for concentrated solution theory are the same for both the one phase and two phase cases (equations 16 and 17) except that chemical potential is replaced by hydraulic pressure and the transport 
coefficient is related to the permeability through comparison to Darcy's law. Thus, equation 17 becomes

$$
\mathbf{N}_{\mathrm{w}}=\xi \frac{\mathbf{i}}{F}-\frac{k}{\mu \bar{V}_{\mathrm{w}}} \nabla p_{\mathrm{L}}
$$

where $\bar{V}_{\mathrm{w}}$ is the molar volume of water. Furthermore, equation 18 can be arrived at from equation 12 using a similar analysis of replacing the chemical potential driving force with one of pressure [3], as mentioned above.

An interesting aspect of hydraulic models is that the water-pressure profile inside the membrane is essentially linear (instead of the curved ones seen in Figure 7). This is because the water content and consequently the properties are assumed uniform. Also, this assumption means that the net water flux in the membrane should be constant with respect to current density because all of the transport is linearly proportional to the current density. While this is true for the case of a zero pressure difference, it is not the case when there is a pressure differential because this is an extra force driving water from the cathode to the anode, as shown in Figure 8. The figure clearly demonstrates that such pressure differences can significant alter fuel-cell water management, which is one reason why differentials are sometimes used. In Figure 8, the value of $\beta$ goes to negative infinity (net water flux is from cathode to anode) as the current approaches zero by its definition (see equation 7). However, under these conditions a hydraulic model may not be valid, which is why the predicted $\beta$ values are lower than those usually observed [110]. In other words, the pressure effects are over emphasized if one only using a hydraulic-model approach. Overall, as long as water is on both sides of the membrane, the hydraulic treatment remains valid, and the effect of pressure difference is significant. 


\subsubsection{Combination models}

The two approaches above can be contrasted to one another. In the first approach, section 3.2.2, water moves by diffusion and pressure-driven flow is excluded as a separate driving force. In the second approach, section 3.2.3, only pressure-driven flow is used and there is no diffusive flow. Furthermore, the former approach assumes one phase and the latter two phases. In essence, the diffusive models describe transport in vapor-equilibrated membranes, and the hydraulic models in liquid-equilibrated ones. Thus, to model the full spectrum of water contents, some kind of superposition of the two is required $[13,14]$. In other words, a model for the transition region between the two modes is necessary.

Perhaps the easiest way to do the superposition is to combine linearly the two driving forces for water, as many models have done $[38,55,66,67,106,111-116]$

$$
\mathbf{N}_{\mathrm{w}}=\xi \frac{\mathbf{i}}{F}-D_{\mathrm{w}} \nabla c_{\mathrm{w}}-c_{\mathrm{w}} \frac{k}{\mu} \nabla p_{k}
$$

where $p_{k}$ can be the gas- or liquid-phase pressure. The first problem with the above approach is that it modifies only the water-transport equation and not the proton one. Secondly, and of more importance, the meaning of the two different gradients is somewhat muddled. In essence, this is a dilute solution approach and the two driving forces should not necessarily be valid for all water contents. Furthermore, the addition of both driving forces means that an extra variable is being added to the set of unknowns, and consequently an additional relation is required (e.g., assumption of equilibrium between the concentration and pressure). While this treatment is neither really rigorous nor consistent, it does help to fit and explain data.

Before discussing other approaches, it is of interest to examine the magnitude of the terms in equation 20. Figure 9 shows the various fluxes for a simulation where the anode is much drier than the cathode. As can be seen, the convective flux is much larger than the diffusive one, 
which is to be expected (for example, see Figure 8). The two fluxes are almost parallel, which means that the driving forces are basically linearly related. While this is more happenstance than design, it does agree with the more physical arguments discussed below. The curvature of the convective and diffusive fluxes is mainly due to the shape of the electro-osmotic flux, since the total water and proton fluxes must remain constant for the steady-state simulation (see equation 2). The electro-osmotic flux curves because the electro-osmotic coefficient is a function of water content. This flux is also much larger than the other two, and the three combine to a total flux that is close to zero, which is one reason why fuel cells operate.

Because a single-phase model cannot sustain a pressure gradient, Meyers [98] included pressure-driven flow by allowing for a discontinuity in pressure at the membrane/solution interface, even though the electrochemical potential of all soluble species is continuous. $\mathrm{He}$ argues that the additional mechanical stresses compressing the membrane should be indistinguishable from the thermodynamic pressure, and thus the thermodynamic pressure might be discontinuous at the interface. In essence, this approach is similar to using the chemicalpotential, concentrated-solution-theory approach described below, although it is not as consistent.

The most consistent and rigorous approach for modeling the transition region and the whole spectrum of water contents is to use concentrated solution theory. As noted above, such an approach accounts for both convection and diffusion in the membranes. While multiple dilute solution approaches have been used for the various water contents, the concentrated-solutiontheory approach and equations are independent of the water content. The key is using the correct parameter values and in the interpretation of the single driving force of chemical potential $[3,91$, 97]. This driving force combines those of pressure and activity 


$$
\nabla \mu_{\mathrm{w}}=R T \nabla \ln a_{\mathrm{w}}+\bar{V}_{\mathrm{w}} \nabla p_{k}
$$

Using this definition in the governing transport equations (16 and 17), one can describe transport across all water contents.

Weber and Newman [91] and later models [117-120] use the above treatment. They assume that the two transport modes (diffusive for a vapor-equilibrated membrane and hydraulic for a liquid-equilibrated one) operate in parallel with the single driving force above and single net water and proton fluxes across the membrane. Consequently, their governing transport equations remain identical for all water contents. What does change is the value of the property expressions. To model the transition region between the vapor- and liquid-equilibrated modes, they introduce a parameter known as the fraction of expanded channels,

$$
S=\frac{\lambda-\left.\lambda_{\mathrm{V}}\right|_{a_{\mathrm{w}}=1}}{\lambda_{\mathrm{L}}-\left.\lambda_{\mathrm{V}}\right|_{a_{\mathrm{w}}=1}}
$$

where $\left.\lambda_{\mathrm{V}}\right|_{a_{\mathrm{w}}=1}$ and $\lambda_{\mathrm{L}}$ are the values of $\lambda$ for the membrane in equilibrium with saturated vapor and liquid at the operating temperature, respectively. They then use this parameter to average the vapor- and liquid-equilibrated transport properties. Although averaging the two equations by this fraction is not necessarily rigorous, it has a physical basis and the correct limiting behavior (i.e., all vapor-equilibrated when there is no bulk-like water, $S=0$, and all liquid-equilibrated when there is bulk-like water throughout, $S=1$ ), with a relatively sharp transition, as expected for a phase transition. The problem with the above approach is that one has to have a method for calculating $S$. While there is no consensus, most who use this or a similar approach calculate $S$ using a capillary framework, as discussed in section 4.1.1. Another possibility is to a assume a value for $S$, such as that of the liquid saturation in contact with the membrane [121]. 
The Weber-and-Newman approach can be compared to that of assuming separate gradients (equation 20). One problem with their approach is that it violates Schröder's paradox in that a small slope in chemical potential exists across the transition region, which is not necessarily the case if the driving forces are taken to be separate. However, the violation amount is small, and, if one makes the transition region infinitesimally thick, there is a zero difference in chemical potential across the transition in harmony with Schröder's paradox. The resulting issue is that it is unknown at what chemical potential this should occur and also whether this is physically accurate. In all, while the two combination treatments are similar and capture many of the same effects, that of Weber and Newman stems from a more consistent and rigorous basis using concentrated solution theory. It also allows one to examine the full range of water contents in the membrane as shown in Figure 10.

The figure demonstrates a maximum penetration depth of liquid water into the membrane near the air inlet. This maximum is caused by the higher liquid pressure at the membrane / cathode interface, which is due to a balance between the hydrogen and air relative humidity. When there is no liquid water in the membrane, $\lambda$ decreases gradually with both positions in the membrane, showing dehydration effects similar to that in Figure 7. The transition between transport modes and the modeling of Schröder's paradox as a continuous change in water content with a very sharp transition region is also clearly shown. Finally, the figure emphasizes the need to have the anode gases humidified. Overall, only through combination models can a physically based description of transport in membranes be accomplished that accounts for all of the experimental findings. 


\subsection{Energy balance}

Consideration of temperature distributions in the membrane is important since the water content and most of the transport properties are functions of temperature. In addition, a temperature change in the membrane can cause an appreciable change in water content if the membrane is close to saturation $[88,91,122]$. Furthermore, the membrane is somewhat resistive and insulating, resulting in possibly significant temperature gradients. However, some simulations show only very minor changes in temperature through a 1-D membrane slice. This provides some justification for the often used assumption of isothermal behavior. In other words, the energy balance becomes a specification of the temperature. While most models still treat the membrane in this fashion, some have started to include nonisothermal effects and behavior $[46,47,65,71,73,79,89,102,105,111,123-125]$. Those models that are nonisothermal along the gas channel but assume that the fuel-cell sandwich remains isothermal are not discussed in this section since the membrane is essentially taken to be isothermal $[9,66$, 67, 76]. Typically, those models that include nonisothermal effects in the membrane are of higher dimensionality and contain multiple fuel-cell-sandwich layers.

Similar to the material balances, the energy balance of the membrane is relatively straightforward, and is essentially the same for the different models. In the membrane, there is heat generation and transfer. Heat is generated due to what is known as ohmic or Joule heating, and it is transferred by convection and conduction. To begin the analysis, the energy conservation equation for the membrane is written in the form $[59,126]$

$$
\rho \hat{C}_{p}\left(\frac{\partial T}{\partial t}+\mathbf{v} \cdot \nabla T\right)=-\nabla \cdot \mathbf{q}+\sum_{i} \bar{H}_{i} \nabla \cdot \mathbf{J}_{i}
$$

where various terms of the global energy balance have been ignored due to no reactions in the membrane (see section 3.1) and because it is a single system (i.e., its volume fraction is unity). 
In the above expression, the first term represents the accumulation and convection of enthalpy, respectively, where $\hat{C}_{p}$ and $\rho$ are the heat capacity and density of the membrane system, and may depend on the water content. On the right side of the equation, the first term is due to the heat flux which is related to Fourier's law

$$
\mathbf{q}=-\sum_{i} \bar{H}_{i} \mathbf{J}_{i}-k_{T} \nabla T
$$

where $\bar{H}_{i}$ is the partial molar enthalpy of species $i, \mathbf{J}_{i}$ is the flux density of species $i$ relative to the mass-average velocity, and $k_{T}$ is the effective membrane thermal conductivity. The second term on the right side of equation 23 represents enthalpy changes due to diffusion. Substitution of equation 24 into equation 23 yields

$$
\rho \hat{C}_{p}\left(\frac{\partial T}{\partial t}+\mathbf{v} \cdot \nabla T\right)=\nabla \cdot\left(k_{T} \nabla T\right)-\sum_{i} \mathbf{J}_{i} \cdot \nabla \bar{H}_{i}
$$

To get the final modeling equation, the summation on the right side of the above expression must be evaluated. While some models neglect the summation altogether $[46,65,71,73,105$, 124], others rightfully do not. While the enthalpy gradient of the water in the membrane can be taken as insignificant, that of the protons cannot and this results in the phenomenon of Joule heating $[59,127,128]$.

Joule heating is the heat that is generated due to the passage of current. Hence, it is measure of the inefficiency of the movement of current, and since the membrane is somewhat nonconductive, it is important to model it. The expression for Joule heating can be derived from the third term on the right side of equation 25 using the definition of current density and relating the gradients of enthalpy and electrochemical potential

$$
-\mathbf{J}_{\mathrm{H}^{+}} \cdot \nabla \bar{H}_{\mathrm{H}^{+}}=-\mathbf{i} \cdot \nabla \Phi=\frac{\mathbf{i} \cdot \mathbf{i}}{\kappa}
$$


The final governing energy-balance equation is determined by substituting in for Joule heating,

$$
\rho \hat{C}_{p}\left(\frac{\partial T}{\partial t}+\mathbf{v} \cdot \nabla T\right)=\nabla \cdot\left(k_{T} \nabla T\right)+\frac{\mathbf{i} \cdot \mathbf{i}}{\kappa}
$$

This equation is the one that is most often used in modeling nonisothermal behavior in fuel-cell membranes, although the convective part on the left side is sometimes ignored [47].

The inclusion of nonisothermal behavior can extend further than just adding the above energy balance. For example, one also requires that the various property values including the water content be functions of temperature. Most of the models at least partially ignore this issue and use instead effective values, which limits the analysis of nonisothermal effects. Another consideration is that, to be rigorous, nonisothermal and temperature-gradient effects need to be included in the other governing equations. For instance, because the chemical potential is undefined in a temperature field, a term must be added to account for this. Thus, a thermodynamically rigorous expression for the driving forces for transport must be used,

$$
\mathbf{d}_{i}=c_{i}\left[\nabla \mu_{i}+\bar{S}_{i} \nabla T\right]
$$

where $\bar{S}_{i}$ is the partial molar entropy of species $i$. In addition to the above driving force, expressions must be added that result in thermal diffusion [59]. However, because thermal diffusion is small relative to other effects like conduction, and there are small gradients in temperature in the membrane, this type of transport can be neglected. Similarly, the effect of diffusion on heat flux, the Dufour effect, is likewise neglected [59]. In addition, heats of mixing for ideal gases are zero, and for the other components are assumed negligible [129]. Overall, since the above effects can usually be assumed negligible, the governing conservation and equations described in sections 3.1 and 3.2 remain valid and do not require alteration. 
Figure 11 shows temperature profiles from simulations by Ramousse et al. [125]. The profiles clearly show that the membrane temperature has a maximum and its magnitude and gradient increase with current density. This occurs because there is more Joule heating and heat production as the current density increases. The magnitude of the temperature gradient is not particularly large, meaning that the membrane is relatively isothermal for this case. If one were

to operate with dry or even cool gases, then a larger and more linear temperature profile would exist [125]. Also, the more thermally conductive the cathode and anode, the flatter the membrane temperature profile. It should be noted that the simulation did not consider water evaporation/condensation, which may have significant heat effects.

\subsection{Gas crossover}

In a PEFC, oxygen and hydrogen crossover is important because of the obvious performance loss, the development of a mixed potential, and durability issues [130-132]. Furthermore, crossover becomes increasingly important as the membranes used become thinner in order to reduce their ohmic drop. Presented in this section are the governing equations and approaches which have been used to model this phenomenon. The issue of methanol crossover in DMFCs is discussed separately (see section 5.4).

Almost all models that deal with multiple fuel-cell sandwich layers at least implicitly include crossover. They do this by using a constant resistance, which is typically a fitting parameter and used to decrease the cell potential

$$
\Delta \Phi=i R_{\Omega}
$$

While in theory this resistance is just the resistance due to interlayer contact, crossover can be incorporated into it since it can be approximated to be linear with current density. This is 
especially valid for saturated feeds because the membrane is hydrated and the concentration of hydrogen is relatively fixed if there is no reformate. Furthermore, the typical crossover current density is on the order of only $5 \mathrm{~mA} \mathrm{~cm}{ }^{-2}$, and, because hydrogen is smaller than oxygen, its crossover flux is typically an order of magnitude higher; consequently, oxygen crossover is mostly ignored. While the above approach is attractive in its simplicity, it has the drawback of not predicting and accounting for the actual hydrogen and oxygen fluxes through the membrane, which cause higher reactant depletion. Since these fluxes are smaller than the fluxes due to reaction, one might consider them to be inconsequential. However, without them, one cannot predict the experimentally measured open-circuit potential, although this is not that important except perhaps in startup and shutdown scenarios. Of more importance is the contemporary concern of fuel-cell durability. To predict such effects as peroxide generation on the anode due to oxygen reduction, the crossover flux of oxygen must be known.

Before discussing explicit models of crossover, mention should be made concerning the mixed potential. A mixed potential mainly results due to hydrogen crossover and subsequent reactions at the cathode that generate and consume current locally. Some models $[42,133]$ that do not explicitly account for crossover consider the mixed potential by using an experimentally fit function for the open-circuit potential rather than a thermodynamically derived one,

$$
U^{\theta}=-\frac{\Delta G}{n F}
$$

where $\Delta G$ is the Gibbs free energy of the reaction and $n$ is the number of electrons transferred in the reaction. While this empirical fix is adequate, it is also not rigorous.

The first model to include crossover explicitly was that of Bernardi and Verbrugge [12]. They assume that there is a gas volume fraction in the membrane that remains constant. Although this does not necessarily agree with the physical picture presented and experimental 
evidence, it does allow for the calculation of gas crossover through the membrane. The way that it is included is through the convective-diffusion equation [126] (substitute equation 8 into equation 2)

$$
D_{i} \nabla^{2} c_{i}=\mathbf{v} \cdot \nabla c_{i}
$$

where the diffusion coefficients of oxygen and hydrogen in Nafion ${ }^{\circledR}$ have been experimentally determined. The use of such a simple relation can be justified since the gases in the membrane are in low concentration and do not interact significantly with each other; a dilute-solution approach is valid. The models that follow the Bernardi and Verbrugge framework treat gas crossover more-or-less the same $[102-109,111,114,134]$, although some allow for a changing gas-phase volume fraction $[135,136]$.

Other models that consider crossover have gotten around the problem of the assumption of a separate gas phase in the membrane by having the gases first dissolve in the membrane and then move by diffusion. Thus, they use both a diffusivity and a solubility. To simplify matters, Weber and Newman [91] combined these two parameters into a permeation coefficient. Thus, their proposed flux equation, which is used with the conservation-of-mass equation (equation 2) to yield the governing equation, is of the form

$$
\mathbf{N}_{i}=-\psi_{i} \nabla p_{i}
$$

where $\psi_{i}$ and $p_{i}$ are the permeation coefficient and partial pressure of species $i$, respectively. They argue that a permeation coefficient is better to use because it allows for a single variable to describe the transport, instead of two, each with their dependences on temperature, etc., which may even offset each other. Furthermore, using the above equation allows for a simple boundary condition of continuous partial pressure of the gas at the membrane interface. Even though the equation uses a partial-pressure driving force, this is due to the use of permeation coefficients. 
The real driving force is chemical potential, and the proposed physical model is not violated; there is no separate gas phase in the membrane. Finally, to be rigorous, one should add a convective term to equation 29 as was done in equation 31 . However, this convective term now signifies the amount of gas carried with the net water flux in the membrane, and a back-of-theenvelope calculation shows that such a convective flow is at least an order of magnitude less than the permeation one; it can be disregarded to a first approximation.

The permeation coefficients, like the other transport properties, are expected to depend mainly on the membrane water content and temperature. To put the permeation coefficients into context and to show agreement with the physical model of the membrane, the permeation coefficients for hydrogen for the liquid-equilibrated, dry, and saturated-vapor-equilibrated cases as a function of temperature are given in Figure 12. Also included in the graph are the values for hydrogen permeation in water and Teflon ${ }^{\circledR}$. As a point of interest, the permeation coefficient of oxygen is about $2 / 3$ that of the hydrogen coefficient. As can be seen in the figure, the permeation-coefficient values are basically bounded, with the liquid-equilibrated values higher than the vapor-equilibrated ones. The values are bounded because at higher water contents the gases mainly move through the bulk-like liquid water, and under dry conditions, the membrane is very similar to Teflon ${ }^{\circledR}$.

\subsection{Boundary conditions and solution methods}

The above governing equations require boundary conditions in order to be solved. The boundary conditions which are used vary slightly from simulation to simulation. For those simulations which consider effects in the membrane and other layers, internal boundary conditions serve as the necessary ones for the membrane equations. Thus, continuity in 
superficial flux densities and interstitial concentrations such as liquid pressure and chemical potential are used along with such variables as temperature (i.e., thermal equilibrium is assumed). For membrane-only models, the boundary conditions are typically the operating conditions like the current density and water activity at the two membrane edges. In addition, a reference-potential boundary condition is often used on one side of the membrane. Finally, there are those models that treat catalyst layers as interfaces $[74,97,118,139,140]$. For these simulations, the membrane boundary conditions can include source terms such as reactant consumption and heat generation.

Due to the complexity and interconnectivity of the governing equations and constitutive relationships, most fuel-cell models are solved numerically. To do this, a control-volume approach is used. This approach is based on dividing the modeling domain into a mesh. In between mesh points, there are finite elements or boxes. Using Taylor-series expansions, the governing equations are cast in finite-difference form. Next, the flux from the left half-box is set equal to that entering the right half-box; hence, mass is rigorously conserved. This approach requires that all vectors be defined at half-mesh points and all scalars at full-mesh points. The exact details of the numerical methods can be found elsewhere (for example, see reference [141]) and are not the purview of this chapter. The above approach is essentially the same as that used in computational-fluid-dynamics packages (e.g., Fluent ${ }^{\circledR}$ or Comsol ${ }^{\circledR}$ ) or discussed in Appendix C of Newman and Thomas-Alyea [59].

While almost all models are solved in the above fashion, analytic solutions are obtainable in certain instances $[34,68,104,116,121,135,142-144]$. The problem is that such models typically make assumptions like uniform properties, which make the solution of limited significance. Furthermore, the power of digital computers is continually progressing such that 
the computational cost of running simulations becomes manageable. However, despite the limitations, analytic solutions can provide insight, and, due to their simplicity, are very valuable for complex simulations in which the membrane is only a minor part. Another advantage is that the solutions are in closed form (i.e., the solution is general and the equations need only be solved once), and this is helpful in optimization and parametric studies.

The analytic models use essentially the dilute solution and conservation equations described above, and are isothermal. Furthermore, they normally integrate the equations directly to solve them for such parameters as the membrane resistance. This is accomplished by either assuming a net water flux through the membrane and doing the straightforward integration, or by utilizing boundary conditions such as semi-infinite diffusion. While such a boundary condition is valid only under specific fuel-cell conditions such as for minor species or at low current densities, it enables the governing equations to be solved analytically. One analytic approach that is noteworthy is to expand all of the transport properties using power series and use these expressions in solving the governing equations $[34,68,69,145]$. Such an approach allows for variable properties, although the expressions must be fit to data and are not necessarily physically accurate.

Some analytic solutions can be considered $0-\mathrm{D}$ models because they provide single equations that describe the membrane phenomena [1]. Although similar to empirical expressions (see section 2.1), this kind of $0-\mathrm{D}$ models is more rigorous as it stems from solving the governing equations, albeit with simplifying assumptions. A good example is using linear gradients in water content and Ohm's law to get expressions for the net water flux and potential drop through the membrane. Such expressions can then by incorporated into full cell models or matched at the membrane boundaries with analytic solutions of the other fuel-cell regions. 


\section{Membrane-property models}

In section 3, the governing equations and models are presented. In order to solve those equations, expressions for the various transport properties and variables are required. There are three main ways that these coefficients are determined, either empirically, semiempirically, or through modeling. Most fuel-cell models by far use empirical and semiempirical expressions. Such expressions are experimentally obtained, and the difference between the two is that semiempirical expressions use some theory and perhaps other values to guide the form of the expressions. The models for the various transport parameters and ab-initio simulations for uptake, etc., are largely microscopic in nature; they have recently been reviewed in the literature [4] and are not discussed in this chapter. There are three main sets of parameters which are required and discussed in this chapter. The first, and perhaps most important, is the determination of the value of the water content of the membrane, $\lambda$. Second, is the way in which swelling and dimensional changes are considered in modeling, which is related to the water content. Finally, transport-property models and expressions are discussed.

\subsection{Water content}

As seen in the modeling equations, the water content is a key variable. Not only can it be a driving force and integral part of fuel-cell water management, but also it is the crucial parameter that almost all property expressions are related to, such as conductivity, as discussed in section 4.3. Because of the water content's importance, it has received more modeling attention than other membrane properties. Such models span from simple curve fits to more elaborate chemically based ones. All of the models seek to predict and explain at least part of the uptake 
isotherm as shown in Figure 4, including perhaps the higher uptake from liquid water than from saturated vapor (i.e., Schröder's paradox). The various modeling approaches for uptake along with Schröder's paradox are examined below. Before proceeding with the discussion, one should note that all of the models below are trying to predict the water content, which is a property that goes into the governing equations. In essence, these models are speciation ones that compliment the macrohomogeneous approaches and equations discussed in section 3 .

The majority of fuel-cell models use an empirical curve fit for the uptake isotherm. More than anything else, the polynomial fit of Springer et al. [10] is used

$$
\lambda=\left\{\begin{array}{lr}
0.043+17.81 a_{\mathrm{w}}-39.85 a_{\mathrm{w}}^{2}+36 a_{\mathrm{w}}^{3} & \text { for } 0<a_{\mathrm{w}} \leq 1 \\
14+1.4\left(a_{\mathrm{w}}-1\right) & \text { for } 1<a_{\mathrm{w}} \leq 3
\end{array}\right\}
$$

The fit is for an isotherm at $30^{\circ} \mathrm{C}$. The first part represents the uptake isotherm, and the second term is to handle the experimentally observed higher water contents with liquid water. This latter expression is a linear interpolation from $\lambda=14$ to $\lambda=16.8$ at an activity of water of 3 . Obviously, such a fit does not make sense due to the use of supersaturated activities. In essence, the curve fit violates Schröder's paradox by having the activity and thus chemical potential of water change. While the polynomial fit is not rigorous, it does allow ease of use in modeling, especially when one might have to take the derivative of the curve (see section 4.3). A problem with the fit is that it does not take into account the observed lower uptake at higher temperatures (see for example references [146-148]), although this can be done with other curve fits or by extrapolating the curve if some data points are known at higher temperatures [149]. Finally, there are some models that use the same approach except use a fourth-order polynomial [86, 133]. 
Equation 33 has a maximum water content of $\lambda=16.8$ for essentially a liquid-equilibrated membrane. The reason why this is used instead of the often reported value of 22 is that it was what was experimentally observed by Springer et al. However, the uptake is known to depend on the pretreatment procedure due to the ability of the polymer to reorient itself as its glasstransition temperature is reached around $100^{\circ} \mathrm{C}$. Thus, the value of 16.8 is valid for an " $\mathrm{N}$ " or normal-form membrane, and 22 is good for an "E" or expanded-form membrane [17, 19]. Most pretreatment procedures today ensure that the membrane is as close to its $\mathrm{E}$ form as possible due to its higher conductivity. However, it is unknown how fuel-cell assembly and compression affect the water uptake and membrane form.

Almost all models assume local equilibrium between the water vapor outside and water in the membrane; this is explicitly accounted for in the models discussed below. However, due to the long hydration times of the membrane and the possible presence of a diffusion layer, masstransfer resistances can be incorporated if desired [71, 82, 149, 150]. For the hydraulic models, the water content is not as important since the membranes are assumed to be fully liquidequilibrated. An exception to this is the model of Eikerling et al. $[135,136]$ as mentioned in section 3.2.4. This model allows for the existence of swollen and nonswollen pores, much like the expanded and collapsed channels of Weber and Newman [91]. Such an approach allows for the full range of water contents to be modeled, as discussed later.

Before proceeding to introduce the more detailed chemically and thermodynamically based uptake models, some mention should be made of the more microscopic cluster models. Typically, these models examine the interactions and forces within a cluster and try to predict cluster size and hence swelling and water uptake. While most of these models are complex and involve ab-initio approaches [4], there are some that discuss the phenomena from a macroscopic 
approach [151-153] that is in line with the focus of this review. These models examine the electrostatic and elastic energies and do a force balance to yield the amount of water in a cluster. This is somewhat akin to an osmotic-pressure analysis. Like most of the more microscopic models, these models provide insight into the membrane morphology and how swelling and water uptake are related and can be modeled, but they are not really useful for full-cell modeling.

The most useful set of uptake models are those that strike a balance between robustness and complexity $[31,76,88,91,94,95,122,154,155]$. They can explain and predict water uptake as a function of operating conditions and membrane properties, but are macroscopic and wieldy enough to be used in various simulations. They are typically chemically and physically based. The simplest of these models may be that of Thampan et al. [88], who use a Brunauer-EmmettTeller (BET) equation to fit the curve. Such an approach allows one to fit physically meaningful parameters, such as those dealing with multiple water-layer coverages and energies. This approach also allows for consideration of temperature effects on uptake. As a general point, almost all of the chemically based models include temperature effects, which are known to occur but are seldom included in membrane modeling, as mentioned above.

The rest of the chemical models utilize thermodynamics and chemical equilibria explicitly. In the first two, chemical equilibrium is assumed between protons and water with a hydronium ion that is bound or solvated to the sulfonic acid site. This equilibrium is based on the assumption that some water is tightly bound to the protons and sulfonic acid sites in the membrane $[4,13,22,156]$. In the last one, different ionization and hydration steps are considered, although only the primary one is used.

In the chemical model of Meyers and Newman [18], the equilibrium is used to relate the electrochemical potentials of the species inside the membrane 


$$
\mu_{\mathrm{H}^{+}}+\mu_{\mathrm{w}}=\mu_{\mathrm{H}_{3} \mathrm{O}^{+}}
$$

which are also subject to the boundary condition

$$
\mu_{\mathrm{w}}-\mu_{\mathrm{w}}^{\mathrm{ref}}=R T \ln a_{\mathrm{w}}
$$

where $\mu_{\mathrm{w}}^{\text {ref }}$ is the reference chemical potential, or the chemical potential of water at unit activity, and the activity is that of the water vapor outside the membrane. Expressing the electrochemical potentials according to the thermodynamic basis of Meyers and Newman [18] for multicomponent transport in a polymer-electrolyte membrane, along with electroneutrality and a mass balance on water in the membrane, leads to two equations that must be solved simultaneously

$$
\begin{aligned}
& a_{\mathrm{w}}=K_{2}\left(\lambda-\lambda_{\mathrm{H}_{3} \mathrm{O}^{+}}\right) \exp \left[\phi_{2} \lambda_{\mathrm{H}_{3} \mathrm{O}^{+}}\right\rfloor \exp \left[\phi_{3} \lambda\right] \\
& K_{1}=\left(\frac{\lambda}{\left(1-\lambda_{\mathrm{H}_{3} \mathrm{O}^{+}}\right)} \lambda_{\left(\lambda-\lambda_{\mathrm{H}_{3} \mathrm{O}^{+}}\right.} \exp \left[\phi_{1} \lambda_{\mathrm{H}_{3} \mathrm{O}^{+}}\right] \exp \left[\phi_{2} \lambda\right]\right.
\end{aligned}
$$

where $\lambda_{\mathrm{H}_{3} \mathrm{O}^{+}}$is the moles of hydronium ions (or bound water) per mole of sulfonic acid sites, the $K$ 's are essentially equilibrium constants, and the other parameters are groupings of standard states and binary interaction parameters, and are used as physically meaningful fitting parameters [18].

To account for temperature and the much stronger solvation and interaction energies at low water contents, Weber and Newman [91] modified the above model. They fit the temperature dependence of the equilibrium constant, $K_{2}$, to experimental data. For the low water contents, they use a simple empirical expression to correct the uptake. Although such a correction is not rigorous, the lack of data at low water contents and the unimportance of this region during fuelcell operation and simulation justify the approach. 
Using the same equilibrium concept as above, Futerko and Hsing [122] and Tsonos [155] use a Flory-Huggins approach [157] instead of one based explicitly on a single Gibbs function [18, 158]. Such an analysis results again in two equations that must be solved simultaneously

$$
\begin{aligned}
& a_{\mathrm{w}}=\left[1-\phi_{\mathrm{m}}^{\prime}\right] \exp \left[\left(1-\frac{\bar{V}_{\mathrm{w}}}{\bar{V}_{\mathrm{m}}}\right) \phi_{\mathrm{m}}^{\prime}+\chi \phi_{\mathrm{m}}^{\prime 2}\right] \\
& K=\frac{\lambda_{\mathrm{H}_{3} \mathrm{O}^{+}}}{\left(1-\lambda_{\mathrm{H}_{3} \mathrm{O}^{+}}\right) a_{\mathrm{w}}}
\end{aligned}
$$

where $K$ is the equilibrium constant, $\chi$ is the Flory interaction parameter and is a linear function of $\phi_{\mathrm{m}}^{\prime}$, and $\phi_{\mathrm{m}}^{\prime}$ is a modified volume fraction of the membrane that accounts for association of the hydronium ions

$$
\phi_{\mathrm{m}}^{\prime}=\frac{\bar{V}_{\mathrm{m}}+\lambda_{\mathrm{H}_{3} \mathrm{O}^{+}} \bar{V}_{\mathrm{w}}}{\bar{V}_{\mathrm{m}}+\lambda \bar{V}_{\mathrm{w}}}
$$

where $\bar{V}_{\mathrm{m}}$ is the partial molar volume of the dry membrane

$$
\bar{V}_{\mathrm{m}}=\frac{E_{W}}{\rho_{\mathrm{m}, \mathrm{o}}}
$$

where $\rho_{\mathrm{m}, \mathrm{o}}$ is the density of the dry membrane and $E_{W}$ is the membrane's equivalent weight. The above definition of $\phi_{\mathrm{m}}^{\prime}$ accounts for membrane swelling effects as discussed in section 4.2. Temperature is considered by fitting the values of $K$ and $\chi$ to data.

The final approach to be mentioned is that of Datta and coworkers [31, 154]. It is perhaps one of the most complete because it allows one to calculate the entire water-uptake curve including the liquid-equilibrated value. Their model is developed using a similar Flory-Huggins approach and chemical equilibrium between water vapor, bound (i.e., hydronium), and free water molecules. The main difference from those above is that Datta and coworkers also incorporate 
the energetic effects due to swelling and any interfaces in a membrane pore on the chemicalpotential expressions.

For the membrane pressure expression, their first model uses a spring constant as a fitting parameter, and their later model refines this and uses a swelling model $[154,159]$

$$
\Pi_{\mathrm{m}}=\frac{2}{3} G\left(\phi_{\mathrm{m}}^{1 / 3}-\phi_{\mathrm{m}}^{7 / 3}\right)
$$

where $\Pi_{\mathrm{m}}$ is the pressure value, $\phi_{\mathrm{m}}$ is the volume fraction of the membrane and is given by equation 38 with the second (hydronium) term on the numerator neglected, and $G$ is the shear modulus of the membrane and is a function of water content

$$
G=\frac{1}{3} E_{0} \exp \left(-2.1753\left(\frac{\lambda \bar{V}_{\mathrm{m}}}{\bar{V}_{\mathrm{m}}+\lambda \bar{V}_{\mathrm{w}}}\right)\right)
$$

where $E_{0}$ is the dry-membrane value of the Young's modulus. For the interfacial pressure relationship, they use the Young-Laplace equation [160]

$$
\Pi_{\gamma}=-\frac{2 \gamma \cos \theta}{r}
$$

where $\gamma$ is the surface tension of water, $r$ is the membrane pore radius, and $\theta$ is the contact angle of water with the membrane surface. These pressures are then incorporated into the FloryHuggins approach, and the two equations to be solved simultaneously become

$$
\begin{aligned}
\ln \left(\frac{\bar{V}_{\mathrm{w}}\left(\lambda-\lambda_{\mathrm{H}_{3} \mathrm{O}^{+}}\right)}{\bar{V}_{\mathrm{m}}+\bar{V}_{\mathrm{w}} \lambda}\right)+\left(1-\frac{\bar{V}_{\mathrm{w}}}{\bar{V}_{\mathrm{m}}}\right) \phi_{\mathrm{m}}^{\prime}+\chi \phi_{\mathrm{m}}^{\prime 2}-\ln a_{\mathrm{w}} & =-\frac{\bar{V}_{\mathrm{w}}}{R T}\left[\frac{2}{3} G\left(\phi_{\mathrm{m}}^{1 / 3}-\phi_{\mathrm{m}}^{7 / 3}\right)-\Pi_{\gamma}\left(1+\frac{\bar{V}_{\mathrm{m}}}{\bar{V}_{\mathrm{w}} \lambda}\right)\right] \\
\lambda_{\mathrm{H}_{3} \mathrm{O}^{+}} & =1800 \frac{a_{\mathrm{w}}}{1-a_{\mathrm{w}}}\left(\frac{1-6 a_{\mathrm{w}}^{5}+5 a_{\mathrm{w}}^{6}}{1+999 a_{\mathrm{w}}-1000 a_{\mathrm{w}}^{6}}\right)
\end{aligned}
$$

where the second equation deals with the way they have assumed chemical equilibrium. The same set of equations works for both liquid- and vapor-equilibrated membranes, with the 
difference being that the last term in the top equation, $\Pi_{\gamma}$, does not exist for the liquidequilibrated case. The physical reason is that there is no meniscus or phase boundary with liquid water as there is for vapor. This concept and the above approach is discussed in more depth below. Although most of the various parameters in the above set of equations have known temperature functions, they do not explore temperature effects.

It is readily apparent that all of the more detailed approaches of modeling water uptake involve the same phenomena, namely, chemical equilibria of bound, free, and vapor water, as well as a thermodynamic basis. The approaches are mainly just different in how the thermodynamic functions are developed and used. The approach taken, especially the two-phase one of Datta and coworkers, are all on the right track, although none is truly definitive.

As a further point of comparison, one can discuss and compare the various isotherm models including the empirical and semiempirical expressions. Since all of them are fit to experimental data at $30^{\circ} \mathrm{C}$, they all do a good job there. However, when one changes the conditions (e.g., membrane equivalent weight, temperature, etc.) to those not where the data was fit, the more rigorous chemical-modeling equations are more accurate and predictive. The sensitivity of the water uptake is also explored in the relevant papers in terms of the physical parameters such as the Flory interaction parameter $[122,154]$, the dissociation constant $[18,31,154]$, and the membrane properties [154]. The main drawback to using the more rigorous models is that they require the solution of two simultaneous equations (i.e., there is no single closed-form equation). This does slow down computation time and necessitates numerical solution and derivatives, but the physical and realistic gains offset these issues. 


\subsubsection{Vapor- to liquid-equilibrated transition (Schröder's paradox)}

As alluded to throughout this chapter, there are physical and property changes depending on the reservoir phase in contact with the membrane, i.e., Schröder's paradox. It is known as a paradox because one has seemingly two different membrane states even though the chemical potential of the water has not changed. However, contemporary thinking is that it is essentially a phase transition that is occurring in the membrane. Thus, the liquid- and vapor-equilibrated membranes remain in equilibrium even though they have slightly different internal microstructures. While vapor is essentially excluded from the membrane, the two phases are more akin to bulk-like and associated or bound water in the nanopores of the membrane. One can also think of this as collapsed and expanded channels or pores as introduced in sections 2.3 and 3.2.4. The collapsed channels can be thought of regions with associated water, and the expanded ones as where there is a bulk-like liquid-water phase. Such a physical picture is helpful in understanding how to account for the paradox.

Schröder's paradox is an observed phenomenon that should be considered in any membrane model where the membrane does not remain fully hydrated or dehydrated. There are various methods to account for the paradox. The easiest way is to ignore it, which a majority of the models do. Next, it can be treated as a discontinuity, which results in both numerical problems and deciding the discontinuity location. Another approach is to assume a functional form of the water content such that $\lambda$ and $a_{\mathrm{w}}$ continue to increase (see equation 33). However, this violates Schröder's paradox to a significant degree. Finally, there are the more phenomenological and rigorous ways to account for it. These methods are essentially all based on using capillary phenomena and equations and methodology from two-phase flow and vapor-liquid equilibrium. 
There are three main groups who have used such an approach, Weber and Newman [91], Eikerling et al. [136], and Datta and coworkers [31, 154].

The key way to model the change in water content is to use a parameter that is essentially the fraction of expanded channels (equation 22) as discussed in section 3.2.4. Basically, this factor linearly interpolates between the water content of the vapor-equilibrated membrane with that of the liquid-equilibrated one. While it is a linear function in terms of water content, it is not necessarily in terms of activity, pressure, or other variables. This approach allows for a continuous transition between vapor- and liquid-equilibrated membrane water contents, although the transition can be relatively sharp, which is not unexpected for a phase-change-type behavior. The key difference between the models is essentially how they determine $S$.

The most microscopic of the three approaches is that of Eikerling et al. They use a random network of collapsed or expanded pores with a pore-size distribution. They assume that impregnation by liquid water is easier than condensation, although the pores may contain vapor. To determine how the water moves and if the pores are expanded, they use capillary equations of the form of equation 42 , where $\Pi_{\gamma}$ is the capillary pressure. Their model is aimed more at percolation and water permeability and proton conductivity than water uptake, although it can easily be modified to yield uptake.

Weber and Newman utilize an approach where $\Pi_{\gamma}$ is calculated using a form of equation 42 but where it is applied throughout the channels inside the membrane and where the parameters have been given a different meaning. In their treatment, the contact angle and surface tension represent the various interactions between bulk-like water and the membrane-channel surface such as membrane deformation and coulombic repulsion and attraction energies between 
membrane, bound water, and free or bulk-like water. For this case, equation 42 is simplified to contain only a single unknown constant, $\Gamma$, that accounts for these various energetic interactions

$$
r_{\mathrm{c}}=\frac{\Gamma}{p_{\mathrm{L}}}
$$

where $\Pi_{\gamma}$ has been replaced by the liquid pressure and $r_{\mathrm{c}}$ is the incipient expanded channel radius such that smaller radii are not expanded (no bulk-like water phase) and larger ones are. $\Gamma$ is assumed to be a function of temperature in the same way as the elastic modulus and Flory parameter are assumed to be (i.e., inverse relation) [122, 161]. To calculate $S$, a bundle-ofcapillaries approach is used that integrates a channel or ionic-domain size distribution from infinity to $r_{\mathrm{c}}$ [119], resulting in

$$
S=\frac{1}{2}\left[1-\operatorname{erf}\left(\frac{\ln r_{\mathrm{c}}-\ln (1.25)}{0.3 \sqrt{2}}\right)\right]
$$

This approach has a problem that Schröder's paradox is violated when a single driving force is used, as mentioned in section 3.2.4. The reason is that $S$ changes over a small range of chemical potential due to the assumption of a channel-size distribution. To correct this requires either knowing which chemical potential the transition occurs at (e.g., that of bulk liquid to vapor water) or using something akin to a Kelvin equation which would thermodynamically support a chemical-potential gradient and coexistence of both expanded and swollen channels $[160,162]$. More experimental data is required to determine the validity of the approach and refine it.

The final model that considers the transition region is that of Datta and coworkers discussed in the previous section. This treatment is similar to those above except that the capillary phenomena occur at the boundary of the membrane channel and not within its volume like in the other two methods. Their approach also accounts for the swelling pressure explicitly. In a 
certain sense, they determine $\Gamma$ more explicitly. In their approach, the last term on the right side of the top expression in equation 43 is absent when in contact with liquid (this is similar to making $S=0$ for vapor and $S=1$ for liquid). Consequently, the liquid-equilibrated uptake is higher than the vapor-equilibrated one. In other words, the meniscus that forms when in contact with water vapor and inerts results in an additional energy compared to the liquid-equilibrated case that the membrane must equilibrate with. While the approach and model development of Datta and coworkers is the most consistent, rigorous and provides good insight into the paradox, it has not been used in a full-cell simulation. In addition, it is unknown what occurs when liquid is on one side and vapor on the other (i.e., where is the phase-transition point?).

The approach of Datta and coworkers and those of the other treatments could be brought together. This would involve making the $\Pi_{\gamma}$ term in equation 43 a function of some variable like liquid pressure and not just the reservoir phase. In addition, a pore-size distribution should probably be added to allow for a slightly more gradual transition. Experimentation needs to be undertaken to prove such a model. For example, this model would predict a disappearance of the paradox as the system pressure reaches the vapor pressure due to the disappearance of the inert gases and the meniscus, or even different water uptake from helium than from air. Overall, there is still a need to understand the vapor- to liquid-equilibrated phase transition and the nature of Schröder's paradox. There is opportunity to relate it in more depth to polymer phase segregation, ionic and nonionic moieties, membrane elastic properties, and operating conditions.

\subsection{Membrane swelling (thickness)}

It is well documented that Nafion ${ }^{\circledR}$ and other PEMs swell to a significant extent, which is related to their water uptake as discussed above. In fact, liquid-equilibrated Nafion ${ }^{\circledR}$ swells 
around 60 to $70 \%$ in volume compared to its dry dimensions. The dimensional changes are proportional to the water content, and, upon examination of the uptake isotherm (Figure 4), seem to correspond with the existence of loosely bound and bulk-like water, in agreement with the physical model (see section 2.3). These dimensional changes are important because they directly impact the magnitude of the gradients and the concentration values in the membrane. The thickness is key because many models treat the sandwich and membrane in 1-D, and thus the potential drop and net water flux can change dramatically if the thickness does. The latter will change because the water gradient-flux is affected but the electro-osmotic flux is not since it is proportional to the current density. In terms of modeling, the detailed models for water uptake described in section 4.1 already basically consider the volume change effect of swelling.

As discussed, both the volume change and the thickness change are important for membrane modeling. These effects have been treated by various means in the literature by three main approaches. The simplest treatment is to assume a volume and thickness of the swollen membrane, normally based on experimental data. This assumption is fine as long as there is not a large change in water content across the membrane and the value was chosen correctly. The first models to use this approach were by Verbrugge and coworkers [11, 12, 163], who examined a fully liquid-equilibrated membrane. They used factors of 1.29 and 1.72 for the membrane thickness and volume, respectively. Many others have used this approach, typically with the same values for the thickness and volume $[62,64,65,82,97,103,104,108,112,164,165]$.

The next approach accounts implicitly for swelling by folding it into the governing equations and properties using a coordinate transformation. The concept is to transform the actual thickness coordinate, $z$, into one that is pegged to the dry thickness of the membrane, $z^{\prime}$. Doing this allows for swelling to be considered but not actually tracked. Hence, all of the properties 
like diffusion coefficients as well as concentrations are cast in this new form, and the dry membrane conditions used. This approach is by far the most often used [38-40, 66, 67, 70, 77$79,81,111,120,164,166,167]$, especially in those models that assume a linear gradient in the membrane and are just solving the dilute solution equations, as it strikes a balance between complexity and ease of use.

The transformation can be done in various ways. Springer et al. [10] popularized using an expansion factor of

$$
z^{\prime}=\frac{z}{1+s \lambda}
$$

where $s$ is the swelling factor and has a value of $s=0.0126$. Using their transformation, water concentrations are written as

$$
c_{\mathrm{w}}=\frac{1}{\bar{V}_{\mathrm{m}}} \frac{\lambda}{1+s \lambda}
$$

and the diffusion coefficients also have to be recalculated [10]. Other transformations include using either the change in density $[90,96]$ or the total volume with water content, where one can assume constant and additive partial molar volumes [29, 168-171],

$$
V=\bar{V}_{\mathrm{m}}+\lambda \bar{V}_{\mathrm{w}}
$$

Incidentally, such an assumption is used in many of the more rigorous uptake models discussed in section 4.1 (see equation 38 for example).

While the above approach does a good job in accounting for swelling, it is somewhat complicated and requires careful implementation to ensure that all terms and parameters are transformed. It also allows for different volumes and thicknesses when there is a gradient of water content in the membrane. Such a "local" swelling effect is somewhat unphysical since the membrane length is a uniform, albeit unknown a priori, value. However, applied correctly, the 
approach is numerically convenient because the thickness of the membrane remains uniform in the modified coordinate system.

A more straightforward and rigorous, albeit more numerically intensive, approach is to account for swelling explicitly [88, 91, 117, 135, 149, 161, 172]. Essentially, this is done by doing a mass balance on the membrane and using the above wet-volume expression (equation 48) with the average membrane water content. That expression is used to alter all of the variables that are a function of the water volume fraction or concentration. To account for the thickness change of the membrane, the average water content is again used

$$
l=l_{\mathrm{o}}\left(1+0.36 \frac{\hat{\lambda} \overline{V_{\mathrm{w}}}}{\bar{V}_{\mathrm{m}}}\right)
$$

where $l$ and $l_{\mathrm{o}}$ are the swollen and dry membrane thicknesses, respectively, and $\hat{\lambda}$ is the average value of $\lambda$ in the membrane. In the above expression, a value of 0.36 is used instead of 0.29 due to the slight anisotropy in membrane swelling $[29,91,169,173]$. Since $\hat{\lambda}$ is not known a priori, one must iterate over the entire simulation until the value converges [91, 118].

To get around this obstacle, Newman and coworkers $[172,174]$ developed the following set of two governing differential equations. The first equation is an expression of the average water content as an integral

$$
\hat{\lambda}=\frac{1}{l} \int_{0}^{z=l} \lambda(z) \mathrm{d} z=\int_{0}^{1} \lambda(\varsigma) \mathrm{d} \varsigma
$$

where the equation has been nondimensionalized. While this provides the first equation, the second arises from the thickness being a scalar quantity that is uniform

$$
\frac{\mathrm{d} l}{\mathrm{~d} \varsigma}=0
$$


These two equations are solved with the swelling boundary condition given by equation 49 and applied at $\varsigma=1$. Treatment of swelling in the above fashion ensure conservation of the membrane mass, as discussed by Meyers and Newman [174].

One question that may arise is does the membrane swell inside of the fuel cell where it is under compression. To answer this question, Weber and Newman [161] used a simple stress analysis along with their membrane model to predict membrane expansion. They determined that the membrane essentially swells freely within a PEFC, i.e., the membrane pushes against the other sandwich layers and expands. This is because of its prodigious water uptake and the large force required to compress it. If desired, a fractional or degree-of-constraint term can be added to the equations above in determining the membrane thickness and volume to account for partial swelling. Such a parameter would then be determined through a stress balance.

\subsection{Transport properties}

The transport properties used are different depending on which modeling approach is taken. Normally they involve a proton diffusion coefficient or conductivity, an electro-osmotic coefficient, a permeability, a water diffusion coefficient, and/or a transport coefficient. The macroscopic property models typically apply the underlying governing-equation framework (as introduced in section 3) and apply more constitutive relations like speciation in order to examine the specifics of a property. Often, the analysis is done using concentrated solution theory since it is the most appropriate choice, especially for looking on a detailed level. While the outcome of most of these models is to yield parameter values or simple expressions for use in the governing macrohomogeneous equations, they can be useful in membrane design and optimization analysis. These models are also typically much more complex than the water-uptake ones describe above. 
The discussion below is loosely separated based on the parameter being discussed, although many are interrelated. Due to the large numbers of expressions used for the various parameters by the various models, only general comments are made about them and the discourse is mainly about the macroscopic-modeling issues of them. While in principle these issues could extend to the experimental techniques that are used to measure the properties since many require models and detailed theoretical analysis, this is beyond the scope of this review and not discussed. Also not discussed are models and property expressions for minor components such as crossover gases, since these either have not been modeled or are mentioned elsewhere in this chapter.

Before moving on, mention should be made about the relationship between these property expressions and Schröder's paradox. Since it is known that the different water contents and membrane structures yield different properties, this has to be considered. Whether one is averaging the property values for a single equation or using multiple driving forces or a single modeling treatment, the experimentally observed effects should be included in the macroscopic model. Most frequently, these effects are incorporated by having different property expressions for different water contents. An example is the electro-osmotic coefficient, which displays a large increase above vapor-equilibrated water contents, or the water diffusion coefficient, which is often separated in up to four different expressions depending on the water content of the vapor-equilibrated membrane. Once again, due to the number of expressions, the above issue is only briefly touched upon below, and for a more detailed discussion of Schröder's paradox, see section 4.1.1.

The ionic conductivity of the membrane is essentially a measure of the proton movement in the membrane. The conductivity can be defined in a certain fashion using the Nernst-Einstein equation (equation 9) for the mobility 


$$
\kappa=\frac{F^{2} D_{\mathrm{H}^{+}} c_{\mathrm{H}^{+}}}{R T}
$$

This equation implies that protons are the only mobile charge carries in the membrane, which is typically the case, although the presence of anions will affect the conductivity (see section 5.2 and equation 76). In the membrane, protons move both by the vehicle (i.e., molecular diffusion) and Grotthuss (i.e., through proton complexes and reorientations) mechanisms as mentioned in section 2.3 and reviewed in reference [4].

The value of the conductivity has been measured by many techniques and in many laboratories (for examples, see reference [175]). The resulting empirical expressions are often used in modeling. In addition, semiempirical expressions have been used. Such models explain the temperature dependence of the conductivity by the change in the equilibrium constant for the dissociation of the sulfonic acid sites and the activation energies for the Grotthuss and vehicle mechanisms [88, 91, 176]. They also ascribe the experimentally observed almost linear dependence on water content to the formation of less bound water and percolation effects. The latter was first used for Nafion ${ }^{\circledR}$ by Hsu and coworkers [25], where the volume fraction of water in the membrane is used to determine the conductivity. While this gives some meaning to the conductivity and agrees with the experimental data by showing a several order-of-magnitude change in the conductivity at very low water contents, it has shortcomings such as the fact that it cannot predict the flattening and decrease of the conductivity at higher water contents approaching infinite dilution (i.e., the polymer is dissolved in an infinite amount of water). Furthermore, the conductivity change is not necessarily due to network percolation but perhaps just to the existence of more mobile protons (i.e, those outside the inner hydration shells of the sulfonic acid sites, see Figure 5). A more rigorous (compared to just using some general expressions fit to data) percolation-type model for conductivity is presented by Eikerling et al. 
$[135,136,156]$, who use statistical relations, effective medium approximations, and different pore structures. They also summarize various proton-conduction mechanisms and examine single-pore or microscopic models of conduction.

While most of the conductivity models fall in the realm of microscopic models, which examine detailed potential and ion distributions as mentioned later and in section 5.2, there are some that are more macrohomogeneous. The main approach of these models is using concentrated solution theory, and in terms of a binary-friction model (equation 14) $[3,86,88$, 89], as discussed in section 3.2.2.2. For visualization purposes of the various species, one can use Figure 6. To apply this model to conductivity, a water-uptake model as describe in section 4.1 and a dissociation model are required.

For the dissociation model, protons are considered both from the sulfonic acid site and from water complexes such as hydronium ions. An expression for the degree of dissociation, $\alpha$, can easily be found through chemical equilibria [88], resulting in

$$
\alpha=\frac{(\lambda+1)-\sqrt{(\lambda+1)^{2}-4 \lambda\left(1-1 / K_{A, C}\right)}}{2\left(1-1 / K_{A, C}\right)}
$$

where $K_{A, C}$ is the equilibrium dissociation constant and is a function of temperature. This equation can be combined with the transport equations to yield an expression for the conductivity in the form

$$
\kappa=\frac{F^{2}}{R T \lambda \bar{V}_{\mathrm{w}}}\left(f-f_{0}\right)^{1.5} \mathrm{f}\left(\alpha, D_{i, j}, \lambda\right)
$$

where the second part is a Bruggeman and percolation expression, as discussed above, where $f$ is the volume fraction of water in the membrane, and the last part is some function of the dissociation constant and the three binary interaction parameters between water, membrane, and 
protons. Equation 54 along with an isotherm model is then fit to experimental data to determine the necessary parameters. The resultant equation and parameter values provide insight into conduction such as the limiting step and energy required, effect of equivalent weight, etc.

Almost all of the macroscopic membrane models utilize the electro-osmotic coefficient as one of the key experimentally measured parameters. This coefficient, sometimes termed the electro-osmotic drag coefficient, is the transport number of water in the membrane. It is a measure of the number of water molecules that are carried with each proton in the absence of a concentration gradient. The electro-osmotic coefficient arises mainly due to the complexes that solvate the protons and the vehicle mechanism, and depends solely on temperature and water content, at least macroscopically. Most models use empirical (e.g., depends linearly on water content at high water content) or semiempirical expression for the coefficient, although the latter is much preferred.

An example of a semiempirical argument is as follows [91]. For a vapor-equilibrated membrane, the electro-osmotic coefficient has a value close to unity since basically only a hydronium ion is moving through the membrane. However, for a liquid-equilibrated membrane, the coefficient has a much larger value due to the ability to form extended complexes like Zundel, $\mathrm{H}_{5} \mathrm{O}_{2}{ }^{+}$, and Eigen, $\mathrm{H}_{9} \mathrm{O}_{4}{ }^{+}$, ions with the bulk-like-water molecules. Furthermore, it has a dependence on temperature with an activation energy that describes the data and stems from the amount of energy needed to break a hydrogen bond in the bulk-like water in Nafion ${ }^{\circledR}$ [91], which is the limiting step in the Grotthuss mechanism $[28,156,177]$.

There are also some models that try to predict the electro-osmotic coefficient or use the electro-osmotic flux to determine membrane properties and structural aspects [4, 178-181]. Since electro-osmotic flow is intricately related to proton conduction, the conductivity models 
mentioned above can in principle determine the electro-osmotic coefficient with some minor adjustments. Of the models that only examine electro-osmotic flow, all of them except that of Breslau and Miller [178] use a traditional electro-osmotic approach of examining the fluid flow due to that in the double layer next to the membrane surface inside of a pore (for example, see reference [59]). Such an approach calculates the potential distribution and proton concentration in a membrane pore with a charged wall. This is accomplished by solving Poisson's equation (equation 73) with a distribution function. Once solved, hydrodynamic equations are used to determine the velocity of the water due to the presence of the charged particles and an electric field. This approach is discussed in some more detail in section 5.2, and the reader is referred to a review on the subject [4] and chapter 9 of reference [59]. As noted, Breslau and Miller use a different approach. They treat the water in a more macroscopic way in terms of hydrodynamic forces and drag. Their model and approach is relatively simple, and is notable in that it was one of the first to treat this subject in fuel-cell membranes.

The other form of water movement in the membrane is due to the gradient flux. For convective or hydraulic flow, an experimentally measured value for the permeability is used, which can be made into a transport coefficient by substitution of pressure with chemical potential [91]. The reason for just using such an empirical approach is that the membrane is typically assumed to remain fully hydrated for this case. An exception is the model of Eikerling et al. [135], which essentially treats the membrane as being a porous medium in which gas and liquid can flow. They then use statistical and percolation arguments along with capillary and twophase flow equations to determine an effective permeability that varies with water content. In this fashion, they treat the whole water-content range with a single transport coefficient, although they do have a separate gas-phase that exists within the membrane. 
Similar to the permeability, those models that utilize a water diffusion coefficient normally use one or more empirical expressions. There are no real macroscopic models aimed at predicting water diffusion, mainly due to its microscopic nature. The only models used are those which are required for analyzing the experimental data. While there are no models to examine, some discussion is required on the different diffusion coefficients which are used. As mentioned in section 3.2.2.1, there are various driving forces that can be used for the gradient-flux equation, each with their own diffusion coefficient.

In the most general case, one uses the chemical potential as the driving force [91]

$$
\mathbf{N}_{\mathrm{w}}=-\frac{c_{\mathrm{w}} D_{\mu_{\mathrm{w}}}}{R T\left(1-x_{\mathrm{w}}\right)} \nabla \mu_{\mathrm{w}}=-\alpha_{\mathrm{w}} \nabla \mu_{\mathrm{w}}
$$

where $D_{\mu_{\mathrm{w}}}$ is the water diffusion coefficient in the membrane related to a chemical-potential driving force or the thermodynamic diffusion coefficient. The expression may or may not contain the mole fraction term in the denominator depending on how the experimental data were interpreted. While this equation and coefficient can be used as is, if one wants to use something like Fick's law (equation 10) or the dilute-solution-theory water equation (equation 11), the chemical diffusion coefficient can be related to the water-content driving force

$$
\mathbf{N}_{\mathrm{w}}=-c_{\mathrm{w}} D_{\mu_{\mathrm{w}}} \frac{\mathrm{d}\left(\ln a_{\mathrm{w}}\right)}{\mathrm{d} \lambda} \nabla \lambda=-D_{\lambda} \nabla \lambda
$$

where the derivative of activity term is a thermodynamic factor or correction due to nonunity activity coefficients [126]. To determine the factor, differentiation is performed using either water-uptake expressions or models (see section 4.1). As pointed out in the literature [10, 182], at low water contents $\left(\lambda \approx 3\right.$ ) there is peak in the value of $D_{\lambda}$ (due to an inflection in the isotherm; see, for example, Figure 4) which depends highly on the way in which the 
thermodynamic factor is calculated. This is one reason why using $D_{\mu_{\mathrm{w}}}$ and a chemical-potential driving force may be preferable. Finally, one can use a concentration of water driving force, resulting in

$$
\mathbf{N}_{\mathrm{w}}=-\frac{\lambda \bar{V}_{\mathrm{m}}}{\left(\bar{V}_{\mathrm{m}}+\lambda \bar{V}_{\mathrm{w}}\right)^{2}} D_{\lambda} \nabla c_{\mathrm{w}}=-D_{\mathrm{w}} \nabla c_{\mathrm{w}}
$$

where swelling is considered (i.e., an expression akin to equation 47 was used for the concentration). The above equations define the various diffusion coefficients in terms of each other in order to help the reader see their interchangeability and relation to the various modeling driving forces. Only a single diffusion coefficient is necessary for modeling water movement due to its gradient flux.

In terms of semiempirical justification and values, the diffusion coefficient inside the membrane is typically smaller than that of pure water and with a slightly higher activation energy attributed to the confined geometry and strong interactions between water and the membrane. Furthermore, the diffusion coefficient, $D_{\mu_{\mathrm{w}}}$, is typically about a linear function of water content $[90,91,183]$, in agreement with the membrane physical picture.

\section{Special topics}

This section discusses some special aspects, topics, and models related to the membrane. These issues are worth mentioning in terms of macroscopic membrane modeling but have not been discussed above. The first topic examines the issues related to membrane in the catalyst layer with a focus on the reaction site. The second topic relates modeling efforts to model electrolytes and impurity ions in the membrane. The final two topics focus on membrane durability, including transient models, and DMFCs, respectively. 


\subsection{Membrane in the catalyst layers}

Up until now the focus of the chapter has been on the membrane as a separator. In this section, some comments are made about the treatment of the membrane in the catalyst layer; for a more detailed discussion on catalyst-layer modeling the reader is referred to another review [1]. Ionomer is placed in catalyst layers in order to provide a conduction path for the protons and extend the reaction zone. In essence, it is used to make the layer into a porous electrode. These porous electrodes are the most complicated of all the fuel-cell-sandwich regions since they contain all of the various phases as well as supported electrocatalyst. The resistance of the membrane in the catalyst layers can be appreciable and has an effect on the reaction-rate distribution. This section is divided into two main themes based on the use of an embedded macrohomogeneous model [1] for the catalyst layer. The first focuses on how to modify the governing equations for the membrane in a catalyst layer, and the second examines the nature of the membrane and the reaction site in terms of modeling. The former can be considered changing the membrane equations on a macroscopic length scale (i.e., that of the catalyst layer), and the latter on a more microscopic, local one (i.e., the reaction site).

\subsubsection{Governing equations and properties (layer length scale)}

Since membrane exists throughout the catalyst layer, the modeling treatments and equations discussed in the previous sections of this chapter remain valid, although they may have to be altered slightly. All of the porous-electrode-type models incorporate the following changes [1]. 
In terms of properties, the only difference is that since the membrane does not occupy the entire volume of the layer, something like a Bruggeman expression is required to alter the transport-property values [184-187]

$$
Y=Y_{\mathrm{o}} \frac{\varepsilon_{\mathrm{m}}}{\tau_{\mathrm{m}}}=Y_{\mathrm{o}} \varepsilon_{\mathrm{m}}^{1.5}
$$

where $Y$ stands for a property such as conductivity, the o denotes a bulk property value, and $\varepsilon_{\mathrm{m}}$ and $\tau_{\mathrm{m}}$ are the membrane-phase volume fraction and tortuousity, respectively.

In terms of the governing equations, the appropriate source terms must be added to the conservation equations. The transport equations and constitutive relations remain the same except for the use of modified property expressions. For the current balance (equation 5), the transfer current between the membrane and electronically conducting solid must be included

$$
\nabla \cdot \mathbf{i}_{\mathrm{m}}=-\nabla \cdot \mathbf{i}_{\mathrm{s}}=a_{0} i_{h}
$$

where $\nabla \cdot \mathbf{i}_{\mathrm{m}}$ represents the total anodic rate of the electrochemical reaction per unit volume of electrode, $\mathrm{m}$ and $\mathrm{s}$ denote the membrane and solid phase, respectively, $a_{0}$ is the specific interfacial reaction area, and $i_{h}$ is the transfer current for reaction $h$. For the hydrogen oxidation reaction (HOR) and the oxygen reduction reaction (ORR), the transfer current expressions are [1]

$$
i_{\text {HOR }}=i_{0_{\text {HOR }}}\left[\frac{p_{\mathrm{H}_{2}}}{p_{\mathrm{H}_{2}}^{\text {ref }}} \exp \left(\frac{\alpha_{a} F}{R T}\left(\eta_{\text {HOR }}\right)\right)-\exp \left(\frac{-\alpha_{c} F}{R T}\left(\eta_{\text {HOR }}\right)\right)\right]
$$

and

$$
i_{\mathrm{ORR}}=-i_{\mathrm{ORR}_{\mathrm{ORR}}}\left(\frac{p_{\mathrm{O}_{2}}}{p_{\mathrm{O}_{2}}^{\text {ref }}}\right) \exp \left(\frac{-\alpha_{c} F}{R T}\left(\eta_{\mathrm{ORR}}\right)\right)
$$

respectively, where $\alpha_{a}$ and $\alpha_{c}$ are the anodic and cathodic transfer coefficients, respectively, ref denotes the value at its reference conditions, and $\eta_{h}$ is the overpotential for reaction $h$. 
For the mass balance (equation 2), the appropriate source terms must be included. Because it is assumed that the electrochemical reactions occur in the membrane (see section 5.1.2), the mass-balance expression for species $i$ in the membrane becomes

$$
\frac{\partial c_{i}}{\partial t}=-\nabla \cdot \mathbf{N}_{i}+S_{i}+R_{i}
$$

where $R_{i}$ is the transfer of species $i$ into or out of the membrane through mass-transfer or equilibrium expressions as discussed in sections 4.1 and 5.1.2, and $S_{i}$ is the generation/consumption term of species $i$ that is given by Faraday's law

$$
N_{i}=\sum_{h} s_{i, h} \frac{i_{h}}{n_{h} F}
$$

where $s_{i, h}$ is the stoichiometric coefficient of species $i$ participating in reaction $h(-1$ for hydrogen in the HOR, and -1 and 2 for oxygen and water in the ORR, respectively), and $n_{h}$ is the number of electrons transferred in reaction $h$ ( 2 for HOR and 4 for ORR).

For the energy balance (equation 23), the appropriate source terms must be added, yielding [1]

$$
\rho \hat{C}_{p}\left(\frac{\partial T}{\partial t}+\mathbf{v} \cdot \nabla T\right)=-\nabla \cdot \mathbf{q}+\sum_{i} \bar{H}_{i} \nabla \cdot \mathbf{J}_{i}+\sum_{h} a_{0} i_{h}\left(\eta_{s_{h}}+\Pi_{h}\right)+\sum_{g} \Delta H_{g} R_{g}
$$

where the last term on the right includes enthalpy generation/consumption due to reactions (e.g., evaporation) and the second to last term that due to the electrochemical reactions. The latter contains expressions for both the reversible and irreversible heat generation, as derived by Newman and coworkers $[59,128,129,188]$. The irreversible heat generation is represented by the surface overpotential, and the reversible by the Peltier coefficient [189]. While almost all of the catalyst-layer models use the mass- and current-balance equations, only a few $[37,44,47,71$, $89,105,111,190]$ use the above energy equation. 


\subsubsection{Reaction-site models (local length scale)}

While the previous section discusses the changes to the governing equations on the layer length scale, it did not discuss the local length scale or where the reaction site is. As mentioned, it is assumed that the reactions occur within the membrane phase of the catalyst layers. However, there are various modeling methodologies and pictures for how this occurs [1]. To determine the correct modeling equations and how the membrane is involved, an analysis is done where three models are chosen and fit to experimental data. The three models are 1-D fuel-cell sandwich models that are identical except for their rate expressions $[172,191]$, which are given in terms of the ORR below for brevity, and can easily be adapted for the HOR.

In the first model [11, 12, 37, 53, 89, 102, 111, 172, 192, 193], denoted PE, just the porouselectrode equations are used (as given in the previous section), and the reaction site is assumed to be just the catalyst interface.

In the second model $[46,50,124]$, denoted PEF, the porous-electrode equations are used, and the reaction site is assumed to be an interface covered by a thin membrane film. Hence, the current balance for the ORR can be expressed as

$$
\nabla \cdot \mathbf{i}_{\mathrm{m}}=4 F c_{\mathrm{O}_{2}}^{\text {bulk }}\left(\frac{1}{\frac{\delta_{\text {film }}}{A D_{\mathrm{O}_{2}, \text { film }}}+\frac{1}{k^{\prime}}}\right)
$$

where bulk signifies the concentration outside the film, the reference concentration is that in the membrane in equilibrium with the reference pressure, $\delta_{\text {film }}$ and $A$ are the thickness and specific external surface area of the film, respectively, and 


$$
k^{\prime}=\frac{a_{0} i_{0_{\mathrm{ORR}}}}{4 F c_{\mathrm{O}_{2}}^{\mathrm{ref}}} \exp \left(-\frac{\alpha_{c} F}{R T}\left(\eta_{\mathrm{ORR}}\right)\right)
$$

In the third model [56, 57, 76, 103, 194-199], denoted PEA, the porous-electrode equations are used and the reaction site is assumed to be a spherical agglomerate composed of supported catalyst, membrane, and possible gas micropores. For this model, the current balance for the ORR is given by

$$
\nabla \cdot \mathbf{i}_{\mathrm{m}}=a_{0} i_{\mathrm{ORR}} E
$$

where $E$ is the effectiveness factor, which for a first-order reaction can be written as $[126,200]$

$$
E=\frac{1}{3 \phi^{2}}(3 \phi \operatorname{coth}(3 \phi)-1)
$$

where $\phi$ is the Thiele modulus for the system [201]

$$
\phi=\frac{R_{\mathrm{agg}}}{3} \sqrt{\frac{k^{\prime}}{D_{\mathrm{O}_{2}, \mathrm{ggg}}^{\mathrm{e}}}}
$$

where $R_{\text {agg }}$ is the radius of the agglomerate. In the above derivation, the agglomerate was assumed to be equipotential. If this assumption is not used or if the reaction is not first order, than an analytic expression is not obtainable; a separate numerical model must be used.

The three models were fit to various experimental polarization curves, where the PE model had 2 fitting parameters and the PEF and PEA models each had three, all of which were similar in their effect. The fits are shown in Figure 13. As is readily apparent, the PEA model fits the experimental data much better than the others, in agreement with other literature comparisons that used simpler fuel-cell and membrane models [204-206]. For the three cases, the PEF model mainly follows the PE model in terms of fitting the curve, with the possible exception of case (c) where the PEF model results in a better fit. Overall however, the PE and PEF models result in a pronounced "knee" in the mass-transfer portion of the curve. Thus, one can surmise that the 
added fitting parameter and diffusion resistance provided by the PEF model has only a minimal impact. Therefore, a single barrier to oxygen transport is not limiting, and one needs combined reaction and diffusion and significant interaction with the membrane. Thus, the agglomerate model is the best to use for the reaction site of membrane, electrocatalyst, and gas (either dissolved or free).

The reason why the PEA model fits best and an effectiveness factor is required is that it allows for a more gradual decrease in the reaction rate or transfer current as a function of decreasing potential, as shown in Figure 14. This is because the oxygen partial pressure decreases gradually, due to the variable reaction penetration depth into the agglomerate because of the consideration of simultaneous reaction and diffusion. Further examination of the figure shows that, at low current densities, the effectiveness factor for the anode is lower than for the cathode because of the facile kinetics of the HOR, but is higher at higher current densities due to the higher hydrogen concentration in the anode catalyst layer and faster hydrogen diffusion in the agglomerate. However, it is the cathode catalyst layer and its effectiveness factor that are limiting in all the cases examined.

In summary, the membrane model for the layer length scale is basically the same as for the membrane itself, except that the properties need to be adjusted and source terms have to be added to the conservation equations. For the local length scale, the reaction site is best treated using an agglomerate model because it allows for an effectiveness factor that changes gradually with potential versus that of a constant for a bare or film-covered reaction site. 


\subsection{Impurity ion and electrolyte effects}

The existence of other ions in the membrane besides protons and sulfonic acid sites (e.g., platinum, iron, sodium, etc.) complicates the transport and modeling of the membrane. In fact, the other ions often act as poisons which decrease fuel-cell performance, although additional protons from acids may increase the conductivity [163]. The ions can come from a variety of sources including end or bipolar plates, corrosion reactions, catalyst dissolution (see section 5.3), and contaminants left over from manufacturing. To model such effects requires the incorporation of additional phenomena into the governing equations. Because these effects are mainly of secondary importance in fuel cells, the developed models are aimed at other applications of the membranes such as in separation processes and the chlor-alkali industry. For these reasons and because they are more microscopic in approach, the model treatments are discussed only briefly below.

There are three main electrolyte issues for the membrane, which can be categorized as effects due to cation exchange, the presence of electrolyte in the reservoir next to the membrane, and the presence of electrolyte inside the membrane pores. PFSA membranes readily undergo cation exchange, for example, like sodium ions will replace the protons associated with the sulfonic acid sites. Such a replacement has obvious implications in terms of changing the transport properties of the membrane [207], with the decrease in proton conduction the most expected and significant due to the inability to form hydrogen bonds and utilize the Grotthuss mechanism. More than any other group, Okada and coworkers have modeled such effects [143, 208-210]. They analytically solve the dilute-solution equations described in section 3, but incorporate the changing transport properties, usually through some kind of linear average. An example of their simulation results is shown in Figure 15. 
The figure demonstrates how having $10 \%$ contamination of sodium ions on the cathode side of the membrane greatly influences the water management and ohmic resistance through the membrane. These effects are especially significant at high current densities. One can see that the water content decreases and the net water flux is higher, resulting in significant dehydration of the membrane, which is apparent in the much higher resistance values. The net water flux is higher due to a much larger electro-osmotic flux through the membrane in its sodium rather than hydrogen or acid form. This effect and the changes in the other transport properties cause the resistance of the membrane and the net water flux to be nonlinear functions of the current density, as one might have expected. Overall, the results clearly demonstrate the significant problems and changes associated with having cation impurities and part of the membrane cation exchanged.

While cations may exchange with the protons in the membrane, they can also exist in the hydrophilic-phase within the membrane as neutral combinations with anions such as chloride, even though one expects some anion exclusions and some cation exchange due to the presence of the anionic sulfonic acid sites. Thus, something like platinum in the membrane should decrease the membrane's conductivity in the same fashion as described above. These impurity ions may also cause other physicochemical changes such as different microstructure due to crosslinking, ion-pair formation, etc. [208]. They will also alter the uptake properties of the membranes.

To calculate the distribution of ions in the membrane in contact with a reservoir, one needs to consider chemical equilibrium among the various species in the membrane and their counterparts in the external reservoir. To do this, one needs to calculate the concentration distribution of each ion inside of the pore. This distribution can be written as a modified Boltzmann distribution [4, 211-214] 


$$
c_{i}(r)=c_{i}^{\mathrm{ext}} \exp \left[-\frac{z_{i} F \Phi(r)}{R T}-\frac{A_{i}}{R T}\left(\frac{\varepsilon_{0}}{\varepsilon_{r}(r)}-\frac{\varepsilon_{0}}{\varepsilon_{r}^{\mathrm{ext}}}\right)\right]
$$

where $r$ is the radial position of the pore, ext denotes the external reservoir, $A_{i}$ is an ion hydration constant of species $i$ (see reference [213] for values), and $\varepsilon_{r} / \varepsilon_{0}$ is the dielectric constant of the medium, where $\varepsilon_{0}$ is the permittivity of free space. In the above expression, the first term represents electrostatic attraction/repulsions of ions, and the second relates the effects of a changing dielectric medium. If the dielectric constant is uniform and the same as the reservoir, then the expression becomes a normal Boltzmann distribution.

In order to calculate the distribution, the dielectric-constant and potential distributions must be known. The dielectric-constant distribution can be determined using Booth's equation [215]

$$
\frac{\varepsilon(r)}{\varepsilon_{0}}=n^{2}+\frac{3\left(\frac{\varepsilon_{r}^{\mathrm{ext}}}{\varepsilon_{0}}-n^{2}\right)}{\zeta \nabla \Phi(r)}\left[\operatorname{coth}(\zeta \nabla \Phi(r))-\frac{1}{\zeta \nabla \Phi(r)}\right]
$$

where $n$ is the refractive index of the solution and $\zeta$ is

$$
\zeta=\left(\frac{5 \eta}{2 k T}\right)\left(n^{2}+2\right)
$$

where $k$ is Boltzmann's constant and $\eta$ is the dipole moment of the solvent molecule. Finally, the potential distribution can be calculated by solving Poisson's equation [59]

$$
\nabla \cdot(\varepsilon(r) \nabla \Phi(r))=-\rho_{e}=-F \sum_{i=1}^{m} z_{i} c_{i}(r)
$$

where $\rho_{e}$ is the charge density and the summation is over all charged species. Solving equations 70,71 , and 73 simultaneously yields the concentration distributions of the various ions and the potential distribution within the pore. 
The potential and dielectric-constant distributions for various pore sizes as calculated by Pintauro and Verbrugge [214] are shown in Figure 16. The figure clearly demonstrates that the dielectric constant and potential are not uniform within a significant distance of the pore wall, in agreement with other analyses $[4,213]$. The potential deviates due to both dielectric-constant variation and especially hydration effects as also shown by Pintauro and Verbrugge. As can be seen in the figure, pore sizes around $2 \mathrm{~nm}$ and below do not have any regions of uniform potential or dielectric constant. This size correlates with partially hydrated pore sizes (i.e., when $\lambda<7$ ), and one can draw the inference that bulk-like water forms when the potential and dielectric-constant distributions flatten and become equal to that of water, as mentioned in section 2.3. In terms of ion distributions and uptake, the overall partitioning of salts is shown to be dependent on the monovalent rather than the divalent cation, with the larger monovalent cations being more selective than the smaller ones $[212,213]$. In all, the above approach allows one to predict ion partitioning by these membranes, which is of special interest in separation applications. Furthermore, the potential-distribution calculation is also a key component of most microscopic models [4], including the ones for conductivity and electro-osmotic flow.

The last topic of discussion in this section is that of transport of electrolytes through the membrane. This transport is seen in dissolution studies where one finds platinum and alloy catalyst movement through the membrane after operation [130, 216-219]. To model such effects, the same basic approaches and equations as described in section 3 have been used [92, 216, 220-222].

The major differences are that not all of the current is carried by protons and the system is no longer only three components. The major modification of the equations involves a more general definition of the current density 


$$
\mathbf{i}=F \sum_{i} z_{i} \mathbf{N}_{i}
$$

This also means that a more general expression can be derived for the potential gradient

$$
\nabla \Phi=-\frac{\mathbf{i}}{\kappa}-\frac{F}{\kappa} \sum_{i} z_{i} D_{i} \nabla c_{i}
$$

where the conductivity can be defined as

$$
\kappa=F^{2} \sum_{i} z_{i}^{2} u_{i} c_{i}
$$

In addition to the above equations, material balances need to be applied for each species (equation 2), the current and energy balances remain the same (equations 5 and 23, respectively), and either a Nernst-Plank equation (equation 8) or a multicomponent transport one (e.g., equation 15) depending on the approach taken. Finally, either electroneutrality (equation 4) can be assumed or, for the more microscopic models, Poisson's equation (equation 73) along with equations 70 and 71 can be incorporated into the governing set of equations.

Due to the presence of more species, more properties are required. These can take the form of mobilities, diffusion coefficients, conductivity, and transference numbers [59]

$$
t_{j}=\frac{z_{j}^{2} u_{j} c_{j}}{\sum_{i} z_{i}^{2} u_{i} c_{i}}
$$

which is the fraction of current carried by the ion $j$ in the absence of concentration gradients. Without other ions, this value is 1 for protons, and it can be used as an indication of the current transport efficiency. As a side note, the electro-osmotic coefficient is basically the transference number of water. For the multicomponent systems, concentrated solution theory, which is given in terms of binary interaction parameters that can be related to the above experimentally measurable properties, predicts the correct number of transport properties whereas dilution solution does not [59]. In essence, all of the interactions are treated explicitly. Furthermore, to 
be rigorous, one must also consider activity coefficients for neutral combinations and thermodynamic factors (see section 4.3 and reference [59]).

\subsection{Membrane durability and transient modeling}

The most important issue facing PEFCs today is probably that of lifetime and durability. As the heart of the fuel cell, the membrane is a key part in understanding failure. This is especially true in those cases where the membrane is either chemically or physically altered, resulting in pinhole formation and catalyst layer delamination [130,219], or where the cause of failure can be traced to such issues as gas crossover through the membrane [223]. In addition, the poisoning effects described above in terms of cations in the membrane (including platinum) can lead to performance loss [223-225]. In terms of modeling, there has been some work on durability and failure mechanisms, but it is relatively new; understanding failure is one of the main goals of current and future modeling activities.

Any model of failure and durability is inherently a transient one. This is because these effects occur due to fluctuations in operating conditions like potential or during transient operation (e.g., startup and shutdown). In terms of transient membrane modeling, various groups have examined the behavior of the water content of the membrane $[40,72,82,89,108,120,226$, 227]. The governing equations are essentially the same as those in section 3 , although with the time dependent terms considered. They clearly show that operation with dry gases and high stoichiometries leads to cases of unstable operation where the membrane dries out and the fuel cell fails. They have also shown much more instability in operation due to the transients in water management. This last is shown in Figure 17 in terms of the time lag that it takes for a membrane to become fully hydrated after a step change in relative humidity. This lag causes a 
very nonuniform current distribution along the channel not to mention water buildup at the cathode, both of which can lead to instabilities and possible durability issues. It is worth noting that the overall-system-response time constant is the same as that for membrane hydration.

In terms of membrane failure mechanisms, the most modeled is pinhole formation due to membrane degradation by chemical attack of peroxide $[132,223,228]$. The peroxide is generated in small amounts in the oxygen reduction reaction and in greater amounts when oxygen crosses over and reacts via a 2 electron transfer reaction to peroxide on the anode [131, 229]. It is also generated during potential cycling on the cathode where oxide and hydroxide layers form on the platinum catalysts [217]. The generated peroxide attacks the membrane end groups and essentially unzips the polymer [132]. While the qualitative picture is known and improvements have been made in terms of durability, there has been no quantitative model of this effect yet.

Other membrane failure mechanisms include mechanical effects during cycling (especially with humidity and temperature) [130, 219], carbon corrosion [230-232], and cation impurities and movement in the membrane $[130,216-219,224]$. For the first mechanism, there has been no substantial modeling effort as of yet, although there is ongoing work on this topic in the community. For the second topic, Resier et al. [232] put forth a reverse-current decay mechanism in which carbon corrosion occurs due to inadequate fuel quantities and possible crossover during startup and shutdown. For the third topic, it has been suggested that these ions come from platinum dissolution [216-218] at the cathode or iron from the end plates [224] or other sources of impurities.

In terms of modeling, some effort has been done on the dissolution mechanism of platinum $[217,233]$ and on platinum migration using dilute solution equations as mentioned in the 
preceding section [216]. Also mentioned there are issues associated with cation exchanged membranes causing water management problems and changes in transport properties and perhaps membrane microstructure [208]. Of note is the model of Chen et al. [144], which shows the time and required current density for stable operation is increased with impurity ions in the membrane. Finally, in terms of incorporating failure, Jiang and Chu [234] use an empirical membrane deterioration term that limits the maximum water content of the membrane in their model.

\subsection{Direct-methanol fuel cells}

The membranes used for DMFCs are typically the same as those for hydrogen fuel cells (i.e.,

Nafion ${ }^{\circledR}$ ), although they may be thicker and have slightly smaller and more tortuous pores. This is because performance loss due to methanol crossover is more important than membrane conductivity, especially since the membrane remains fully hydrated.

Since the membranes are basically identical, the same can be said of the membrane models [121, 174, 235-243]. A benefit of DMFCs is that liquid water exists on both sides of the membrane (in most cases at high enough current densities), and thus it remains liquidequilibrated throughout. This means that the membrane model can be simplified, especially with respect to conductivity. However, water flow becomes more complicated due to methanol crossover. Unlike gas crossover in hydrogen fuel cells, methanol can exist in appreciable quantities due to its miscibility with water. This means that it cannot be taken as a minor component, and the appropriate transport equations must be used.

The most common approach for modeling water and methanol movement in the membrane is by using the convective diffusion equation (equation 31) for both water and methanol, where the 
bulk velocity is given by the Schlögl equation (equation 18). A slightly more complicated approach is to use a convective diffusion equation for methanol that also includes an electroosmotic term with a different coefficient than that of water. In essence, both of these are basically dilute-solution approaches. While they will yield general trends and answers, it is problematic in terms of determining the correct property expressions like diffusion and electroosmotic coefficients that should be used in the equations. Finally, the uniform membrane hydration along with a simple methanol diffusion equation allow for analytic models and for the equations to be solved exactly for specific cases, such as that of constant and equimolar feeds $[121,241,242]$.

A more realistic and complicated approach is to use a concentrated-solution-theory analog [174]. The equations used are derived the same as in section 3.2.2.2, except that there is the additional methanol equation along with its coefficients and cross coefficients, all of which have to be determined. The concentrated-solution-theory approach also accounts for the fact that uptake of the membrane both in terms of water and methanol depends on the concentration of the outside reservoir. While three-phase diagrams exist for this system (for example, see reference [148]), there is a minimal amount of modeling to predict the uptake. The best and most rigorous treatment for water uptake is that of Meyers and Newman $[18,174]$ using their chemical model. Starting from a single Gibbs function for the membrane [158], they derive expressions for the chemical potential of water and methanol in the membrane, and equate those to the chemical potentials of water and methanol in the reservoir. This is essentially the same treatment as their chemical model for uptake from water vapor (see section 4.1), except that now it is from a liquid water/methanol reservoir. Such a modification requires careful consideration of the appropriate reference states and determination of activity coefficients. Overall, modeling a DMFC 
membrane is very similar to modeling a PEFC membrane (the underlying physics are the same), except now there is liquid water throughout although it is not pure (the methanol must be accounted for with its own governing equation and interactions).

\section{Summary}

In this chapter, we have examined the different models for polymer-electrolyte membranes used in fuel cells operating with hydrogen. The major focus has been on the governing equations and approaches for transport of the various species within the membrane. This included a discussion of the various governing equations and property values such as membrane water content. Concentrated solution theory was shown to be the most rigorous, and the approach that can best address the full range of membrane water contents and conditions. Some related special topics of interest including membrane treatment in catalyst layers and durability issues were also discussed.

Full membrane models were not directly compared to each other; instead they were broken down into their constitutive parts. The reason for this is that validation of the models is usually accomplished by comparison of simulation to experimental polarization data (e.g., Figure 3), which necessitates at least a 1-D sandwich model of the entire cell. Furthermore, in fitting this data, the models not only vary in their complexity and treatments, but also in their number and kind of fitting parameters. This is one reason why it is hard to justify one approach over another by just looking at the modeling results. In general, it seems reasonable that the more complex models, which are based on physical arguments and do not contain many fitting parameters, are perhaps closest to reality. Of course, this assumes that they fit the experimental data and observations under a variety of operating conditions and parameter values. 
This review has highlighted the important effects that should be modeled. These include a robust water-uptake model, a combination model for transport that remains valid for both vaporand liquid-equilibrated membranes, and inclusion of membrane swelling, among others. For any model, a balance must be struck between the complexity required to describe the physical reality and the additional costs of such complexity. In other words, while more complex models more accurately describe the physics of the transport processes (e.g., detailed pore models of potential distributions for proton conduction), they are more computationally costly and may contain many fitting parameters. Overall, membrane modeling and understanding has progressed substantially in the last few years, and the research community is now closing in on good descriptions of the underlying transport phenomena, microstructure, etc. Such treatments mean that simulations can now begin to optimize and perhaps even aid in designing fuel-cell membranes. Hopefully, this review has shown and broken down for the reader the complexities and approaches undertaken in modeling polymer-electrolyte membranes.

\section{Acknowledgements}

We would first like to thank the financial support of the the Assistant Secretary for Energy Efficiency and Renewable Energy, Office of Hydrogen, Fuel Cell, and Infrastructure Technologies, of the U. S. Department of Energy under contract number DE-AC02-05CH11231. We would also like to thank Trung V. Nguyen and Tim Zhao for inviting us to write this chapter. 


\section{Notation}

\section{Roman}

$a_{i} \quad=$ activity of species $i$

$a_{0} \quad=$ interfacial area of reaction between the solid and membrane phases $/ \mathrm{cm}^{-1}$

$A \quad=$ specific external surface area $/ \mathrm{cm}^{-1}$

$A_{i} \quad=$ ion hydration constant of species $i / \mathrm{J} \mathrm{mol}^{-1}$

$b \quad=$ Tafel slope $/ \mathrm{V}$

$c_{i} \quad=$ interstitial concentration of species $i / \mathrm{mol} \mathrm{cm}^{-3}$

$c_{\mathrm{T}}=$ total solution concentration $/ \mathrm{mol} \mathrm{cm}{ }^{-3}$

$\hat{C}_{p}=$ membrane system heat capacity $/ \mathrm{J} \mathrm{g}^{-1} \mathrm{~K}^{-1}$

$\mathbf{d}_{i} \quad=$ driving force per unit volume acting on species $i / \mathrm{J} \mathrm{cm}^{-4}$

$D_{i} \quad=$ Fickian diffusion coefficient of species $i / \mathrm{cm}^{2} \mathrm{~s}^{-1}$

$D_{i, j}=$ diffusion coefficient of $i$ in $j / \mathrm{cm}^{2} \mathrm{~s}^{-1}$

$D_{\mu_{\mathrm{w}}}=$ diffusion coefficient of water related to a chemical potential driving force $/ \mathrm{cm}^{2} \mathrm{~s}^{-1}$

$D_{\lambda} \quad=$ diffusion coefficient of water related to a water content driving force $/ \mathrm{cm}^{2} \mathrm{~s}^{-1}$

$E \quad=$ effectiveness factor, defined by equation 68

$E_{W} \quad=$ membrane equivalent weight, g/equiv

$E_{0} \quad=$ Young's modulus reference value $/ \mathrm{N} \mathrm{cm}^{-2}$

$f \quad=$ water volume fraction in the membrane

$F \quad=$ Faraday's constant $/ 96487 \mathrm{C} \mathrm{equiv}^{-1}$

$G=$ membrane shear modulus $/ \mathrm{N} \mathrm{cm}^{-2}$

$\Delta G=$ Gibbs free energy of reaction $/ \mathrm{J} \mathrm{mol}^{-1}$

$\bar{H}_{i} \quad=$ partial molar enthalpy of species $i / \mathrm{J} \mathrm{mol}^{-1}$

$\Delta H_{l}=$ heat or enthalpy of reaction $l, \mathrm{~J} \mathrm{~mol}^{-1}$

$i=$ superficial current density through the membrane $/ \mathrm{A} \mathrm{cm}^{-2}$

$\mathrm{i} \quad=$ superficial current density $/ \mathrm{A} \mathrm{cm}^{-2}$

$i_{h}=$ transfer current density of reaction $h$ per interfacial area $/ \mathrm{A} \mathrm{cm}^{-2}$

$i_{0_{h}} \quad=$ exchange current density for reaction $h / \mathrm{A} \mathrm{cm}^{-2}$

$i_{\lim }=$ limiting current density $/ \mathrm{A} \mathrm{cm}^{-2}$

$\mathbf{J}_{i}=$ flux density of species $i$ relative the mass-average velocity $/ \mathrm{mol} \mathrm{cm}^{-2} \mathrm{~s}^{-1}$

$k=$ Boltzmann's constant $/ 1.38065 \times 10^{-23} \mathrm{~J} \mathrm{~K}^{-1}$

$k=$ effective permeability $/ \mathrm{cm}^{2}$

$k_{h}^{\prime} \quad=$ reaction-rate portion of the Thiele modulus $/ \mathrm{mol} \mathrm{bar}^{-1} \mathrm{~cm}^{-3} \mathrm{~s}^{-1}$

$k_{T} \quad=$ effective thermal conductivity $/ \mathrm{J} \mathrm{cm}^{-2} \mathrm{~K}^{-1}$

$k_{\Phi}=$ electrokinetic permeability $/ \mathrm{cm}^{2}$

$K_{i, j}=$ frictional coefficient of interaction between species $i$ and $j / \mathrm{J} \mathrm{s} \mathrm{cm}^{-5}$ 


$$
\begin{aligned}
& K_{l} \quad=\text { equilibrium coefficient for reaction } l \\
& l=\text { membrane thickness } / \mathrm{cm} \\
& L_{i, j} \quad=\text { inverted frictional coefficient of interaction between species } i \text { and } j / \mathrm{cm}^{5} \mathrm{~J}^{-1} \mathrm{~s}^{-1} \\
& M_{i} \quad=\text { molecular weight of species } i / \mathrm{g} \mathrm{mol}^{-1} \\
& n \quad=\text { solution refractive index } \\
& n \quad=\text { number of electrons transferred in a reaction } \\
& N \quad=\text { number of species } \\
& \mathbf{N}_{i} \quad=\text { superficial flux density of species } i / \mathrm{mol} \mathrm{cm}^{-2} \mathrm{~s}^{-1} \\
& p=\text { total thermodynamic pressure } / \mathrm{N} \mathrm{cm}^{-2} \\
& p_{i} \quad=\text { partial pressure of species } i / \mathrm{N} \mathrm{cm}^{-2} \\
& p_{k}=\text { total pressure of phase } k / \mathrm{N} \mathrm{cm}^{-2} \\
& p_{\mathrm{w}}^{\text {vap }}=\text { vapor pressure of water } / \mathrm{N} \mathrm{cm}^{-2} \\
& \mathbf{q}=\text { superficial heat flux } / \mathrm{J} \mathrm{cm}^{-2} \mathrm{~s}^{-1} \\
& r=\text { membrane pore radius } / \mathrm{cm} \\
& r_{\mathrm{c}} \quad=\text { critical pore radius } / \mu \mathrm{m} \\
& R \quad=\text { ideal-gas constant } / 8.3143 \mathrm{~J} \mathrm{~mol}^{-1} \mathrm{~K}^{-1} \\
& R_{\text {agg }}=\text { agglomerate radius } / \mathrm{cm} \\
& R_{i} \quad=\text { rate of transfer of species } i \text { to/from the membrane } / \mathrm{mol} \mathrm{cm}^{-3} \mathrm{~s}^{-1} \\
& R_{\Omega} \quad=\text { total constant ohmic resistance } / \Omega \mathrm{cm}^{2} \\
& s_{i, h} \quad=\text { stoichiometric coefficient of species } i \text { participating in reaction } h \\
& S \quad=\text { fraction of expanded channels } \\
& S_{i} \quad=\text { rate of generation/consumption of species } i / \mathrm{mol} \mathrm{cm}^{-3} \mathrm{~s}^{-1} \\
& \bar{S}_{i} \quad=\text { molar entropy of species } i / \mathrm{J} \mathrm{mol}^{-1} \mathrm{~K}^{-1} \\
& t=\text { time } / \mathrm{s} \\
& t_{j} \quad=\text { transference number of species } j \\
& T=\text { absolute temperature } / \mathrm{K} \\
& u_{i} \quad=\text { mobility of species } i / \mathrm{cm}^{2} \mathrm{~mol} \mathrm{~J}^{-1} \mathrm{~s}^{-1} \\
& U^{\theta} \quad=\text { standard cell potential } / \mathrm{V} \\
& \mathbf{v} \quad=\text { superficial solution velocity } / \mathrm{cm} \mathrm{s}^{-1} \\
& \mathbf{v}_{i} \quad=\text { superficial velocity of species } i / \mathrm{cm} \mathrm{s}^{-1} \\
& V \quad=\text { cell potential, } \mathrm{V} \\
& \bar{V}_{i} \quad=\text { (partial) molar volume of species } i / \mathrm{cm}^{3} \mathrm{~mol}^{-1} \\
& x_{i} \quad=\text { mole fraction of species } i \\
& Y \quad=\text { generic property such as conductivity or transport coefficient } \\
& z \quad=\text { through-plane (thickness) distance of the membrane } / \mathrm{cm} \\
& z^{\prime} \quad=\text { modified membrane thickness coordinate to account for swelling } / \mathrm{cm} \\
& z_{i} \quad=\text { valence or charge number of species } i
\end{aligned}
$$


$\underline{\text { Greek }}$

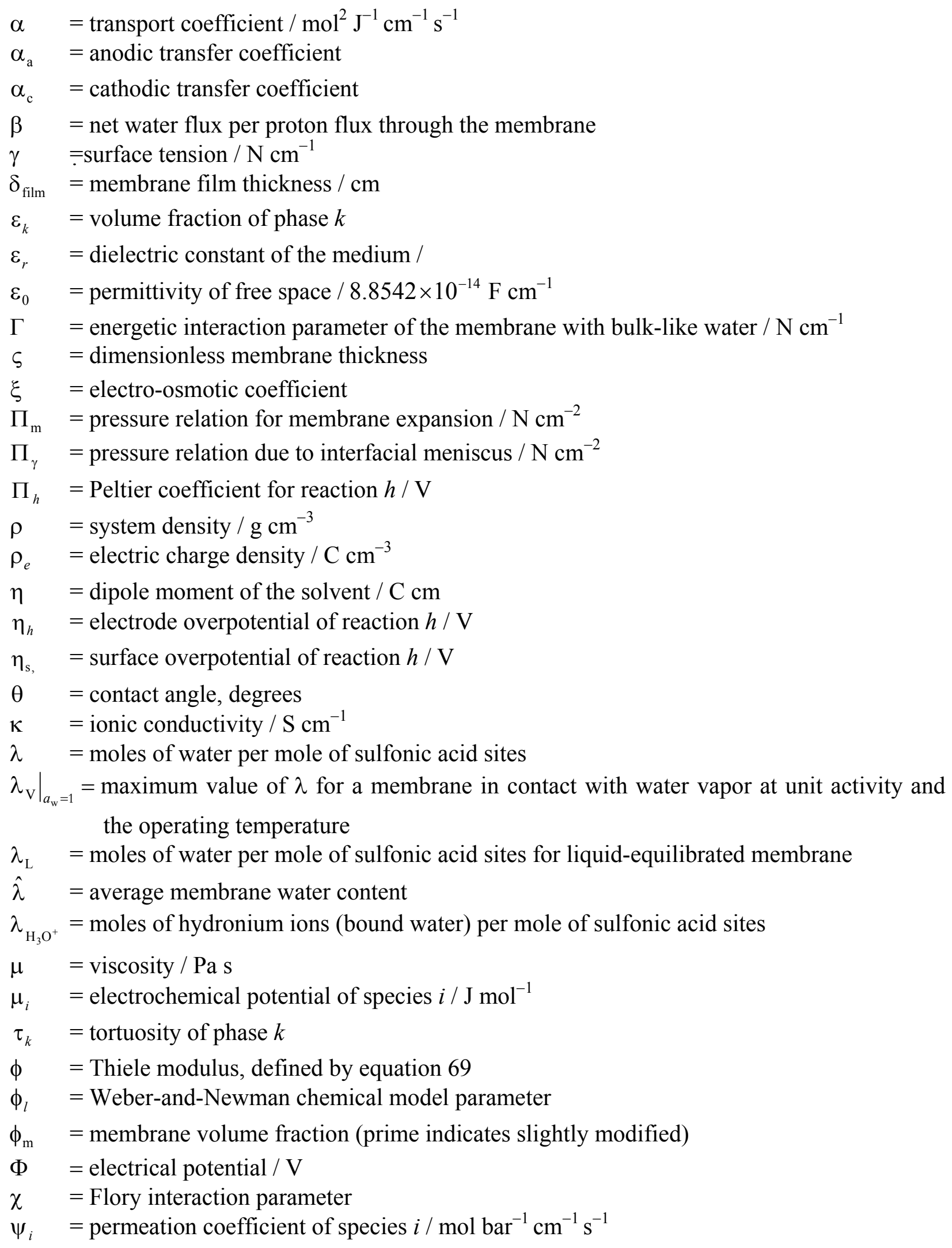




\section{$\underline{\text { Subscripts/Superscripts }}$}

$$
\begin{array}{ll}
\text { e } & =\text { effective transport property } \\
\text { ext } & =\text { external reservoir conditions } \\
f & =\text { fixed ionic site } \\
\mathrm{H}^{+} & =\text {proton } \\
\mathrm{HOR} & =\text { hydrogen oxidation reaction } \\
\mathrm{m} & =\text { membrane } \\
\mathrm{O} & =\text { initial or reference value } \\
\mathrm{ORR} & =\text { oxygen reduction reaction } \\
\mathrm{ref} & =\text { parameter evaluated at the reference conditions } \\
\mathrm{S} & =\text { solid phase } \\
\mathrm{W} & =\text { water }
\end{array}
$$




\section{Literature cited}

1. A. Z. Weber, J. Newman, Chem. Rev., 104 (2004) 4679.

2. C. Y. Wang, Chem. Rev., 104 (2004) 4727.

3. J. Fimrite, H. Struchtrup, N. Djilali, J. Electrochem. Soc., 152 (2005) A1804.

4. K. D. Kreuer, S. J. Paddison, E. Spohr, M. Schuster, Chem. Rev., 104 (2004) 4637.

5. B. Carnes, N. Djilali, J. Power Sources, 144 (2005) 83.

6. M. V. Williams, H. R. Kunz, J. M. Fenton, J. Electrochem. Soc., 152 (2005) A635.

7. A. A. Kulikovsky, Electrochem. Commun., 4 (2002) 845.

8. H. A. Liebhafsky, E. J. Cairns, W. T. Grubb, L. W. Niedrach, in: G. J. Young, H. R. Linden (Ed.), Fuel Cell Systems, 47, American Chemical Society, Washington, D.C., 1965, p. 116.

9. T. F. Fuller, J. Newman, J. Electrochem. Soc., 140 (1993) 1218.

10. T. E. Springer, T. A. Zawodzinski, S. Gottesfeld, J. Electrochem. Soc., 138 (1991) 2334.

11. D. M. Bernardi, M. W. Verbrugge, AIChE J., 37 (1991) 1151.

12. D. M. Bernardi, M. W. Verbrugge, J. Electrochem. Soc., 139 (1992) 2477.

13. K. D. Kreuer, Solid State Ionics, 97 (1997) 1.

14. A. Z. Weber, J. Newman, J. Electrochem. Soc., 150 (2003) A1008.

15. N. Cornet, G. Gebel, A. de Geyer, Journal De Physique Iv, 8 (1998) 63.

16. F. Meier, G. Eigenberger, Electrochim. Acta, 49 (2004) 1731.

17. J. T. Hinatsu, M. Mizuhata, H. Takenaka, J. Electrochem. Soc., 141 (1994) 1493.

18. J. P. Meyers, J. Newman, J. Electrochem. Soc., 149 (2002) A710.

19. T. A. Zawodzinski, C. Derouin, S. Radzinski, R. J. Sherman, V. T. Smith, T. E. Springer, S. Gottesfeld, J. Electrochem. Soc., 140 (1993) 1041.

20. T. A. Zawodzinski, T. E. Springer, J. Davey, R. Jestel, C. Lopez, J. Valerio, S. Gottesfeld, J. Electrochem. Soc., 140 (1993) 1981. 
21. P. Schröder, Z. Phys. Chem., 45 (1903) 75.

22. K. A. Mauritz, R. B. Moore, Chem. Rev., 104 (2004) 4535.

23. M. Falk, Can. J. Chem., 58 (1980) 1495.

24. W. Y. Hsu, T. D. Gierke, J. Membr. Sci., 13 (1983) 307.

25. W. Y. Hsu, J. R. Barkley, P. Meakin, Macromolecules, 13 (1980) 198.

26. F. P. Orfino, S. Holdcroft, J. New Mater. Electrochem. Syst., 3 (2000) 285.

27. A. Vishnyakov, A. V. Neimark, J. Phys. Chem. B, 105 (2001) 9586.

28. E. Spohr, P. Commer, A. A. Kornyshev, J. Phys. Chem. B, 106 (2002) 10560.

29. G. Gebel, P. Aldebert, M. Pineri, Polymer, 34 (1993) 333.

30. R. S. McLean, M. Doyle, B. B. Sauer, Macromolecules, 33 (2000) 6541.

31. P. Choi, R. Datta, J. Electrochem. Soc., 150 (2003) E601.

32. J. Divisek, M. Eikerling, V. Mazin, H. Schmitz, U. Stimming, Y. M. Volfkovich, J. Electrochem. Soc., 145 (1998) 2677.

33. K. D. Kreuer, J. Membr. Sci., 185 (2001) 29.

34. V. Gurau, F. Barbir, H. Liu, J. Electrochem. Soc., 147 (2000) 2468.

35. T. E. Springer, M. S. Wilson, S. Gottesfeld, J. Electrochem. Soc., 140 (1993) 3513.

36. A. A. Kulikovsky, Fuel Cells, 1 (2001) 162.

37. D. Bevers, M. Wohr, K. Yasuda, K. Oguro, J. Appl. Electrochem., 27 (1997) 1254.

38. W. He, J. S. Yi, T. V. Nguyen, AIChE J., 46 (2000) 2053.

39. D. Natarajan, T. V. Nguyen, J. Power Sources, 115 (2003) 66.

40. D. Natarajan, T. V. Nguyen, J. Electrochem. Soc., 148 (2001) A1324.

41. J. S. Yi, T. V. Nguyen, J. Electrochem. Soc., 146 (1999) 38.

42. Z. H. Wang, C. Y. Wang, K. S. Chen, J. Power Sources, 94 (2001) 40.

43. J. H. Nam, M. Kaviany, Int. J. Heat Mass Transfer, 46 (2003) 4595. 
44. T. Berning, N. Djilali, J. Electrochem. Soc., 150 (2003) A1598.

45. S. J. Ridge, R. E. White, Y. Tsou, R. N. Beaver, G. A. Eisman, J. Electrochem. Soc., 136 (1989) 1902.

46. S. Mazumder, J. V. Cole, J. Electrochem. Soc., 150 (2003) A1510.

47. E. Birgersson, M. Noponen, M. Vynnycky, J. Electrochem. Soc., 152 (2005) A1021.

48. J. L. Yuan, B. Sunden, M. Hou, H. M. Zhang, Num. Heat Transf. A, 46 (2004) 669.

49. C. Marr, X. G. Li, J. Power Sources, 77 (1999) 17.

50. Y. W. Rho, S. Srinivasan, Y. T. Kho, J. Electrochem. Soc., 141 (1994) 2089.

51. R. F. Mann, J. C. Amphlett, M. A. I. Hooper, H. M. Jensen, B. A. Peppley, P. R. Roberge, J. Power Sources, 86 (2000) 173.

52. H.-S. Chu, C. Yeh, F. Chen, J. Power Sources, 123 (2002) 1.

53. J. Leddy, A. E. Iverson, N. E. Vanderborgh, in: J. R. Selman, H. C. Maru (Ed.), Electrochemical and Thermal Modeling of Battery, Fuel Cell, and Photoenergy Conversion Systems, PV 86-12, The Electrochemical Society Proceeding Series, Pennington, Nj, 1986, pp. 310.

54. J. C. Amphlett, R. M. Baumert, R. F. Mann, B. A. Peppley, P. R. Roberge, T. J. Harris, J. Electrochem. Soc., 142 (1995) 1.

55. S. Mazumder, J. Electrochem. Soc., 152 (2005) A1633.

56. D. T. Song, Q. P. Wang, Z. S. Liu, M. Eikerling, Z. Xie, T. Navessin, S. Holdcroft, Electrochim. Acta, 50 (2005) 3347.

57. W. Sun, B. A. Peppley, K. Karan, Electrochim. Acta, 50 (2005) 3359.

58. $\quad$ L. Wang, H. Liu, J. Power Sources, 134 (2004) 185.

59. J. Newman, K. E. Thomas-Alyea, Electrochemical Systems, 3rd ed., John Wiley \& Sons, New York 2004.

60. A. Einstein, Ann. Physik., 17 (1905) 549.

61. W. Nernst, Z. Phys. Chem., 2 (1888) 613.

62. S. Dutta, S. Shimpalee, J. W. Van Zee, J. Appl. Electrochem., 30 (2000) 135. 
63. S. Dutta, S. Shimpalee, J. W. Van Zee, Int. J. Heat Mass Transfer, 44 (2001) 2029.

64. S. Shimpalee, S. Greenway, D. Spuckler, J. W. Van Zee, J. Power Sources, 135 (2004) 79.

65. W. K. Lee, S. Shimpalee, J. W. Van Zee, J. Electrochem. Soc., 150 (2003) A341.

66. T. V. Nguyen, R. E. White, J. Electrochem. Soc., 140 (1993) 2178.

67. J. S. Yi, T. V. Nguyen, J. Electrochem. Soc., 145 (1998) 1149.

68. T. Okada, G. Xie, Y. Tanabe, J. Electroanal. Chem., 413 (1996) 49.

69. T. Okada, G. Xie, M. Meeg, Electrochim. Acta, 43 (1998) 2141.

70. J.-T. Wang, R. F. Savinell, Electrochim. Acta, 37 (1992) 2737.

71. N. P. Siegel, M. W. Ellis, D. J. Nelson, M. R. von Spakovsky, J. Power Sources, 115 (2003) 81 .

72. H. van Bussel, F. G. H. Koene, R. Mallant, J. Power Sources, 71 (1998) 218.

73. S. Shimpalee, S. Dutta, Num. Heat Transf. A, 38 (2000) 111.

74. A. A. Kulikovsky, J. Electrochem. Soc., 150 (2003) A1432.

75. D. R. Sena, E. A. Ticianelli, V. A. Paganin, E. R. Gonzalez, J. Electroanal. Chem., 477 (1999) 164.

76. K. Dannenberg, P. Ekdunge, G. Lindbergh, J. Appl. Electrochem., 30 (2000) 1377.

77. H. Meng, C. Y. Wang, J. Electrochem. Soc., 151 (2004) A358.

78. H. Meng, C. Y. Wang, J. Electrochem. Soc., 152 (2005) A1733.

79. H. Ju, H. Meng, C. Y. Wang, Int. J. Heat Mass Transfer, 48 (2005) 1303.

80. U. Pasaogullari, C. Y. Wang, J. Electrochem. Soc., 152 (2005) A380.

81. U. Pasaogullari, C. Y. Wang, K. S. Chen, J. Electrochem. Soc., 152 (2005) A1574.

82. P. Berg, K. Promislow, J. St Pierre, J. Stumper, B. Wetton, J. Electrochem. Soc., 151 (2004) A341. 
83. D. N. Bennion, Mass Transport of Binary Electrolyte Solutions in Membranes, Water Resources Center Desalination Report No. 4. 1966, Department of Engineering, University of California: Los Angeles, CA.

84. P. N. Pintauro, D. N. Bennion, Ind. Eng. Chem. Fundam., 23 (1984) 230.

85. J. O. Hirschfelder, C. F. Curtiss, R. B. Bird, Molecular Theory of Gases and Liquids, John Wiley \& Sons, Inc., New York 1954.

86. J. Fimrite, B. Carnes, H. Struchtrup, N. Djilali, J. Electrochem. Soc., 152 (2005) A1815.

87. E. A. Mason, A. P. Malinauskas, Gas Transport in Porous Media: The Dusty-Gas Model, Elsevier, Amsterdam 1983.

88. T. Thampan, S. Malhotra, H. Tang, R. Datta, J. Electrochem. Soc., 147 (2000) 3242.

89. M. Wohr, K. Bolwin, W. Schnurnberger, M. Fischer, W. Neubrand, G. Eigenberger, Int. J. Hydrog. Energy 23 (1998) 213.

90. T. F. Fuller, Ph.D. Dissertation, University of California, Berkeley, CA, 1992.

91. A. Z. Weber, J. Newman, J. Electrochem. Soc., 151 (2004) A311.

92. B. Auclair, V. Nikonenko, C. Larchet, M. Metayer, L. Dammak, J. Membr. Sci., 195 (2002) 89.

93. S. Koter, C. H. Hamann, J. Non-Equilib. Therm., 15 (1990) 315.

94. P. Futerko, I. M. Hsing, Electrochim. Acta, 45 (2000) 1741.

95. I. M. Hsing, P. Futerko, Chem. Eng. Sci., 55 (2000) 4209.

96. A. C. West, T. F. Fuller, J. Appl. Electrochem., 26 (1996) 557.

97. G. J. M. Janssen, J. Electrochem. Soc., 148 (2001) A1313.

98. J. P. Meyers, Ph.D. Dissertation, University of California, Berkeley, 1998.

99. M. W. Verbrugge, R. F. Hill, J. Electrochem. Soc., 137 (1990) 1131.

100. M. W. Verbrugge, R. F. Hill, J. Electrochem. Soc., 137 (1990) 886.

101. R. Schlögl, Z. Phys. Chem., 3 (1955) 73.

102. V. Gurau, H. Liu, S. Kakac, AIChE J., 44 (1998) 2414. 
103. L. Pisani, G. Murgia, M. Valentini, B. D'Aguanno, J. Electrochem. Soc., 149 (2002) A898.

104. G. Murgia, L. Pisani, M. Valentini, B. D'Aguanno, J. Electrochem. Soc., 149 (2002) A31.

105. T. Berning, D. M. Lu, N. Djilali, J. Power Sources, 106 (2002) 284.

106. D. Singh, D. M. Lu, N. Djilali, Int. J. Eng. Sci., 37 (1999) 431.

107. J. J. Baschuk, X. Li, J. Power Sources, 86 (2000) 181.

108. S. Um, C.-Y. Wang, K. S. Chen, J. Electrochem. Soc., 147 (2000) 4485.

109. B. R. Sivertsen, N. Djilali, J. Power Sources, 141 (2005) 65.

110. G. J. M. Janssen, M. L. J. Overvelde, J. Power Sources, 101 (2001) 117.

111. A. Rowe, X. G. Li, J. Power Sources, 102 (2001) 82.

112. P. Costamagna, Chem. Eng. Sci., 56 (2001) 323.

113. K. Hertwig, L. Martens, R. Karwoth, Fuel Cells, 2 (2002) 61.

114. L. X. You, H. T. Liu, Int. J. Heat Mass Transfer, 45 (2002) 2277.

115. P. W. Li, L. Schaefer, Q. M. Wang, T. Zhang, M. K. Chyu, J. Power Sources, 115 (2003) 90.

116. M. De Francesco, E. Arato, P. Costa, J. Power Sources, 132 (2004) 127.

117. A. Z. Weber, J. Newman, J. Electrochem. Soc., 152 (2005) A677.

118. A. Z. Weber, J. Newman, J. Electrochem. Soc., 151 (2004) A326.

119. A. Z. Weber, R. M. Darling, J. Newman, J. Electrochem. Soc., 151 (2004) A1715.

120. C. Ziegler, H. M. Yu, J. O. Schumacher, J. Electrochem. Soc., 152 (2005) A1555.

121. A. A. Kulikovsky, T. Wuster, A. Egmen, D. Stolten, J. Electrochem. Soc., 152 (2005) A1290.

122. P. Futerko, I. M. Hsing, J. Electrochem. Soc., 146 (1999) 2049.

123. Y. Y. Shan, S. Y. Choe, J. Power Sources, 145 (2005) 30.

124. S. Mazumder, J. V. Cole, J. Electrochem. Soc., 150 (2003) A1503. 
125. J. Ramousse, J. Deseure, O. Lottin, S. Didierjean, D. Maillet, J. Power Sources, 145 (2005) 416.

126. R. B. Bird, W. E. Stewart, E. N. Lightfoot, Transport Phenomena, 2nd ed., John Wiley \& Sons, Inc., New York 2002.

127. J. Newman, Ind. Eng. Chem. Res., 34 (1995) 3208.

128. L. Rao, J. Newman, J. Electrochem. Soc., 144 (1997) 2697.

129. K. E. Thomas, J. Newman, J. Electrochem. Soc., 150 (2003) A176.

130. J. Xie, D. L. Wood, D. M. Wayne, T. A. Zawodzinski, P. Atanassov, R. L. Borup, J. Electrochem. Soc., 152 (2005) A104.

131. V. Stamenkovic, B. N. Grgur, P. N. Ross, N. M. Markovic, J. Electrochem. Soc., 152 (2005) A277.

132. D. E. Curtin, R. D. Lousenberg, T. J. Henry, P. C. Tangeman, M. E. Tisack, J. Power Sources, 131 (2004) 41.

133. N. P. Siegel, M. W. Ellis, D. J. Nelson, M. R. von Spakovsky, J. Power Sources, 128 (2004) 173.

134. T. Zhou, H. Liu, Int. J. Trans. Phen., 3 (2001) 177.

135. M. Eikerling, Y. I. Kharkats, A. A. Kornyshev, Y. M. Volfkovich, J. Electrochem. Soc., 145 (1998) 2684.

136. M. Eikerling, A. A. Kornyshev, U. Stimming, J. Phys. Chem. B, 101 (1997) 10807.

137. R. H. Perry, D. W. Green, Perry's Chemical Engineers' Handbook, 7th ed., J. O. Maloney, Editor, McGraw-Hill, New York 1997.

138. T. Sakai, H. Takenaka, N. Wakabayashi, Y. Kawami, E. Torikai, J. Electrochem. Soc., $132(1985) 1328$.

139. D. M. Bernardi, J. Electrochem. Soc., 137 (1990) 3344.

140. R. Bradean, K. Promislow, B. Wetton, Num. Heat Transf. A, 42 (2002) 121.

141. S. Patankar, Numerical Heat Transfer and Fluid Flow, Hemisphere Publishing Corporation 1980.

142. A. A. Kulikovsky, Electrochem. Commun., 4 (2002) 527. 
143. T. Okada, J. Electroanal. Chem., 465 (1999) 1.

144. F. L. Chen, Y. G. Su, C. Y. Soong, W. M. Yan, H. S. Chu, J. Electroanal. Chem., 566 (2004) 85 .

145. W. M. Yan, F. L. Chen, H. Y. Wu, C. Y. Soong, H. S. Chu, J. Power Sources, 129 (2004) 127.

146. D. R. Morris, X. D. Sun, J. Appl. Polym. Sci., 50 (1993) 1445.

147. K. Broka, P. Ekdunge, J. Appl. Electrochem., 27 (1997) 117.

148. C. M. Gates, J. Newman, AIChE J., 46 (2000) 2076.

149. S. H. Ge, X. G. Li, B. L. Yi, I. M. Hsing, J. Electrochem. Soc., 152 (2005) A1149.

150. T. V. Nguyen, N. Vanderborgh, J. Membr. Sci., 143 (1998) 235.

151. V. K. Datye, P. L. Taylor, A. J. Hopfinger, Macromolecules, 17 (1984) 1704.

152. B. Dreyfus, J. Polym. Sci., Part B: Polym. Phys., 21 (1983) 2337.

153. K. A. Mauritz, C. E. Rogers, Macromolecules, 18 (1985) 483.

154. P. Choi, N. H. Jalani, R. Datta, J. Electrochem. Soc., 152 (2005) E84.

155. C. Tsonos, L. Apekis, P. Pissis, J. Mater. Sci., 35 (2000) 5957.

156. M. Eikerling, A. A. Kornyshev, A. M. Kuznetsov, J. Ulstrup, S. Walbran, J. Phys. Chem. B, 105 (2001) 3646.

157. J. M. Prausnitz, R. N. Lichtenthaler, E. G. Azevedo, Molecular Thermodynamics of Fluid-Phase Equilibria, 3rd Ed., Prentice-Hall, Inc., Upper Saddle River, New Jersey 1999.

158. T. J. Edwards, M.S. Thesis, University of California, Berkeley, 1974.

159. V. Freger, Polymer, 43 (2002) 71.

160. F. A. L. Dullien, Porous Media: Fluid Transport and Pore Structure, 2nd ed., Academic Press, Inc., New York 1992.

161. A. Z. Weber, J. Newman, AIChE J., 50 (2004) 3215.

162. J. Bear, Dynamics of Fluids in Porous Media, Dover Publications, Inc., New York 1988. 
163. M. W. Verbrugge, R. F. Hill, J. Phys. Chem., 92 (1988) 6778.

164. Y. Wang, C. Y. Wang, J. Electrochem. Soc., 152 (2005) A445.

165. S. Um, C. Y. Wang, J. Power Sources, 125 (2004) 40.

166. K. R. Weisbrod, S. A. Grot, N. E. Vanderborgh, in: A. R. Landgrebe, S. Gottesfeld, G. Halpert (Ed.), First International Symposium on Proton Conducting Membrane Fuel Cells, PV 95-23, The Electrochemical Society Proceeding Series, Pennington, NJ, 1995, pp. 153.

167. P. N. Pintauro, D. N. Bennion, Ind. Eng. Chem. Fundam., 23 (1984) 234.

168. G. Gebel, Polymer, 41 (2000) 5829.

169. C. H. Hsu, C. C. Wan, J. Power Sources, 115 (2003) 268.

170. R. S. Yeo, C. H. Cheng, J. Appl. Polym. Sci., 32 (1986) 5733.

171. J. A. Elliott, S. Hanna, A. M. S. Elliott, G. E. Cooley, Polymer, 42 (2001) 2251.

172. A. Z. Weber, Ph.D. Dissertation, University of California, Berkeley, 2004.

173. G. Gebel, P. Aldebert, M. Pineri, Macromolecules, 20 (1997) 1425.

174. J. P. Meyers, J. Newman, J. Electrochem. Soc., 149 (2002) A718.

175. S. Slade, S. A. Campbell, T. R. Ralph, F. C. Walsh, J. Electrochem. Soc., 149 (2002) A1556.

176. P. Choi, N. H. Jalani, R. Datta, J. Electrochem. Soc., 152 (2005) E123.

177. N. Agmon, Chem. Phys. Lett., 244 (1995) 456.

178. B. R. Breslau, I. F. Miller, Ind. Eng. Chem. Fundam., 10 (1971) 554.

179. G. Karimi, X. Li, J. Power Sources, 140 (2005) 1.

180. S. Koter, J. Membr. Sci., 166 (2000) 127.

181. A. Narebska, S. Koter, Electrochim. Acta, 32 (1987) 449.

182. S. Motupally, A. J. Becker, J. W. Weidner, J. Electrochem. Soc., 147 (2000) 3171. 
183. T. A. Zawodzinski, M. Neeman, L. O. Sillerud, S. Gottesfeld, J. Phys. Chem., 95 (1991) 6040 .

184. R. E. De La Rue, C. W. Tobias, J. Electrochem. Soc., 106 (1959) 827.

185. D. A. G. Bruggeman, Ann. Physik., 24 (1935) 636.

186. G. Li, P. P. Pickup, J. Electrochem. Soc., 150 (2003) C745.

187. D. M. Bernardi, Ph.D. Dissertation, University of California, Berkeley, 1986.

188. D. M. Bernardi, E. Pawlikowski, J. Newman, J. Electrochem. Soc., 132 (1985) 5.

189. J. N. Agar, in: P. Delahay (Ed.), Advances in Electrochemistry and Electrochemical Engineering, 3, John Wiley \& Sons, New York, 1963, pp. 32.

190. H. C. Ju, C. Y. Wang, S. Cleghorn, U. Beuscher, J. Electrochem. Soc., 152 (2005) A1645.

191. A. Z. Weber, J. Newman, in: J. W. Van Zee, T. F. Fuller, S. Gottesfeld, M. Murthy (Ed.), Proton Conducting Membrane Fuel Cells Iv, The Electrochemical Society Proceeding Series, Pennington, NJ, 2004,

192. M. Eikerling, A. A. Kornyshev, J. Electroanal. Chem., 453 (1998) 89.

193. L. X. You, H. T. Liu, Int. J. Hydrog. Energy 26 (2001) 991.

194. L. Pisani, M. Valentini, G. Murgia, J. Electrochem. Soc., 150 (2003) A1558.

195. M. L. Perry, J. Newman, E. J. Cairns, J. Electrochem. Soc., 145 (1998) 5.

196. M. Maja, P. Tosco, M. Vanni, J. Electrochem. Soc., 148 (2003) A1368.

197. F. Jaouen, G. Lindbergh, G. Sundholm, J. Electrochem. Soc., 149 (2002) A437.

198. R. P. Iczkowski, M. B. Cutlip, J. Electrochem. Soc., 127 (1980) 1433.

199. Z. N. Farhat, J. Power Sources, 138 (2004) 68.

200. H. S. Fogler, Elements of Chemical Reaction Engineering, 2nd ed., Prentice-Hall, Upper Saddle River, NJ 1992.

201. E. W. Thiele, Ind. Eng. Chem., 31 (1939) 916.

202. E. A. Ticianelli, C. R. Derouin, A. Redondo, S. Srinivasan, J. Electrochem. Soc., 135 (1988) 2209. 
203. Z. G. Qi, A. Kaufman, J. Power Sources, 109 (2002) 38.

204. F. Gloaguen, P. Convert, S. Gamburzev, O. A. Velev, S. Srinivasan, Electrochim. Acta, 43 (1998) 3767.

205. K. Broka, P. Ekdunge, J. Appl. Electrochem., 27 (1997) 281.

206. S. H. Chan, W. A. Tun, Chem. Eng. Tech., 24 (2001) 51.

207. T. Okada, G. Xie, O. Gorseth, S. Kjelstrup, N. Nakamura, T. Arimura, Electrochim. Acta, 43 (1998) 3741.

208. T. Okada, J. New Mater. Electrochem. Syst., 4 (2001) 209.

209. T. Okada, J. Electroanal. Chem., 465 (1999) 18.

210. T. Okada, N. Nakamura, M. Yuasa, I. Sekine, J. Electrochem. Soc., 142 (1995) 4143.

211. S. W. Capeci, P. N. Pintauro, D. N. Bennion, J. Electrochem. Soc., 136 (1989) 2876.

212. R. Tandon, P. N. Pintauro, J. Membr. Sci., 136 (1997) 207.

213. J. R. Bontha, P. N. Pintauro, Chem. Eng. Sci., 49 (1994) 3835.

214. P. N. Pintauro, M. W. Verbrugge, J. Membr. Sci., 44 (1989) 197.

215. F. J. Booth, J. Chem. Phys., 19 (1951) 391.

216. R. M. Darling, J. P. Meyers, J. Electrochem. Soc., 152 (2005) A242.

217. R. M. Darling, J. P. Meyers, J. Electrochem. Soc., 150 (2003) A1523.

218. M. Schulze, A. Schneider, E. Gulzow, J. Power Sources, 127 (2004) 213.

219. J. Xie, D. L. Wood, K. L. More, P. Atanassov, R. L. Borup, J. Electrochem. Soc., 152 (2005) A1011.

220. S. Koter, J. Membr. Sci., 206 (2002) 201.

221. Y. H. Yang, P. N. Pintauro, AIChE J., 46 (2000) 1177.

222. A. G. Guzman-Garcia, P. N. Pintauro, M. W. Verbrugge, R. F. Hill, AIChE J., 36 (1990) 1061.

223. W. Liu, K. Ruth, G. Rusch, J. New Mater. Electrochem. Syst., 4 (2001) 227. 
224. A. Pozio, R. F. Silva, M. De Francesco, L. Giorgi, Electrochim. Acta, 48 (2003) 1543.

225. J. Kiwi, N. Denisov, Y. Gak, N. Ovanesyan, P. A. Buffat, E. Suvorova, F. Gostev, A. Titov, O. Sarkison, P. Albers, V. Nadtochenko, Langmuir, 18 (2002) 9054.

226. Y. Wang, C. Y. Wang, Electrochim. Acta, 50 (2005) 1307.

227. S. Kim, S. Shimpalee, J. W. Van Zee, J. Power Sources, 135 (2004) 110.

228. M. Pianca, E. Barchiesi, G. Esposto, S. Radice, J. Fluorine Chem., 95 (1999) 71.

229. U. A. Paulus, T. J. Schmidt, H. A. Gasteiger, R. J. Behm, J. Electroanal. Chem., 495 (2001) 134 .

230. K. H. Kangasniemi, D. A. Condit, T. D. Jarvi, J. Electrochem. Soc., 151 (2004) E125.

231. L. M. Roen, C. H. Paik, T. D. Jarvi, Electrochem. Solid-State Lett., 7 (2004) A19.

232. C. A. Reiser, L. Bregoli, T. W. Patterson, J. S. Yi, J. D. L. Yang, M. L. Perry, T. D. Jarvi, Electrochem. Solid-State Lett., 8 (2005) A273.

233. H. Wendt, T. Brenscheidt, A. Fischer, Philosophical Transactions of the Royal Society of London Series a-Mathematical Physical and Engineering Sciences, 354 (1996) 1627.

234. R. Z. Jiang, D. Chu, J. Power Sources, 92 (2001) 193.

235. K. Scott, P. Argyropoulos, K. Sundmacher, J. Electroanal. Chem., 477 (1999) 97.

236. K. Scott, W. M. Taama, P. Argyropoulos, K. Sundmacher, J. Power Sources, 83 (1999) 204.

237. J. R. Fan, G. L. Hu, J. Yao, K. F. Cen, Energy Fuels, 16 (2002) 1591.

238. P. Argyropoulos, K. Scott, W. M. Taama, J. Power Sources, 79 (1999) 169.

239. E. Birgersson, J. Nordlund, H. Ekstrom, M. Vynnycky, G. Lindbergh, J. Electrochem. Soc., 150 (2003) A1368.

240. G. Murgia, L. Pisani, A. K. Shukla, K. Scott, J. Electrochem. Soc., 150 (2003) A1231.

241. A. A. Kulikovsky, J. Electrochem. Soc., 152 (2005) A1121.

242. A. A. Kulikovsky, Electrochem. Commun., 6 (2004) 1259.

243. S. S. Sandhu, R. O. Crowther, J. P. Fellner, Electrochim. Acta, 50 (2005) 3985. 


\section{Captions}

Figure 1. Chemical formula of a perfluorinated sulfonic acid membrane, where $\mathrm{m}$ is between 6 and 13 and $\mathrm{z}$ is 3 for Nafion ${ }^{\circledR}$.

Figure 2. Schematic of the membrane showing the various fluxes through it.

Figure 3. Fuel-cell polarization curve showing the various potential losses.

Figure 4. Water-uptake isotherm at $25^{\circ} \mathrm{C}$ showing the effect of Schröder's paradox.

Figure 5. Membrane structure and interactions as a function of water content, $\lambda$ (moles of water per mole of sulfonic acid sites). The top two schematics represent water interacting with the sulfonic acid sites, where the gray waters are more loosely bound. The bottom two schematics are cross-sectional representations of a saturated-vapor- and liquid-equilibrated membrane, respectively, where the gray area is the fluorocarbon matrix, the black is the polymer side chain, the light gray is the water, and the dotted line is a collapsed channel or bridging sulfonic acid site. (Schematics adapted from references [3] and [14] with permission of The Electrochemical Society, Inc.)

Figure 6. More realistic representation of a liquid-equilibrated membrane. (Figure reproduced from reference [33].) 
Figure 7. Simulation results using a diffusive (one-phase) membrane model showing membrane dehydration: $\lambda$ as a function of membrane position (cathode on the left) for different current densities. (Figure reproduced from reference [10] with permission of The Electrochemical Society, Inc.)

Figure 8. Simulation results using a hydraulic (two-phase) membrane model showing the net water flux per proton flux, $\beta$, as a function of current density and cathode-to-anode pressure difference. (Figure reproduced from reference [12] with permission of The Electrochemical Society, Inc.)

Figure 9. Water-flux profiles showing the contributions to the various fluxes, where the anode is much drier than the cathode. (Figure reproduced from reference [55] with permission of The Electrochemical Society, Inc.)

Figure 10. Membrane water content as a function of position both along the gas channel and through the thickness of the membrane for the case of countercurrent operation, dry feed gases, and $0.4 \mathrm{~A} \mathrm{~cm}^{-2}$. (Figure reproduced from reference [118] with permission of The Electrochemical Society, Inc.)

Figure 11. Temperature profile in the membrane as a function of current density for humidified feeds. (Figure adapted from reference [125].) 
Figure 12. Arrhenius plot of the hydrogen permeation coefficient as a function of temperature for a liquid-equilibrated membrane, a vapor-equilibrated membrane, and a dry membrane [14]. Also plotted are the hydrogen permeation coefficients in water [137] and Teflon ${ }^{\circledR}$ [138].

Figure 13. Comparisons of the three test models, PE (dashed), PEF (dotted), and PEA (solid), to experimental data (squares) from (a) Ticianelli et al. [202], (b) Gloaguen et al. [204], and (c) Qi and Kaufman [203] at $60^{\circ} \mathrm{C}$ and $75^{\circ} \mathrm{C}$.

Figure 14. Anode and cathode effectiveness factors as a function of current density for case (a) from Figure 13 using the PEA model.

Figure 15. Comparison of no contamination (left graph) and $10 \% \mathrm{Na}^{+}$contamination on the cathode side of the membrane (right graph) on dimensionless water content (solid line), net water flux (dot-dash line), and membrane potential drop (dashed line) as a function of current density. (Figure reproduced from reference [209]

Figure 16. Potential (a) and dielectric-constant (b) profiles as a function of pore size. (Figure reproduced from reference [214].)

Figure 17. Evolution of water-content profiles when the cathode inlet changes from dry to fully humidified. (Figure reproduced from reference [226].) 


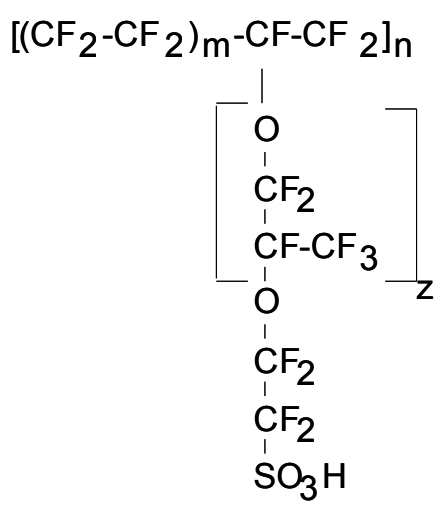

Figure 1. Chemical formula of a perfluorinated sulfonic acid membrane, where $\mathrm{m}$ is between 6 and 13 and $\mathrm{z}$ is 3 for Nafion ${ }^{\circledR}$. 


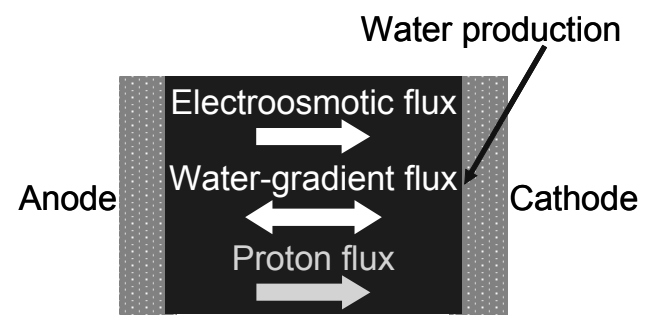

Figure 2. Schematic of the membrane showing the various fluxes through it. 


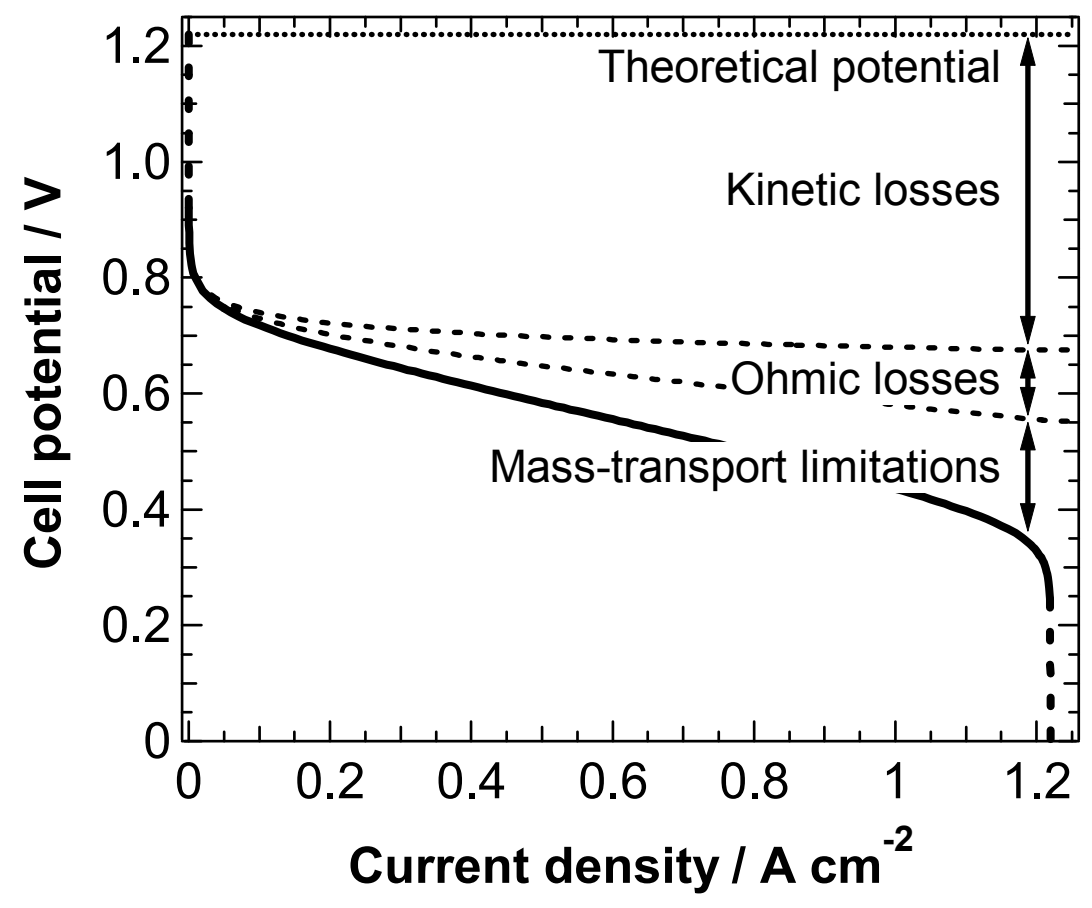

Figure 3. Fuel-cell polarization curve showing the various potential losses. 


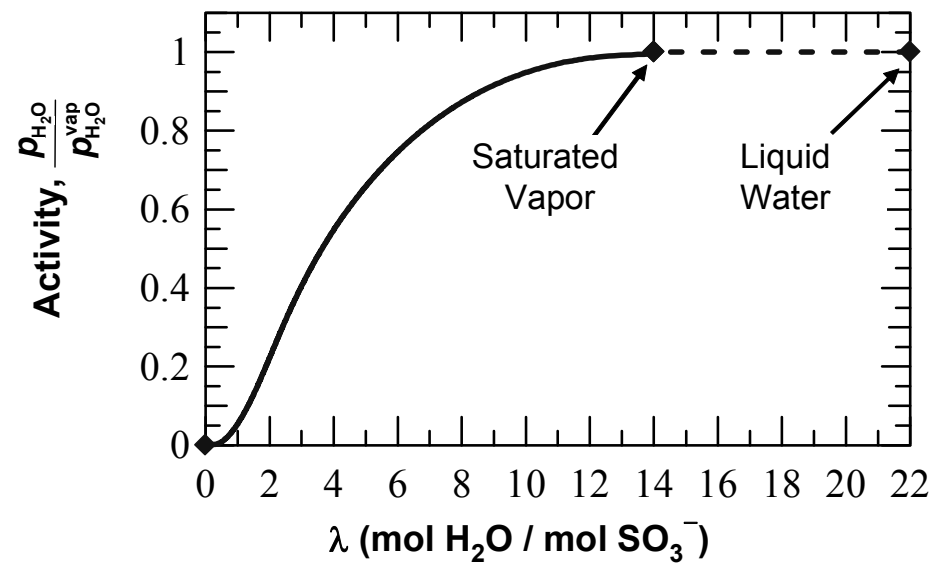

Figure 4. Water-uptake isotherm at $25^{\circ} \mathrm{C}$ showing the effect of Schröder's paradox. 

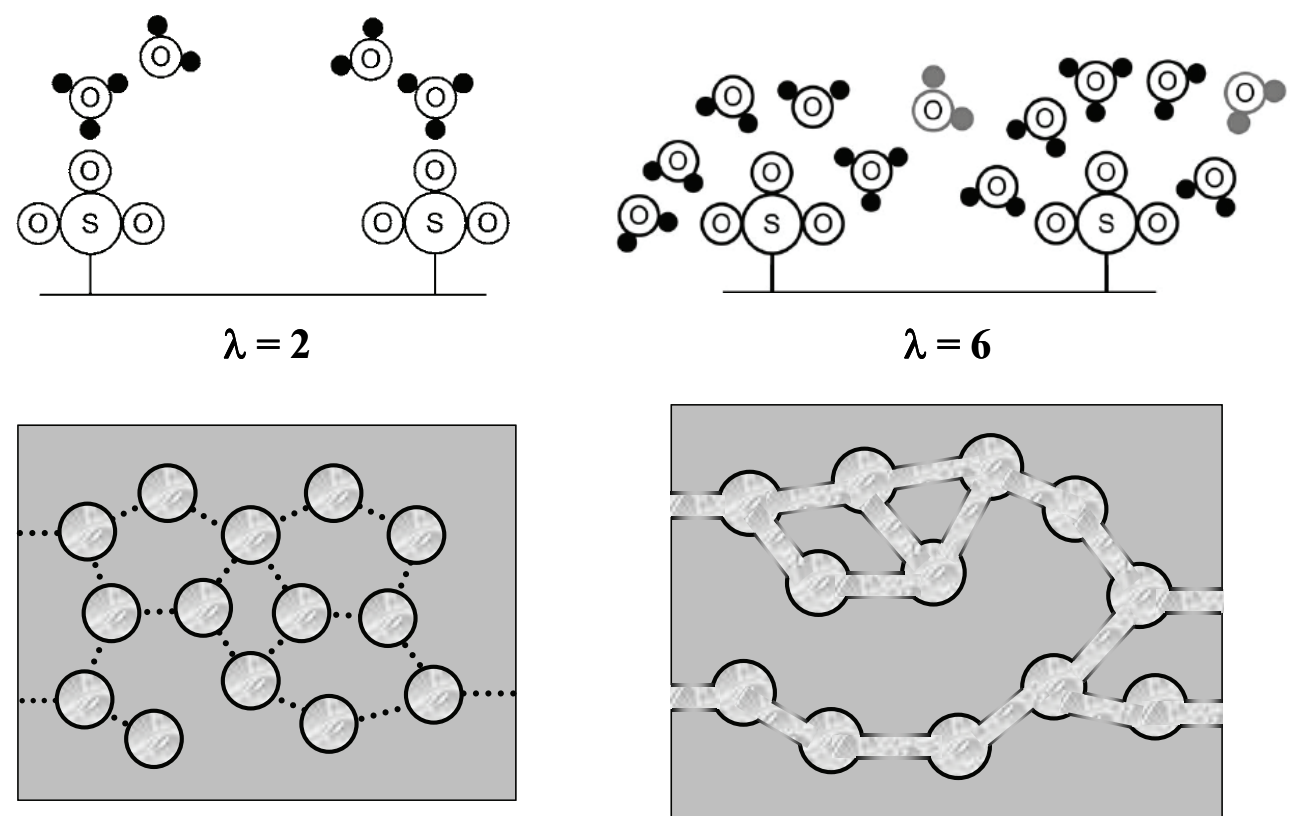

$\lambda=14$

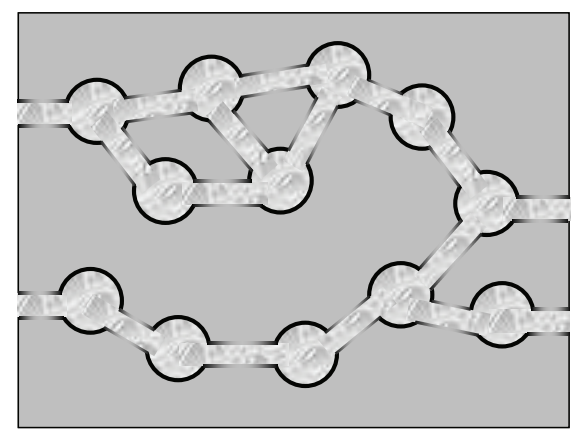

$\lambda=22$

Figure 5. Membrane structure and interactions as a function of water content, $\lambda$ (moles of water per mole of sulfonic acid sites). The top two schematics represent water interacting with the sulfonic acid sites, where the gray waters are more loosely bound. The bottom two schematics are cross-sectional representations of a saturated-vapor- and liquid-equilibrated membrane, respectively, where the gray area is the fluorocarbon matrix, the black is the polymer side chain, the light gray is the water, and the dotted line is a collapsed channel or bridging sulfonic acid site. (Schematics adapted from references [3] and [14] with permission of The Electrochemical Society, Inc.) 


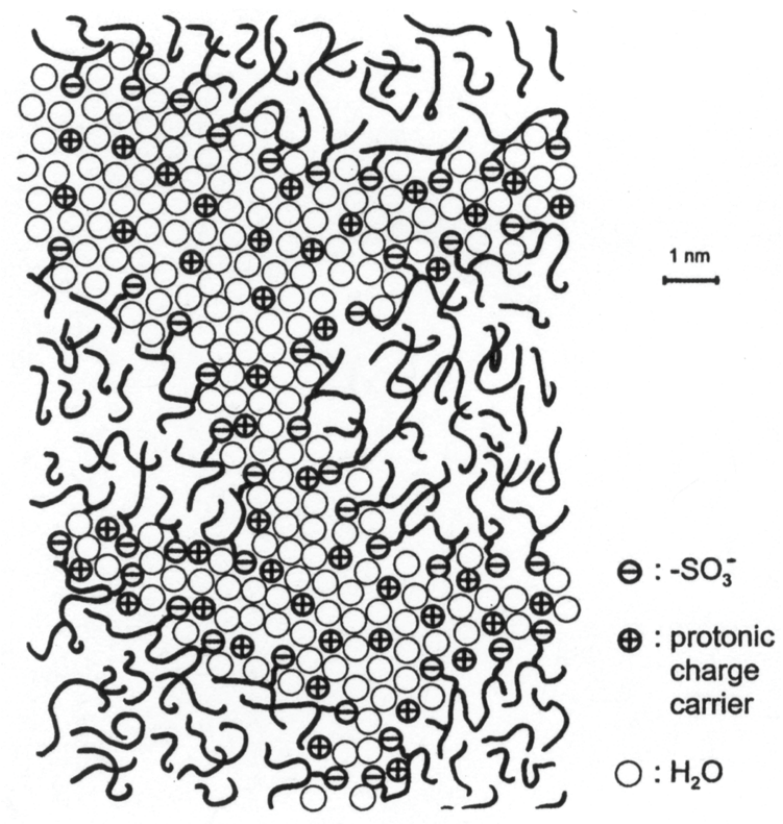

Figure 6. More realistic representation of a liquid-equilibrated membrane. (Figure reproduced from reference [33].) 


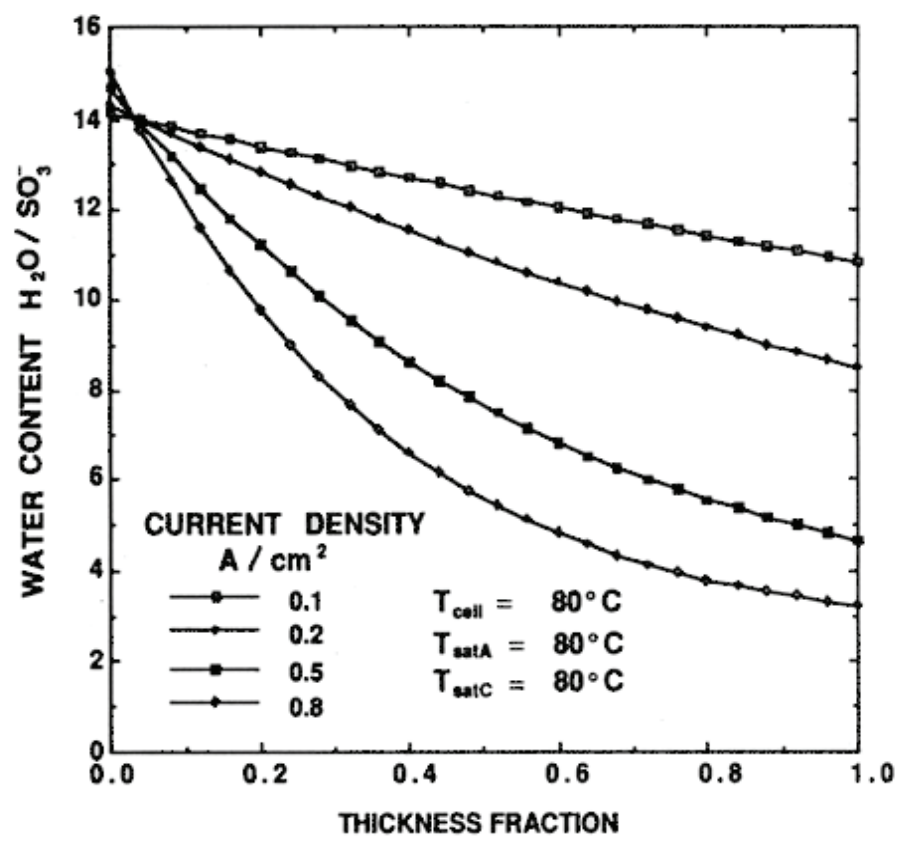

Figure 7. Simulation results using a diffusive (one-phase) membrane model showing membrane dehydration: $\lambda$ as a function of membrane position (cathode on the left) for different current densities. (Figure reproduced from reference [10] with permission of The Electrochemical Society, Inc.) 


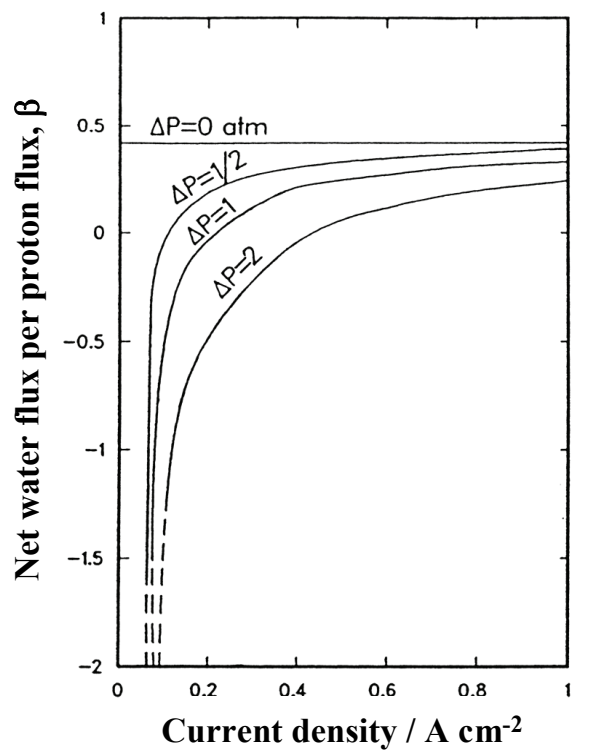

Figure 8. Simulation results using a hydraulic (two-phase) membrane model showing the net water flux per proton flux, $\beta$, as a function of current density and cathode-to-anode pressure difference. (Figure reproduced from reference [12] with permission of The Electrochemical Society, Inc.) 


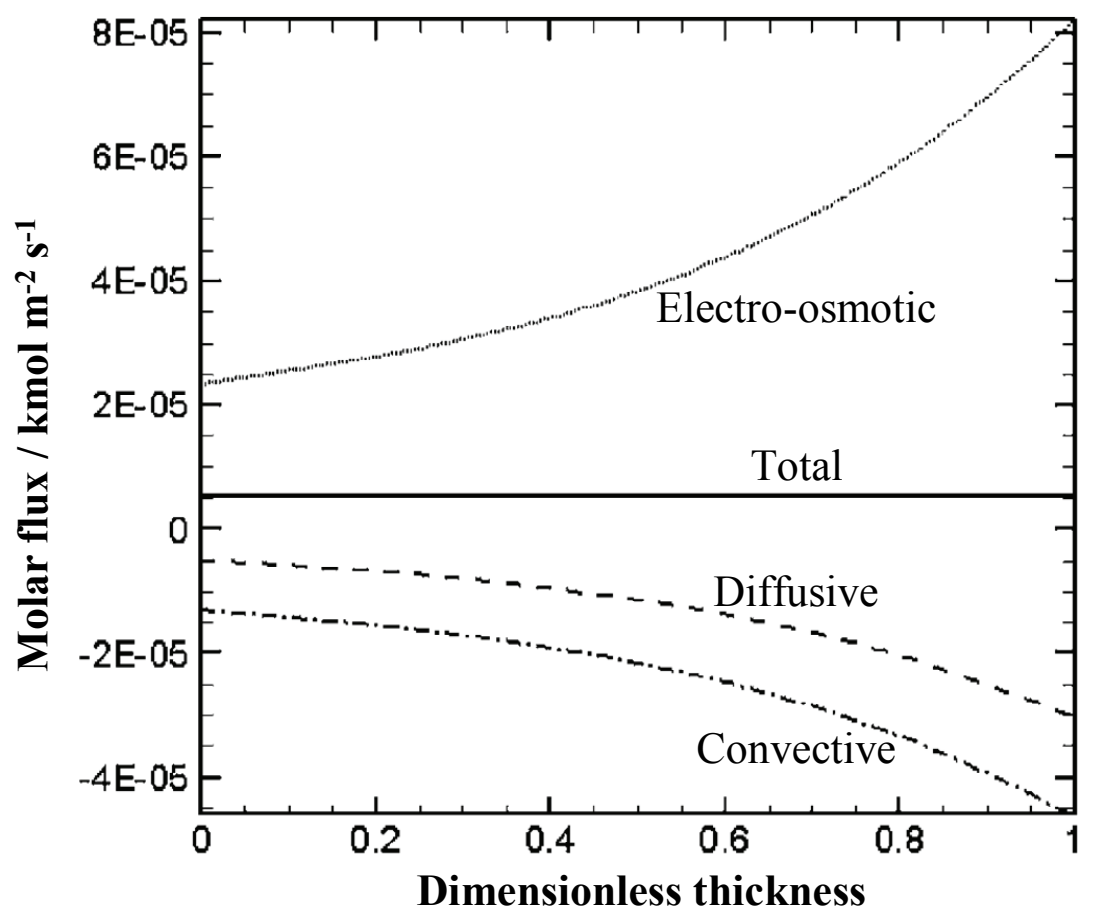

Figure 9. Water-flux profiles showing the contributions to the various fluxes, where the anode is much drier than the cathode. (Figure reproduced from reference [55] with permission of The Electrochemical Society, Inc.) 


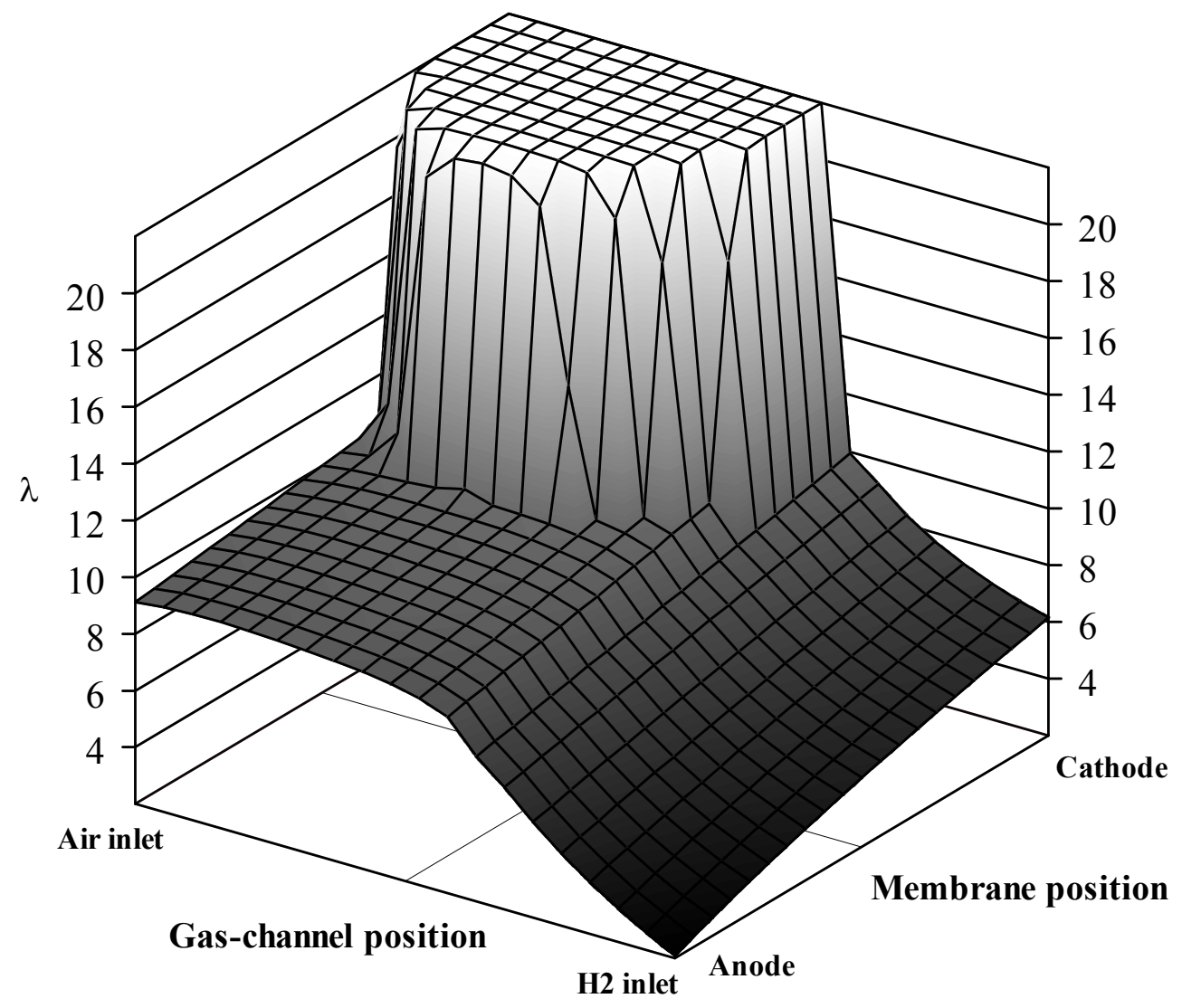

Figure 10. Membrane water content as a function of position both along the gas channel and through the thickness of the membrane for the case of countercurrent operation, dry feed gases, and $0.4 \mathrm{~A} \mathrm{~cm}^{-2}$. (Figure reproduced from reference [118] with permission of The Electrochemical Society, Inc.) 


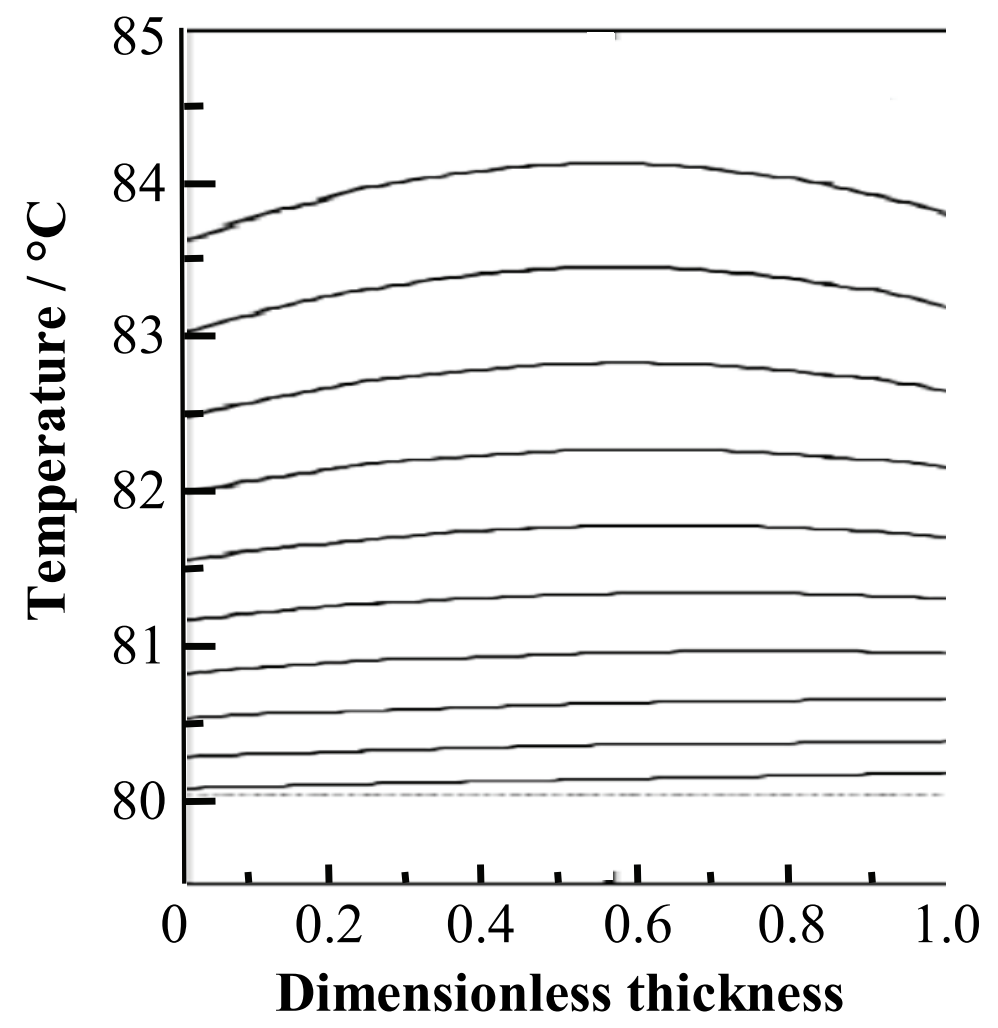

Figure 11. Temperature profile in the membrane as a function of current density for humidified feeds. (Figure adapted from reference [125].) 


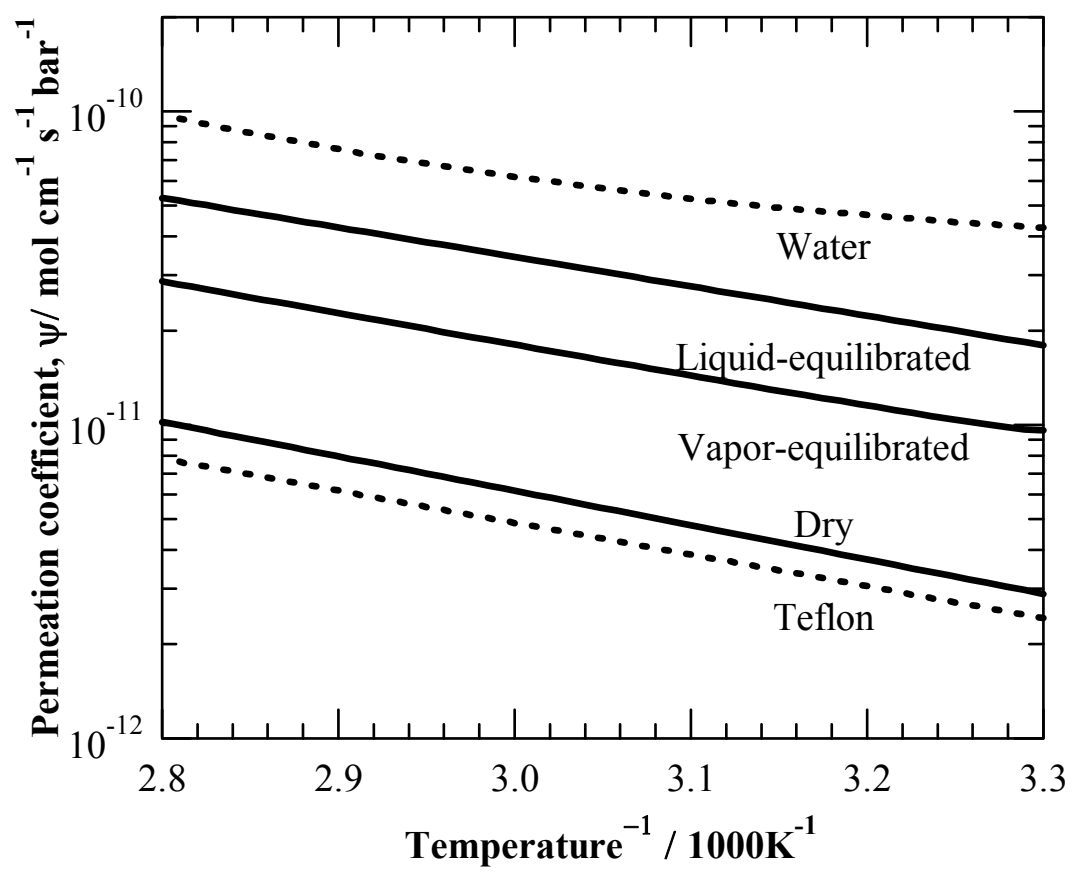

Figure 12. Arrhenius plot of the hydrogen permeation coefficient as a function of temperature for a liquid-equilibrated membrane, a vapor-equilibrated membrane, and a dry membrane [14]. Also plotted are the hydrogen permeation coefficients in water [137] and Teflon ${ }^{\circledR}[138]$. 

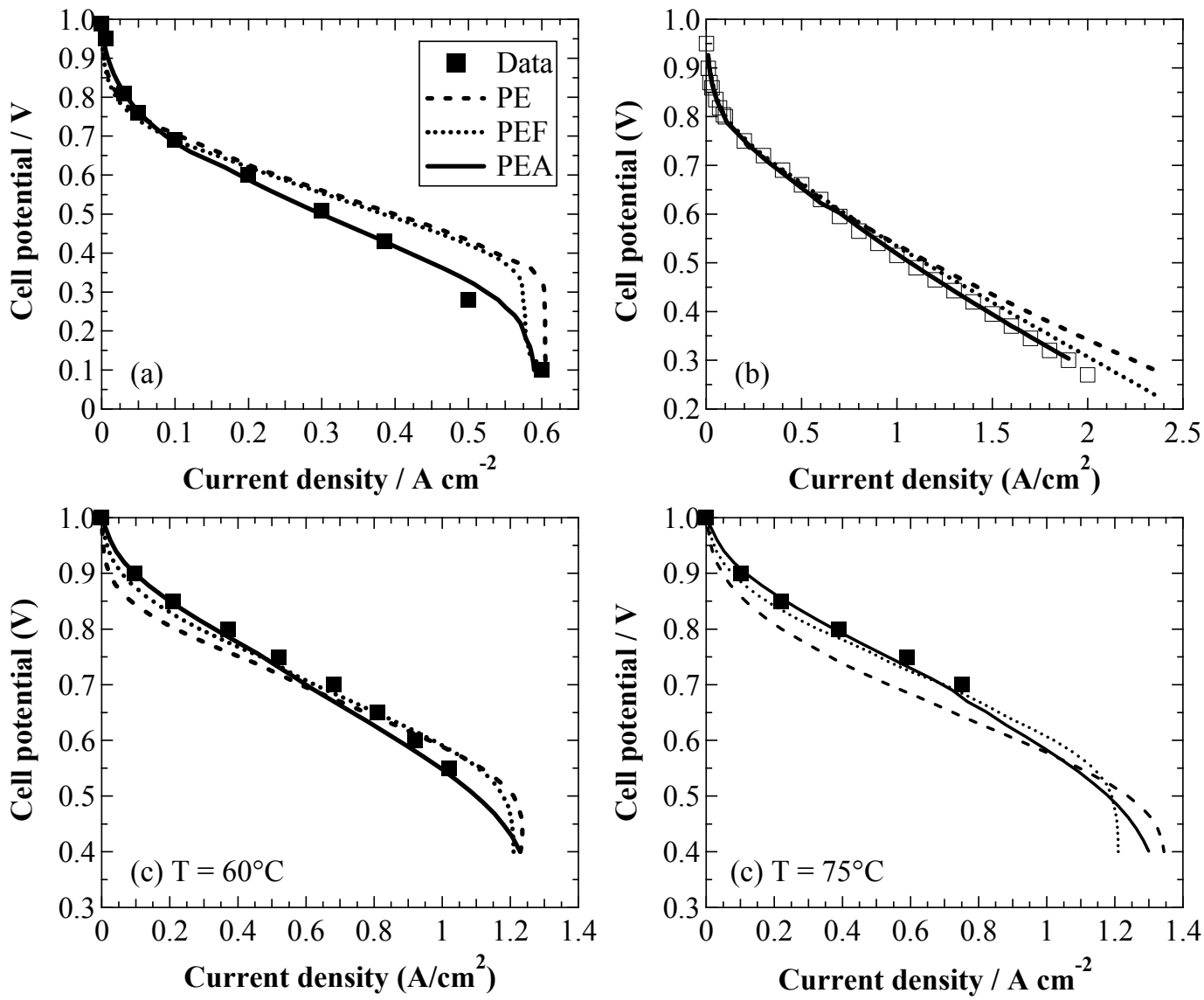

Figure 13. Comparisons of the three test models, PE (dashed), PEF (dotted), and PEA (solid), to experimental data (squares) from (a) Ticianelli et al. [202], (b) Gloaguen et al. [204], and (c) Qi and Kaufman [203] at $60^{\circ} \mathrm{C}$ and $75^{\circ} \mathrm{C}$. 


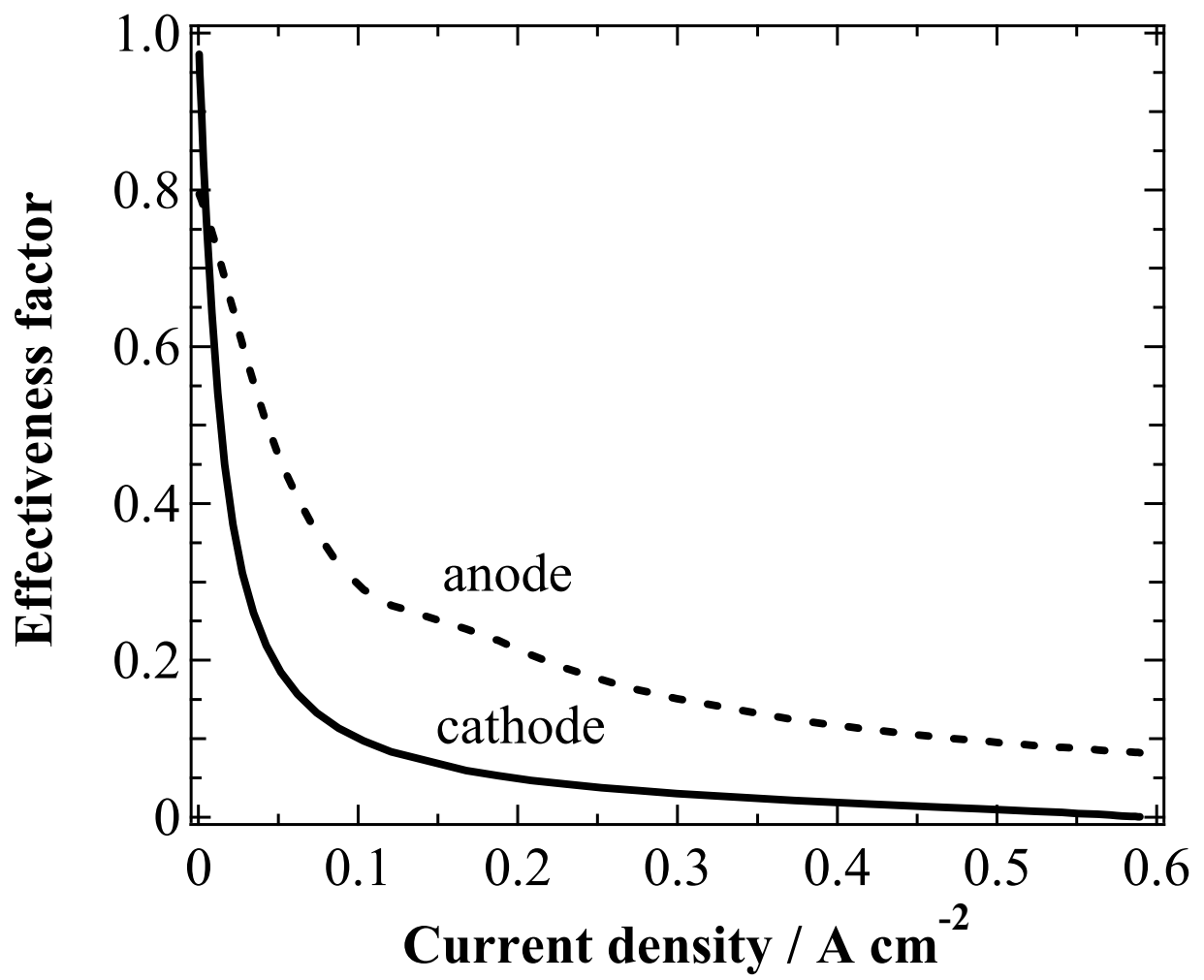

Figure 14. Anode and cathode effectiveness factors as a function of current density for case (a) from Figure 13 using the PEA model. 

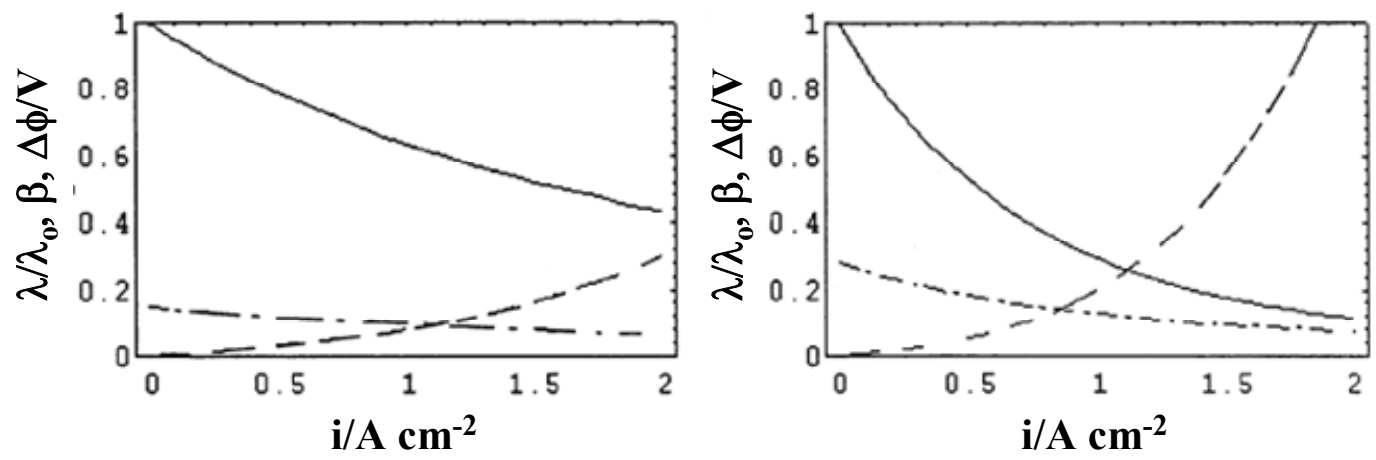

Figure 15. Comparison of no contamination (left graph) and $10 \% \mathrm{Na}^{+}$contamination on the cathode side of the membrane (right graph) on dimensionless water content (solid line), net water flux (dot-dash line), and membrane potential drop (dashed line) as a function of current density. (Figure reproduced from reference [209].) 

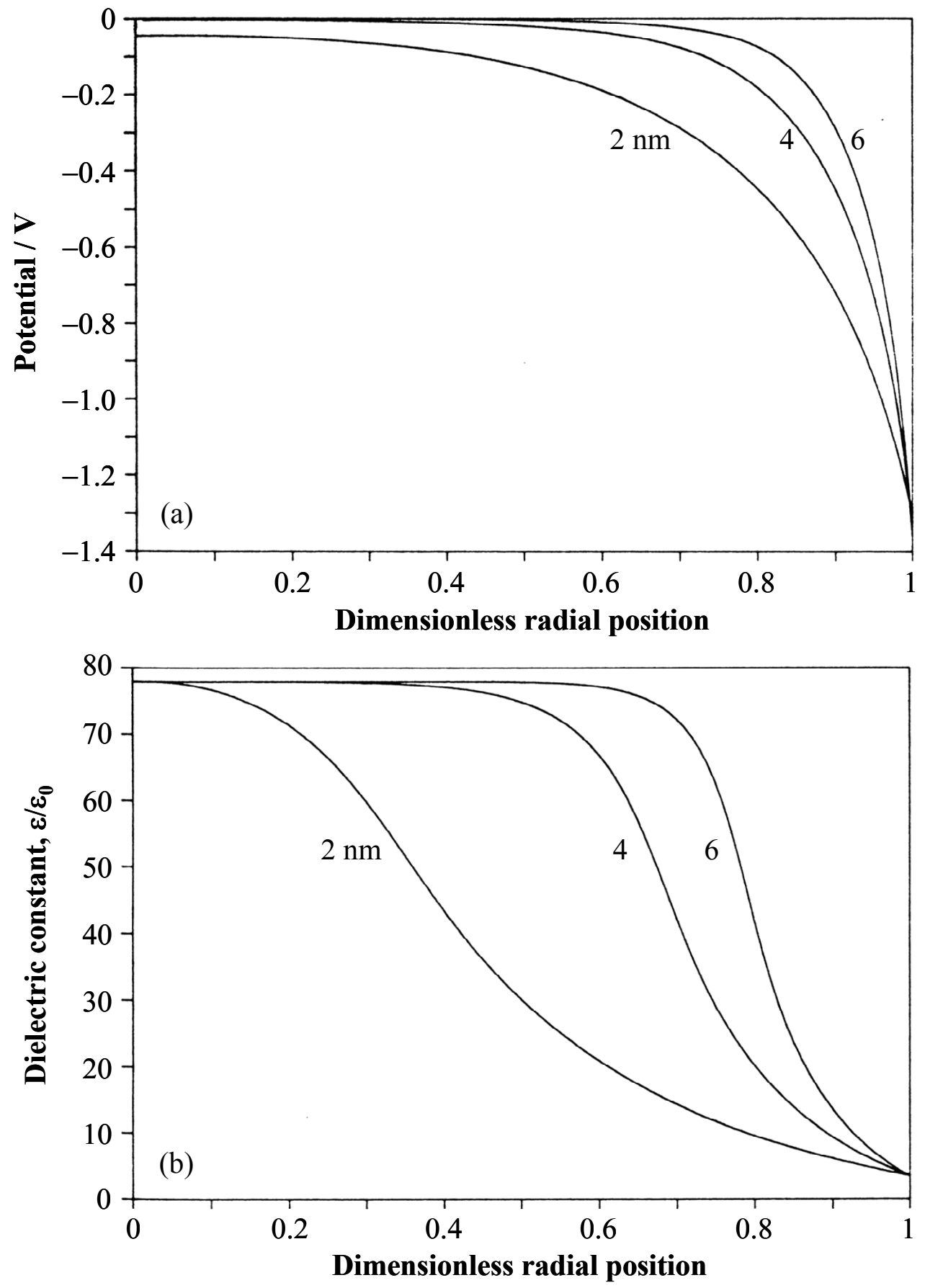

Figure 16. Potential (a) and dielectric-constant (b) profiles as a function of pore size. (Figure reproduced from reference [214].) 


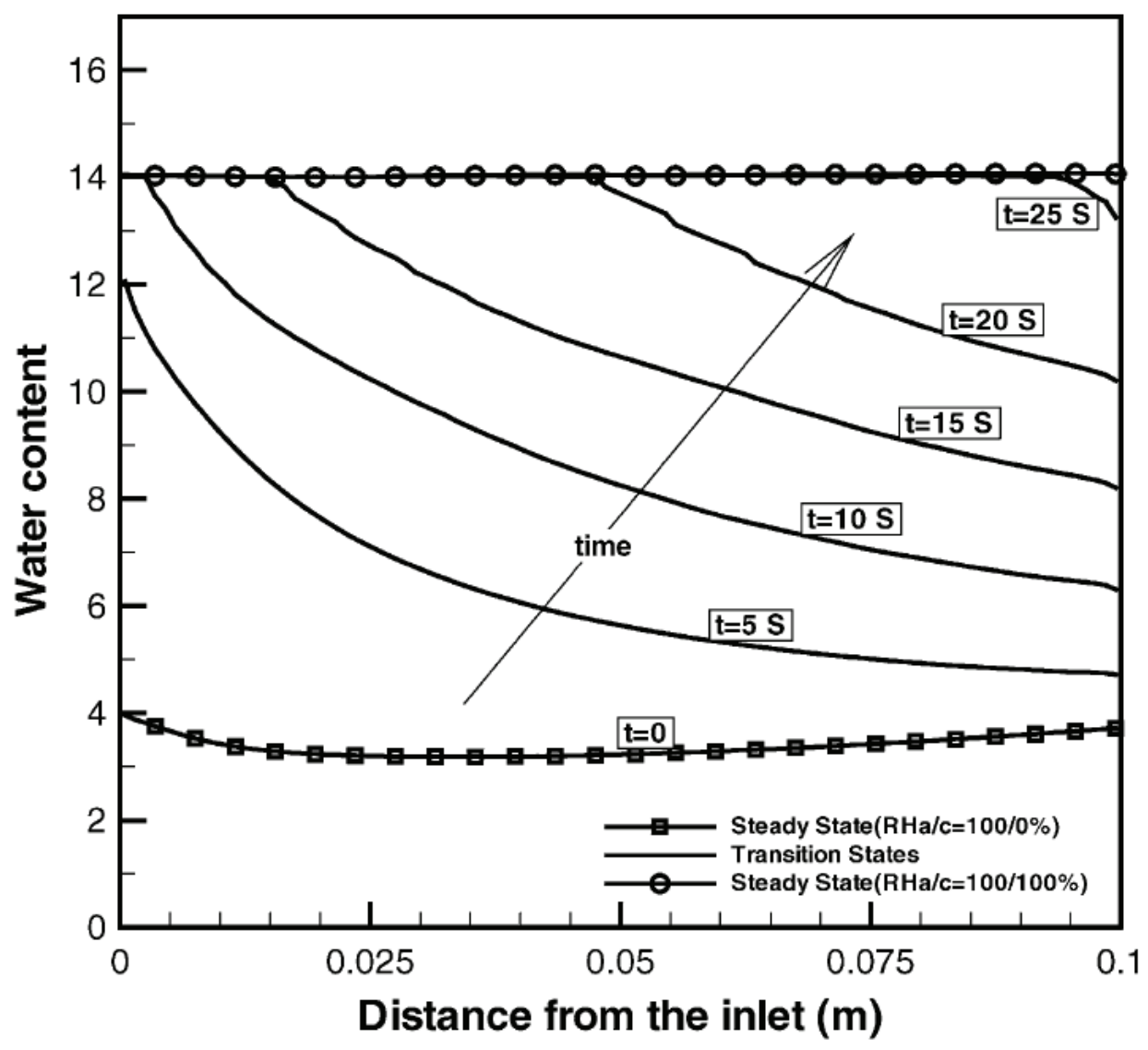

Figure 17. Evolution of water-content profiles when the cathode inlet changes from dry to fully humidified. (Figure reproduced from reference [226].) 